\title{
On the Loewy Series of the Steinberg-PIM of Finite General Linear Groups
}

Von der Fakultät Mathematik und Physik der Universität Stuttgart zur Erlangung der Würde eines Doktors der Naturwissenschaften (Dr. rer. nat.) genehmigte Abhandlung

\section{Vorgelegt von}

\section{Bernd Ackermann}

geboren in Stuttgart

$\begin{array}{ll}\text { Hauptberichter: } & \text { Prof. Dr. R. Dipper } \\ \text { Mitberichter: } & \text { Prof. Dr. M. Geck } \\ & \text { Prof. Dr. W. Kimmerle } \\ \text { Tag der Einreichung: } & 5.7 .2004 \\ \text { Tag der mündlichen Prüfung: } & 19.7 .2004\end{array}$





\section{Contents}

Introduction $\quad$ ii

1 Notation 1

2 Facts about $\mathrm{Gl}_{n}(q)$ and its representations 2

2.1 Combinatorics . . . . . . . . . . . . . . . . 2

2.2 Harish-Chandra induction and restriction . . . . . . . . . . . 2

2.3 Construction of the irreducible modules . . . . . . . . . . . . . . 3

2.4 Harish-Chandra series and block structure . . . . . . . . . . 5

2.5 Steinberg Module and Gelfand-Graev Module . . . . . . . . . 8

3 Steinberg-PIM and Harish-Chandra restriction 9

3.1 The irreducible constituents of the Steinberg-PIM . . . . . . . . 9

3.2 Harish-Chandra restriction of simple modules in the Steinberg-PIM 12

3.3 The special Case $e=1 \ldots \ldots \ldots \ldots$

4 The Loewy series of a PIM $\quad 17$

4.1 General Remarks . . . . . . . . . . . . . . . . . . . 17

4.2 Cyclic Defect Groups . . . . . . . . . . . . . . . . . . . . . . . 19

4.3 The structure of $P_{S t}(L)$ and $\operatorname{End}_{F L}\left(P_{S t}(L)\right) \ldots \ldots 22$

4.4 The Endomorphism Ring of an Induced Module . . . . . . . . . . 23

4.5 The Special Case $e=1 \ldots \ldots$. . . . . . . . . . . . 34

4.6 Main Theorem . . . . . . . . . . . . . . . . . . . 35

4.7 Calculation of the Loewy Series . . . . . . . . . . . . . . . 39

5 Conclusions $\quad 46$

$\begin{array}{ll}\text { A German Summary } & 48\end{array}$

B The Mullineux Map $\quad 51$

$\begin{array}{ll}\text { C Examples } & 52\end{array}$ 


\begin{abstract}
This paper calculates the Loewy series of the projective indecomposable module of the unipotent block contained in the Gelfand-Graev module of the finite general linear group in case of non-describing characteristic and Abelian defect group.
\end{abstract}

\title{
Introduction
}

In the modular representation theory of the general linear group in non-describing characteristic a lot is already known. By the work of Dipper and James we have a construction and labelling of the irreducibles ([Di1],[Di2],[Ja3], [DiJa1]) and by the same authors the construction of the decomposition matrix has been reduced to the construction of the decomposition matrix of the $q$-Schur-algebra [DiJa2]. Also we know the distribution of the irreducibles into Harish-Chandra series [Hi] and into blocks [FoSri2].

In this thesis we want to explore an area where not much is known yet. The projective indecomposable modules form a natural building block of modular representation theory. From general theory we know that for each modular irreducible $D$ there exists a unique projective indecomposable module $P$ such that $D$ is the head (and the socle) of $P$. Also from general theory we know that the decomposition matrix determines the composition factors of $P$. It is a natural question to ask what the inner structure of $P$ looks like, i.e. what the Loewy series of $P$ are.

This question has already been answered for the special case of cyclic defect group by the work of Peacock [Pea] and its specialisation by Fong and Srinivasan [FoSri1]. So we take a look at the more general case of a block with Abelian defect group. Unfortunately, except in special cases we are not able to get a formula for the Loewy series of all projective indecomposables. But we compute the Loewy series of some which are, in some sense, also a building block for the representation theory of general linear groups, namely those contained in the Gelfand-Graev module.

For the purpose of this thesis we restrict ourselves further to the projective indecomposable module which is contained in the unipotent block as well as in the Gelfand-Graev, the projective indecomposable henceforth called the SteinbergPIM. We will give combinatorial formulas for the number of composition factors of each type in each layer of the Loewy series. On the way of proving this we will give two results, which should be interesting in their own right. First of all we will prove that Harish-Chandra restriction of a composition factor of the SteinbergPIM yields a semisimple module. And second we will show that Harish-Chandra restriction of the Steinberg-PIM does respect the layers of the Loewy series. 
In the first chapter crucial notations are established. The second chapter summarises known results on the representation theory of general linear groups which are needed later on. The proofs of these results are largely omitted, but references are given. The third chapter contains the first results. We describe the possible composition factors of the Steinberg-PIM and show that Harish-Chandra restriction is a semisimple functor. In the forth chapter we first describe the special case of cyclic defect and then look at the endomorphism ring of an induced SteinbergPIM of a Levi subgroup. It turns out, that this endomorphism ring contains a sub-algebra, which in relevant cases is isomorphic to a Hecke algebra over the endomorphism ring of said Steinberg-PIM. This in turn allows us the complete treatment of another special case (namely $e=1$ ) where all Loewy series of all PIMs in the unipotent block are given. In the next section we show the fact that Harish-Chandra restriction respects Loewy series. The main ingredients of the proof are that the restriction of semisimple composition factors remains semisimple and that there exists only one composition factor in the Steinberg-PIM which is $e$-regular. We close the chapter with the actual calculation of the Loewy series of the Steinberg-PIM. Since the Loewy series in the case of cyclic defect are completely known we need only some kind of identification procedure to get back from the Loewy series of the Steinberg-PIM of a Levi subgroup to the Loewy series of the Steinberg-PIM of the general linear group. Here the Hecke sub-algebra calculated above is used again. The last chapter gives some closing remarks and outlines some possible routes of further research. We also give a description of the Mullineux map (needed for calculation) and some examples in the appendix.

I wish to thank my supervisor Prof. Dipper for suggesting the area of work and for many helpful discussions. I also thank the Deutsche Forschungsgemeinschaft for their support in the project Di 531/3-1. 



\section{Notation}

We denote by $G:=\mathrm{Gl}_{n}(q)$ the general linear group over the field with $q$ elements, where $q$ is a power of the prime $p$. Let $\ell$ be a prime not dividing $q$ and $e$ be the order of $q$ modulo $\ell$.

Let $(K, O, F)$ be an $\ell$-modular system, where we assume $K$ and $F$ to be algebraically closed. Given any $O G$-lattice $X$, we denote the corresponding ordinary $K G$-module by $X \otimes K G$ or $X_{K}$ and the $\ell$-modular reduction of $X$ by $\bar{X}$. We will write $R$ for any of the rings $\{K, O, F\}$. For any $R$-modules $X$ and $Y$ we write $X \otimes Y$ for the tensor product $X \otimes_{R} Y$.

$G$ is a group with split $(B, N)$-pair (see [Carter], §2) with the standard Borel subgroup $B$ being the group of upper triangular matrices and the group $N$ being generated by the group of diagonal matrices $T$ and the permutation matrices. $T$ is also the maximally split torus in $G$ and the Weyl group $W$ is isomorphic to the symmetric group $S_{n}$.

The root system associated to the group can be taken as $\Phi:=\left\{e_{i}-e_{j} \mid i, j \in\right.$ $\mathbb{N}, 1 \leq i, j \leq n, i \neq j\}$ where $e_{i}$ denotes the standard basis of $\mathbb{R}^{n}$. With the choice of standard Borel subgroup above the simple roots are just $\Delta:=\left\{e_{i}-e_{i+1} \mid 1 \leq\right.$ $i \leq n-1\}$. The positive roots are $\Phi^{+}:=\left\{e_{i}-e_{j} \in \Phi \mid i<j\right\}$. For each $I \subseteq \Delta$ we denote by $\Phi_{I}$ the subset of roots which can be written as $\mathbb{Z}$-linear combinations of roots in $I$.

To each root $\alpha=e_{i}-e_{j} \in \Phi$ we associate a root subgroup of $G$ by $X_{\alpha}:=$ $\left\{1_{n}+x e_{i j} \mid x \in \mathrm{GF}(q)\right\}$, where $\left\{e_{i j}\right\}$ denotes the standard basis of $n \times n$ matrices. We also associate with $\alpha=e_{i}-e_{j}$ an element of the Weyl group $s_{\alpha}:=(i, j) . W$ acts on the roots by permuting the basis elements of $\mathbb{R}^{n}$, so $\alpha s_{\alpha}=-\alpha=e_{j}-e_{i}$. For $I \subseteq \Delta$ we define the group $W_{I}:=<s_{\alpha} \mid \alpha \in I>$ which is a subgroup of $W$. The set $D_{I}:=\left\{w \in W \mid I w \subseteq \Phi^{+}\right\}$, a set of right coset representatives of $W_{I}$ in $W$, is called the set of distinguished coset representatives. For $I, J \subseteq \Delta$ the set $\mathcal{D}_{I, J}:=D_{I} \cap D_{J}^{-1}$ is the set of distinguished double coset representatives of $W_{I} \backslash W / W_{J}$ (see [Carter], §2 for more details).

For $I \subseteq \Delta$ we define some subgroups of $G$ :

$$
\begin{aligned}
& P_{I}:=<T, X_{\alpha} \mid \alpha \in \Phi^{+} \cup \Phi_{I}>\text { standard parabolic subgroup } \\
& L_{I}:=<T, X_{\alpha} \mid \alpha \in \Phi_{I}>\text { standard Levi subgroup } \\
& U_{I}:=<X_{\alpha} \mid \alpha \in \Phi^{+} \backslash \Phi_{I}>\text { the Levi complement }
\end{aligned}
$$

For each $w \in W$ we have $U_{w}^{-}:=\left\langle X_{a} \mid \alpha \in \Phi^{+}, \alpha w \in \Phi^{-}\right\rangle$.

We have that $U_{I}$ is normal in $P_{I}$ and that $P_{I}=L_{I} U_{I}$ (see [Carter], prop 2.6.4). The set of all parabolic subgroups of $G$ is given by $\left\{P_{I}^{g} \mid I \subseteq \Delta, g \in G\right\}$. In 
the same way, the set of all Levi subgroups of $G$ consists of all $G$-conjugates of standard Levi subgroups. We write $\mathcal{L}_{G}$ for the set of standard Levi subgroups of $G$. All modules are right modules unless stated otherwise.

\section{Facts about $\mathrm{Gl}_{n}(q)$ and its representations}

In this section we want to summarise all the facts about the general linear group and its representation theory needed in the rest of the thesis. All theorems and definitions are given only for the general linear group, even if they can be done in a more general setting.

\subsection{Combinatorics}

The representation theory of $G$ is closely related to that of the Hecke algebra $\mathcal{H}_{R}, q\left(S_{n}\right)$ over the symmetric group $S_{n}$ and therefore combinatorics of partitions and tableaux play an important role. We indicate that $\lambda$ is a composition of $n$ by $\lambda \models n$ and by $\lambda \vdash n$ that $\lambda$ is a partition of $n$. The conjugate partition of $\lambda$ is denoted by $\lambda^{\prime}$.

Let $e$ be a positive integer. A partition $\lambda=\left(\lambda_{1}^{k_{1}}, \lambda_{2}^{k_{2}}, \ldots, \lambda_{r}^{k_{r}}\right)$ where $\lambda_{i}>\lambda_{j}$ for $i>j$ is called e-regular, if $k_{i}<e$ for all $i$. $\lambda$ is called $e$-restricted, if $\lambda^{\prime}$ is $e$-regular.

Let $e(d)$ be the least positive integer such that $\ell$ divides $1+q^{d}+q^{2 d}+\cdots+q^{(e(d)-1) d}$. Furthermore let $\tilde{e}(d)$ be the least positive integer such that $\ell$ divides $q^{\tilde{e}(d) d}-1$. Observe that $e=\tilde{e}(1)$ and that $e(d)=\tilde{e}(d)$ if $e(d) \neq \ell$ (or equivalently $e \neq 1$ ).

\subsection{Harish-Chandra induction and restriction}

Let $L$ be a Levi subgroup of $G$. Let $P$ be a parabolic subgroup and $U$ a Levi complement of $L$ in $P$. We define a functor from the class of $R L$-modules to the class of $R G$-modules by

$$
\mathrm{R}_{L}^{G}: \bmod -R L \rightarrow \bmod -R G, \quad M \mapsto \operatorname{Ind}_{P}^{G} \circ \operatorname{Inf}_{L}^{P} M
$$

where $\operatorname{Ind}_{P}^{G}$ denotes the ordinary induction from $P$ to $G$ and $\operatorname{Inf}_{L}^{P}$ inflation from $L$ to $P$. This functor is called Harish-Chandra induction. The adjoint functor (both left and right adjoint) is defined by

$$
\mathrm{T}_{L}^{G}: \bmod -R G \rightarrow \bmod -R L, \quad N \mapsto \operatorname{Fix}_{U}^{P} \circ \operatorname{Res}_{P}^{G} N
$$

where $\operatorname{Res}_{P}^{G}$ is the ordinary restriction and $\operatorname{Fix}_{U}^{P}$ is the Fix-point functor. The composition is called Harish-Chandra restriction. 
These definitions as well as proofs for adjointness and the following properties can be found in [DiFl].

Quite often it is useful to give the functors in a different form. We write $\hat{U}:=$ $\frac{1}{|U|} \sum_{u \in U} u$. Note that $\hat{U} \in R U$ for all choices of $R$ since $p$ does not divide $\ell$ and $U$ is a $p$-group. Then $\mathrm{R}_{L}^{G} M \cong M \hat{U} \underset{R P}{\otimes} R G$ and $\mathrm{T}_{L}^{G} N \cong N \hat{U}$ as $R G$-respectively $R L$-module.

Proposition 2.1 (Transitivity). Let $L \leq M \leq G$ with $L, M$ Levi subgroups of G. Then

$$
\mathrm{R}_{M}^{G} \circ \mathrm{R}_{L}^{M}=\mathrm{R}_{L}^{G} \text { and } \mathrm{T}_{L}^{M} \circ \mathrm{T}_{M}^{G}=\mathrm{T}_{L}^{G}
$$

Proposition 2.2 (Mackey formula). Let $L, M$ be standard Levi subgroups of $G$ and $N$ an $R L$-module. Then we have an isomorphism of $R$-modules:

$$
\mathrm{T}_{M}^{G} \mathrm{R}_{L}^{G} N \cong \bigoplus_{x \in \mathcal{D}_{L, M}} \mathrm{R}_{L^{x} \cap M}^{M} \mathrm{~T}_{L^{x} \cap M}^{L^{x}} N^{x}
$$

where $\mathcal{D}_{L, M}$ denotes a set of distinguished LxM double coset representatives.

Proposition 2.3. Let $L_{1}, L_{2}$ be conjugate Levi subgroups of $G$. Then the functors $\mathrm{R}_{L_{1}}^{G}$ and $\mathrm{R}_{L_{2}}^{G}$ are naturally equivalent. The same is also true for the functors $\mathrm{T}_{L_{1}}^{G}$ and $\mathrm{T}_{L_{2}}^{G}$.

This was proved independently by Dipper and $\mathrm{Du}([\mathrm{DiDu}])$ and Howlett and Lehrer ([HoLe2]) and shows that we can usually just consider standard Levi subgroups. It also shows, that Harish-Chandra induction and restriction do not depend on the choice of a parabolic subgroup.

Proposition 2.4. $\mathrm{R}_{L}^{G}$ and $\mathrm{T}_{L}^{G}$ are exact functors and map projective modules into projective modules. Furthermore, these functors commute with taking $\ell$-modular reduction.

This is proved in [DiFl], $\S 1$

\subsection{Construction of the irreducible modules}

This subsection gives a brief overview on how to construct the irreducible representations and fixes some necessary notation for later use. In this section, take $R \in\{K, F\}$.

An irreducible $R G$-module $C$ is called cuspidal if $\mathrm{T}_{L}^{G} C=0$ for every Levi subgroup $L \subsetneq G$. The cuspidal modules are the building blocks in the construction of the irreducible modules. We have the following: 
Theorem 2.5 (Cuspidal Modules). Let $s \in G$ be a semisimple element with minimal polynomial of degree $n$. Then there exists an irreducible cuspidal $K G$ module $M_{K}(s,(1))$. The $\ell$-modular reduction $M_{F}(s,(1)):=\overline{M_{K}(s,(1))}$ remains irreducible (and of course cuspidal). The modules $M_{R}\left(s,(1)\right.$ and $M_{R}(t,(1))$ are isomorphic if and only if the $\ell$-regular parts of $s$ and $t$ have the same minimal polynomial.

The construction of $M_{K}(s,(1))$ is due to Gelfand (see [Gel]). It can also be found, together with the rest of the proof, in [Ja3].

Now we take $d, k \in \mathbb{N}$ such that $d k=n$. Let $L \subseteq G$ be the group of block diagonal matrices, with blocks of size $d \times d$ i.e. $L \cong\left(\mathrm{Gl}_{d}(q)\right)^{k} \in \mathcal{L}_{G}$. Let $M:=$ $M_{R}(s,(1))$ be a cuspidal $R \mathrm{Gl}_{d}(q)$-module. Then the tensor product $M^{\otimes k}$ is a cuspidal module for $R L$. We write $M_{R}\left(s,\left(1^{k}\right)\right)$ for the module $\mathrm{R}_{L}^{G} M^{\otimes k}$ and we write $E=\operatorname{End}_{R G}\left(M_{R}\left(s,\left(1^{k}\right)\right)\right)$.

Theorem 2.6. The endomorphism algebra $E$ is the Hecke algebra $\mathcal{H}_{q^{d}, R}\left(S_{k}\right)$ which is an associative algebra with basis $\left\{T_{w} \mid w \in S_{k}\right\}$. The multiplication follows the following rule: if $w \in S_{n}$ and $v=(i, i+1)$ for some $i$ with $1 \leq i \leq n-1$, then

$$
T_{w} T_{v}= \begin{cases}T_{w v} & \text { if } l(w v)=l(w)+1 \\ q^{d} T_{w v}+\left(q^{d}-1\right) T_{w} & \text { otherwise }\end{cases}
$$

The proof can be found in [Di2] or [HoLe1].

Let $\lambda=\left(\lambda_{1}, \lambda_{2}, \ldots, \lambda_{r}\right) \models k$ and write $x_{\lambda}:=\sum_{w \in W_{\lambda}} T_{w}$ where $W_{\lambda}$ denotes the Young subgroup $S_{\lambda_{1}} \times \cdots \times S_{\lambda_{r}}$ of $S_{k}$. We define $M_{R}(s, \lambda):=x_{\lambda} M_{R}\left(s,\left(1^{k}\right)\right)$.

Now we want to construct the so called Specht modules. First we need a nontrivial homomorphism $x_{R}:(\mathrm{GF}(q),+) \rightarrow R^{*}$. For $\mu=n$ let $\theta_{\mu}(u):=x_{R}\left(\sum u_{i, i+1}\right)$ where $u \in U$ and $u_{i, j}$ is the $(i, j)$ entry in $u$ and the sum is taken over those $i$ where $i$ and $i+1$ are in the same row of the standard $\mu$-tableau. We define:

$$
E_{R}^{+}(\mu)=\frac{1}{|U|} \sum_{u \in U} \theta_{\mu}\left(u^{-1}\right) u \quad \text { see }[\mathrm{Ja} 3], 1.3
$$

With this idempotent of $R U$ we define the Specht module $S_{R}(s, \lambda)$ as $M_{R}(s, \lambda) E_{R}^{+}\left(d \lambda^{\prime}\right) R G$.

Theorem 2.7. The module $S_{R}(s, \lambda)$ has a unique maximal $R G$-submodule. In case of $R=K$ this implies that $S_{K}(s, \lambda)$ must be irreducible.

Proposition 2.8. $S_{F}(s, \lambda)$ is an $\ell$-modular reduction of $S_{K}(s, \lambda)$.

Now we find the modular irreducible $F G$-modules as

$$
D_{F}(s, \lambda):=S_{F}(s, \lambda) / S_{F}^{\max }(s, \lambda)
$$


see [Ja3], chapter7 for proofs

A complete classification of the modular irreducibles was first given by Dipper in [Di1],[Di2], later by different methods by James [Ja3]. In [DiJa1] both authors brought their methods together and we use a mixing procedure described there to construct a complete set of inequivalent irreducible $K G$-modules and $F G$ modules:

Choose one root for each monic, irreducible polynomial over $\mathrm{GF}(q)$ except $X$ and call this set of roots $\mathcal{C}$ and well-order it. Let $s_{1}<s_{2}<\cdots<s_{N}$ be elements of $\mathcal{C}$. For $1 \leq i \leq N$ denote by $d_{i}$ the degree of $s_{i}$ over $\mathrm{GF}(q)$ and let $\lambda^{(i)} \vdash k_{i}$. Suppose that $d_{1} k_{1}+\cdots+d_{N} k_{N}=n$. Denote by $L$ the Levi subgroup $\operatorname{Gl}_{d_{1} k_{1}}(q) \times \cdots \times$ $\mathrm{Gl}_{d_{N} k_{N}}(q)$. Then we have a list of all non-isomorphic irreducible $R G$-modules:

(i) $\mathrm{R}_{L}^{G}\left(S_{K}\left(s_{1}, \lambda^{(1)}\right) \otimes \cdots \otimes S_{K}\left(s_{N}, \lambda^{(N)}\right)\right)$ is an irreducible $K G$-module and

(ii) $\mathrm{R}_{L}^{G}\left(D_{F}\left(s_{1}, \lambda^{(1)}\right) \otimes \cdots \otimes D_{F}\left(s_{N}, \lambda^{(N)}\right)\right)$ is an irreducible $F G$-module, provided all the $s_{i}$ are $\ell$-regular.

\subsection{Harish-Chandra series and block structure}

The irreducible and indecomposable modules can be divided into series. On one hand there is the block structure, on the other hand are Harish-Chandra series.

Let $R \in\{K, F\}$. The Harish-Chandra series $S_{R}(L, C)$ is defined to be all the irreducible constituents of hd $\mathrm{R}_{L}^{G}(C)$, where $L$ is a Levi subgroup of $G$ and $C$ is a cuspidal $R L$-module. We have the following theorem due to Hiss [Hi]:

Theorem 2.9. The irreducible $R G$-modules are partitioned into Harish-Chandra series. Two series $S_{R}(L, C)$ and $S_{R}\left(L^{\prime}, C^{\prime}\right)$ are equal, if $L$ is conjugate to $L^{\prime}$ and $C$ and $C^{\prime}$ are conjugate in $N_{G}(L)$.

We do not want to go into the details here, because writing down the series in complete generality requires some additional machinery. This can be found in [Di3], $\S 4$ and $\S 5$. But we do want to give the HC-series of the unipotent block (see below) in the case $e \cdot \ell>n$.

Proposition 2.10. Assume that $e \cdot \ell>n$ and let $s_{1}<s_{2}<\cdots<s_{N}$ with $s_{i} \in \mathcal{C}$ be semisimple $\ell$-elements. Assume $d_{1} k_{1}+\cdots+d_{N} k_{N}=n$ with $d_{i}=\operatorname{deg} s_{i}$. For each $s_{i}$ there is a unique irreducible, cuspidal $\mathrm{Gl}_{d_{i}}(q)$ module $C\left(s_{i}\right)$. Take $L=\mathrm{Gl}_{d_{1}}(q)^{k_{1}} \times \cdots \times \mathrm{Gl}_{d_{N}}(q)^{k_{N}}$ and $C=C\left(s_{1}\right)^{\otimes k_{1}} \otimes \cdots \otimes C\left(s_{N}\right)^{\otimes k_{N}}$. Then the irreducible $K G$-modules in this $H C$-series, also called the ordinary $H C$-series, are given by:

$$
S_{K}(L, C)=\left\{\mathrm{R}_{L}^{G}\left(S_{K}\left(s_{1}, \lambda_{1}\right) \otimes \cdots \otimes S_{K}\left(s_{N}, \lambda_{N}\right)\right) \mid \lambda_{i} \vdash k_{i} \forall i\right\}
$$


For the division of the irreducible FG-modules into series we need $\tilde{L}=\mathrm{Gl}_{e}(q)^{r} \times$ $\mathrm{Gl}_{1}(q)^{s}$ such that $n=e r+s$. The unique cuspidal $F \tilde{L}$-module in this block is $C=D_{F}\left(1,1^{e}\right)^{\otimes r} \otimes D_{F}(1,1)^{\otimes s}$. The $H C$-series, also called the modular $H C$-series, is given by:

$$
S_{F}(\tilde{L}, C)=\left\{\mathrm{R}_{M}^{G}\left(D_{F}\left(1,\left(e \rho^{\prime}\right)^{\prime}\right) \otimes D_{F}(1, \mu)\right) \mid \rho \vdash r, \mu \vdash s \text { e-regular }\right\}
$$

where $M=\mathrm{Gl}_{e r}(q) \times \mathrm{Gl}_{s}(q)$.

We use the connection between the ordinary and the modular Harish-Chandra series to make the following observation.

Proposition 2.11. Let $S_{K}$ be an irreducible $K G$-module and let $S_{F}$ be an $\ell$ modular reduction of $S_{K}$. Furthermore suppose $D_{F}$ is a composition factor of $S_{F}$. Then for any Levi subgroup $L \leq G$ we have that $\mathrm{T}_{L}^{G} S_{K}=0$ implies $\mathrm{T}_{L}^{G} D_{F}=0$. Therefore, if $S_{K}$ lies in the Harish-Chandra series $S_{K}(M, C)$. then $D_{F}$ lies in a Harish-Chandra series $S_{F}\left(M^{\prime}, C^{\prime}\right)$, where $M \leq M^{\prime}$.

Proof. Taking $\ell$-modular reduction is compatible with the HC-restriction functor so $\mathrm{T}_{L}^{G} S_{K}=0$ implies $\mathrm{T}_{L}^{G} S_{F}=0$. Since $\mathrm{T}_{L}^{G}$ is exact we have $\mathrm{T}_{L}^{G} D_{F}=0$ for any composition factor of $S_{F}$.

We know from the construction of the $H C$-series (see [Hi]) that $D_{F} \in S_{F}\left(M^{\prime}, C^{\prime}\right.$ ) implies that $M^{\prime}$ is minimal among the Levi subgroups such that $\mathrm{T}_{M^{\prime}}^{G} D_{F} \neq 0$ and the same holds true for $S_{K} \in S_{K}(M, C)$. So the second claim follows from the first.

The following observation though rather obvious will be quite useful on several occasions for the rest of this paper.

Proposition 2.12. Let $L \leq M$ be standard Levi subgroups of $G$. Suppose that the $D$ is irreducible and belongs to the Harish-Chandra series $S_{F}(L, C)$. Then all composition factors of soc $\mathrm{T}_{M}^{G} D$ and hd $\mathrm{T}_{M}^{G} D$ belong to the Harish-Chandra series $S_{F}(L, C)$ of $M$.

Proof. Let $X$ be any composition factor of soc $\mathrm{T}_{M}^{G} D$ and suppose $X$ belongs to the Harish-Chandra series $S_{F}(\bar{L}, \bar{C})$. Then $\operatorname{Hom}_{F M}\left(\mathrm{R}_{\bar{L}}^{M} \bar{C}, \mathrm{~T}_{M}^{G} D\right) \neq 0$. Therefore by adjointness of functors $\operatorname{Hom}_{F G}\left(\mathrm{R}_{\bar{L}}^{G} \bar{C}, D\right) \neq 0$ and so $D$ belongs to the HarishChandra series $S_{F}(\bar{L}, \bar{C})$. This implies, that $\bar{L}$ is conjugate to $L$ and $\bar{C}$ is conjugate to $C$ and our claim is proved. The claim for hd $\mathrm{T}_{M}^{G} D$ is proved similarly.

A second method to partition the irreducible $R G$-modules (and even the indecomposable ones) is the partition into blocks. The blocks of the general linear groups have been determined by Fong and Srinivasan in [FoSri2]. Here we only consider those irreducibles belonging to the same block. 
Proposition 2.13. Let $\mathrm{R}_{L_{1}}^{G}\left(D_{F}\left(s_{1}, \lambda_{1}\right) \otimes \cdots \otimes D_{F}\left(s_{N}, \lambda_{N}\right)\right)$ and $\mathrm{R}_{L_{2}}^{G}\left(D_{F}\left(t_{1}, \mu_{1}\right) \otimes\right.$ $\left.\cdots \otimes D_{F}\left(t_{M}, \mu_{M}\right)\right)$ be irreducible $F G$-modules as described above. These are in the same $\ell$-block of $G$ if and only if the following conditions hold for all $i$ :

(i) $s_{i}=t_{i}$ with degree $d_{i}$

(ii) $\lambda_{i}$ and $\mu_{i}$ are partitions of the same integer $k_{i}$.

(iii) $\lambda_{i}$ and $\mu_{i}$ have the same $\tilde{e}\left(d_{i}\right)$-core.

This was made explicit in [DiJa2] as was the following proposition.

Proposition 2.14. Let $\mathrm{R}_{L_{1}}^{G}\left(S_{K}\left(s_{1}, \lambda_{1}\right) \otimes \cdots \otimes S_{K}\left(s_{N}, \lambda_{N}\right)\right.$ and $\mathrm{R}_{L_{2}}^{G}\left(S_{K}\left(t_{1}, \mu_{1}\right) \otimes\right.$ $\cdots \otimes S_{K}\left(t_{M}, \mu_{M}\right)$ be irreducible $K G$-modules as defined above. These are in the same $\ell$-block of $G$ if and only if the following conditions hold:

(i) $\sum_{\substack{i=1 \\ s_{i}^{\prime}=u}}^{N}\left(\operatorname{deg} s_{i}\right)\left|\lambda_{i}\right|=\sum_{\substack{j=1 \\ t_{j}^{\prime}=u}}^{M}\left(\operatorname{deg} t_{j}\right)\left|\mu_{j}\right|$ for all -regular $u \in \mathcal{C}$, where $s_{i}^{\prime}$ is the $\ell$-regular part of $s_{i}$.

(ii) For every $s_{i}$ there exists a $t_{j}$ such that the $\tilde{e}\left(\operatorname{deg} s_{i}\right)$-core of $\lambda_{i}$ is the same as the $\tilde{e}\left(\operatorname{deg} t_{j}\right)$-core of $\mu_{j}$ and vice versa.

By a theorem of Broué and Michel ([BrMi]) we know that certain unions of Harish-Chandra series form a union of blocks. We formulate here a version in our special case, with the notation above:

Proposition 2.15. Let $\mathrm{R}_{L_{1}}^{G}\left(D_{F}\left(s_{1}, \lambda_{1}\right) \otimes \cdots \otimes D_{F}\left(s_{N}, \lambda_{N}\right)\right)$ and $\mathrm{R}_{L_{2}}^{G}\left(D_{F}\left(t_{1}, \mu_{1}\right) \otimes\right.$ $\left.\cdots \otimes D_{F}\left(t_{M}, \mu_{M}\right)\right)$ be irreducible $F G$-modules as described above. These are in the same union of $\ell$-blocks if and only if $s_{i}=t_{i}$ for all $i$.

Let $\mathrm{R}_{L_{1}}^{G}\left(S_{K}\left(s_{1}, \lambda_{1}\right) \otimes \cdots \otimes S_{K}\left(s_{N}, \lambda_{N}\right)\right.$ and $\mathrm{R}_{L_{2}}^{G}\left(S_{K}\left(t_{1}, \mu_{1}\right) \otimes \cdots \otimes S_{K}\left(t_{M}, \mu_{M}\right)\right.$ be irreducible $K G$-modules as defined above. These are in the same union of $\ell$-blocks if and only if

$$
\sum_{s_{i}^{\prime}=u}\left(\operatorname{deg} s_{i}\right)\left|\lambda_{i}\right|=\sum_{t_{j}^{\prime}=u}\left(\operatorname{deg} t_{j}\right)\left|\mu_{j}\right| \text { for all } \ell \text {-regular } u \in \mathcal{C} .
$$

So each union of blocks is characterised uniquely by an $\ell$-regular semisimple element.

In an abuse of language we will call the union of blocks characterised by the semisimple element $1 \in G$ the unipotent block.

In this thesis we restrict ourselves to blocks with an Abelian defect group. By this we mean that the union of blocks we are looking at consists of blocks which 
have all Abelian defect groups. Furthermore we restrict ourselves to the unipotent block. But where the first condition is necessary to give us a problem that can be handled, the second is mostly for convenience and to shorten notation. We look again at the case of arbitrary blocks with Abelian defect groups in the last chapter.

From Fong and Srinivasan in [FoSri2] we know that a conjugate of every $\ell$-element of $G$ lies in the defect group of the unipotent block. Therefore the defect group is the $\ell$-Sylow subgroup of $G$. It is Abelian if and only if $e \cdot \ell>n$. (see [Weir]). So we can substitute this condition for the condition of Abelian defect group.

\subsection{Steinberg Module and Gelfand-Graev Module}

In this subsection we consider two important special modules. First we describe the construction of the Gelfand-Graev module for a standard Levi subgroup $L$ of $G$. Let $\chi$ be a linear character of $U \cap L$ such that the restriction to each root subgroup $X_{i} \subseteq L$ is nontrivial. Since $|U \cap L|$ is invertible in $O$, we have the following idempotent in $O L$ :

$$
e_{L}:=\frac{1}{|U \cap L|} \sum_{u \in U \cap L} \chi\left(u^{-1}\right) u
$$

By general theory $\Gamma_{L, O}:=e_{L} O L$ is projective and hence up to isomorphism the only $O L$-lattice in the $K L$-module $e_{L} K L=\Gamma_{L, K}$. We call this module (and its $\ell$-modular reduction $\left.\Gamma_{L, F}\right)$ the Gelfand-Graev module of $L$. It was first introduced by Gelfand and Graev in [GelGr]. From the definition it is easy to calculate that for $L \cong \mathrm{Gl}_{n_{1}}(q) \times \cdots \times \mathrm{Gl}_{n_{r}}(q)$ we have

$$
\Gamma_{L, R} \cong \Gamma_{\mathrm{Gl}_{n_{1}}(q), R} \otimes \cdots \otimes \Gamma_{\mathrm{Gl}_{n_{r}}(q), R}
$$

The Gelfand-Graev module has some interesting properties:

Proposition 2.16. All irreducible components of the Gelfand-Graev module $\Gamma_{G, K}$ occur with multiplicity 1.

Proposition 2.17. Each Harish-Chandra series $S_{K}(L, C)$ has exactly one irreducible $K G$-module with $\Gamma_{G, K}$ in common, which is given by $\mathrm{R}_{L}^{G}\left(S_{K}\left(s_{1},\left(1^{k_{1}}\right)\right) \otimes\right.$ $\left.\cdots \otimes S_{K}\left(s_{r},\left(1^{k_{r}}\right)\right)\right)$.

Proposition 2.18. For any Levi subgroup $L$ of $G$ we have $\mathrm{T}_{L}^{G} \Gamma_{G, R} \cong \Gamma_{L, R}$.

Proofs of these propositions can be found in [Carter] $\S 8$.

Because of Proposition 2.17 we know there is a unique irreducible $K G$ module which the Gelfand-Graev module $\Gamma_{G, K}$ and the Harish-Chandra series $S_{K}\left(T, 1_{T}\right)$ 
have in common. This module is called the Steinberg module and in our previous notation is denoted by $S_{K}\left(1,\left(1^{n}\right)\right)$. The $\ell$-modular reduction $S_{F}\left(1,\left(1^{n}\right)\right)$ is called the modular Steinberg module. Its head is $D_{F}\left(1,\left(1^{n}\right)\right)$. Since $\Gamma_{G, R}$ is projective, the projective indecomposable module with $D_{K}\left(1,\left(1^{n}\right)\right)$ in its head is the unique projective indecomposable module (short PIM) that the unipotent block and the Gelfand-Graev module have in common. We call it the Steinberg-PIM and denote it by $P_{S t}(G)$.

Corollary 2.19. Let $L \cong \mathrm{Gl}_{n_{1}}(q) \times \cdots \times \mathrm{Gl}_{n_{r}}(q)$ be a standard Levi subgroup of G. Then $P_{S t}(L) \cong \mathrm{T}_{L}^{G} P_{S t}(G)$.

Proof. From Propositions 2.17 and 2.18 we know that $P_{S t}(L)$ is a PIM in the Gelfand-Graev module of $L$. On the other hand let $S_{K}(M, C)$ be any HarishChandra series in the unipotent block. Then we use the Mackey formula:

$$
\mathrm{T}_{L}^{G} \mathrm{R}_{M}^{G} C \cong \bigoplus_{x \in \mathcal{D}_{M L}} \mathrm{R}_{M^{x} \cap L}^{L} \mathrm{~T}_{M^{x} \cap L}^{M^{x}} C^{x} \cong \bigoplus_{\substack{x \in \mathcal{D}_{M L} \\ M^{x} \subseteq L}} \mathrm{R}_{M^{x}}^{L} C^{x}
$$

But the $\ell$-regular part of the semisimple element corresponding to $C^{x}$ is the same as the one corresponding to $C$, e.g. 1. This shows that the restriction of any module in the unipotent block of $G$ to a Levi subgroup $L$ lies in the unipotent block of that Levi subgroup.

We use this isomorphism to identify $\mathrm{T}_{L}^{G} P_{S t}(G)$ with $P_{S t}(L)$. This leaves no nontrivial ambiguity since $\operatorname{Aut}_{R L}\left(P_{S t}(L)\right)$ is one-dimensional.

\section{Steinberg-PIM and Harish-Chandra restric- tion}

In this section we want to determine the behaviour of the irreducible constituents of the Steinberg-PIM under Harish-Chandra restriction.

\subsection{The irreducible constituents of the Steinberg-PIM}

In the whole section the relation between $e$, which is the order of $q$ modulo $\ell$ and $e(1)$, which is the smallest integer such that $\ell$ divides $1+q+\cdots+q^{e(1)-1}$ plays an important role. Therefore the case $e=1 \neq e(1)$ is special and will be treated separately.

Proposition 3.1. The $\ell$-modular reduction of the Steinberg module $S_{F}\left(1,\left(1^{n}\right)\right)$ has exactly one composition factor $D_{F}(1, \lambda)$ with $\lambda \vdash n$ an $e(1)$-regular partition. 
All other composition factors are of the form $D_{F}(1, \mu)$ with $\mu \vdash n$ an e(1)-singular partition. The e(1)-regular composition factor $D_{F}(1, \lambda)$ occurs with multiplicity one. Furthermore, $\lambda$ can be calculated using the Mullineux map applied to the partition $(n)$ of $n$.

Proof. We know that the columns of the decomposition matrix of the unipotent block of $G$ indexed by $e(1)$-regular partitions of $n$ are equal to the decomposition matrix of the Hecke algebra $\mathcal{H}_{q, k}\left(S_{n}\right)$ (see [Ja3], Thm 8.1). The Specht module of the Hecke algebra corresponding to $S_{K}\left(1,\left(1^{n}\right)\right)$ is the alternating module of the Hecke algebra $S^{\left(1^{n}\right)}$. But the alternating module is 1-dimensional, i.e. irreducible. Therefore $S^{\left(1^{n}\right)} \cong D^{\lambda}$ for some $\lambda \vdash n e(1)$-regular and so $S_{F}\left(1,1^{n}\right)$ has exactly one $e(1)$-regular composition factor $D_{F}(1, \lambda)$.

To calculate the partition $\lambda$ we have to introduce an outer automorphism of the Hecke algebra:

$$
\sharp: \mathcal{H}_{q, R}\left(S_{n}\right) \rightarrow \mathcal{H}_{q, R}\left(S_{n}\right) \quad T_{v} \mapsto(q-1) T_{1}-T_{v}
$$

where $v$ is a basic transposition in $S_{n}$. This can be extended to products $T_{v_{1}} T_{v_{2}}$ and then linearly to sums. With this we have $\left(S_{K}^{(n)}\right)^{\sharp} \cong S_{K}^{\left(1^{n}\right)}$ (see [DiJa2] Lemma 2.2 ). But this means that the irreducible constituent in the head of $S_{F}^{\left(1^{n}\right)}$ is the image of the trivial module under the Mullineux map as was shown by Brundan $[\mathrm{Bru}]$. For the convenience of the reader we will describe a combinatorial way to calculate the Mullineux map in appendix B.

We want to extend the result above to the Steinberg-PIM. To do this we need to know the ordinary irreducible constituent of said PIM:

Proposition 3.2. Each Harish-Chandra series of ordinary irreducible modules contains exactly one constituent of the Gelfand-Graev module.

Proof. This follows from the fact that in the case of $\mathrm{Gl}_{n}(q)$ Harish-Chandra series and geometric conjugacy classes coincide and that each geometric conjugacy classes contains exactly one constituent of the Gelfand-Graev module (see [Carter],§8).

From now on in this section we assume that $e=e(1)>1$ except when explicitly stated otherwise.

So all ordinary constituents of the Steinberg-PIM except the Steinberg module itself belong to $\mathrm{HC}$-series $S_{K}(L, C)$ with $T \lesseqgtr L$ since $1_{T}$ is the only cuspidal $T$-module in the unipotent block. But by Theorem 2.9 we know that all $D_{F}(1, \lambda)$ with $\lambda \vdash n$ e-regular lie in the Harish-Chandra series $S_{F}\left(T, 1_{T}\right)$. So by Proposition 2.11 the Steinberg module $S_{F}\left(1,\left(1^{n}\right)\right)$ is the only possible source of $e$-regular composition factors and we get the following corollary: 
Corollary 3.3. The Steinberg-PIM $P_{S t}(G)$ has exactly one composition factor $D_{F}(1, \lambda)$ where $\lambda \vdash n$ is e-regular and this composition factor occurs with multiplicity 1. $\lambda$ is the image of $(n)$ under the Mullineux map. We will denote this unique e-regular composition factor of the Steinberg-PIM by $D_{\text {reg }}(n)$.

In case that $n<e$, the Steinberg module remains irreducible under $\ell$-modular reduction. In this case we have $D_{\text {reg }}(n)=D\left(1,1^{n}\right)=S_{F}\left(1,1^{n}\right)$.

Now we can describe the composition factors of $P_{S t}(G)$ in terms of $D_{\text {reg }}(j)$ for $j \leq n$. We use the following Proposition of James ([Ja4], 3.10) which follows from a theorem of Dipper and James ([DiJa1], Thm 5.1):

Proposition 3.4. Let $\mu \vdash n$ and let $\alpha$ and $\rho$ be determined by the conditions, that $\mu^{\prime}=\alpha^{\prime}+e(1) \rho^{\prime}$ and that $\alpha$ is e(1)-regular. Let $s$ be any $\ell$-element of degree $e(1)$ over $\mathrm{GF}(q)$ and let $L \cong \mathrm{Gl}_{|\alpha|}(q) \times \mathrm{Gl}_{e(1)|\rho|}(q)$. Then:

$$
D_{F}(1, \mu) \cong \mathrm{R}_{L}^{G}\left(D_{F}(1, \alpha) \circ D_{F}(s, \rho)\right) \cong \mathrm{R}_{L}^{G}\left(D_{F}(1, \alpha) \circ D_{F}\left(1,\left(e(1) \rho^{\prime}\right)^{\prime}\right)\right)
$$

Theorem 3.5. Let $\mu \vdash n$ and let $\alpha$ and $\rho$ be determined as above with $\alpha \vdash j$ and $\rho \vdash w$. Then $D_{F}(1, \mu)$ is a composition factor of $P_{S t}(G)$ only if $D_{F}(1, \alpha) \cong D_{\text {reg }}(j)$ and $D_{F}\left(1,\left(e \rho^{\prime}\right)^{\prime}\right)$ is a composition factor of $P_{S t}\left(\mathrm{Gl}_{e w}(q)\right)$.

Proof. If $\mu=\alpha$ is $e$-regular, then by Cor. 3.3 there is nothing further to prove. So we can assume $\mu$ to be $e$-singular and therefore $\rho \vdash w \geq 1$.

Suppose $D_{F}(1, \mu)$ is a composition factor of $P_{S t}(G)$. Then by proposition 3.4 $D_{F}(1, \mu) \cong \mathrm{R}_{L}^{G}\left(D_{F}(1, \alpha) \circ D_{F}(s, \rho)\right)$ where $L$ is defined as above. All composition factors of $\mathrm{T}_{L}^{G}\left(D_{F}(1, \mu)\right)$ are composition factors of $\mathrm{T}_{L}^{G} P_{S t}(G) \cong P_{S t}(L)$. By Mackey decomposition we have:

$$
\begin{aligned}
\mathrm{T}_{L}^{G}\left(D_{F}(1, \mu)\right) & \cong \mathrm{T}_{L}^{G} \mathrm{R}_{L}^{G}\left(D_{F}(1, \alpha) \circ D_{F}(s, \rho)\right) \\
& \cong \bigoplus_{x \in \mathcal{D}_{L, L}} \mathrm{R}_{L \cap L^{x}}^{L} \mathrm{~T}_{L \cap L^{x}}^{L^{x}}\left(D_{F}(1, \alpha) \otimes D_{F}(s, \rho)\right)^{x}
\end{aligned}
$$

Since $1 \in \mathcal{D}_{L, L}$ we have that $D_{F}(1, \alpha) \otimes D_{F}(s, \rho)$ is a composition factor of $P_{S t}(L) \cong P_{S t}\left(\mathrm{Gl}_{j}(q)\right) \otimes P_{S t}\left(\mathrm{Gl}_{e w}(q)\right)$. But this implies that $D_{F}(1, \alpha)$ is a composition factor of $P_{S t}\left(\mathrm{Gl}_{j}(q)\right)$ and therefore $D_{F}(1, \alpha) \cong D_{\text {req }}(j)$ by corollary 3.3. And it implies as well that $D_{F}(s, \rho) \cong D_{F}\left(1,\left(e \rho^{\prime}\right)^{\prime}\right)$ is a composition factor of $P_{S t}\left(\mathrm{Gl}_{e w}(q)\right)$.

Naturally we would also like to know which composition factors do occur in the Steinberg-PIM. That question will be answered later. But we can solve one special case:

Corollary 3.6. Let $P=P_{S t}\left(\mathrm{Gl}_{e}(q)\right)$ and $e>1$. The composition factors of $P$ have the form $D_{\text {reg }}(e)$ or $D_{F}\left(1,1^{e}\right)$. Both occur at least once, $D_{\text {reg }}(e)$ exactly once. 
Proof. Since $1^{e}$ is an $e$-singular partition we know that $D_{\text {reg }}(e) \neq D_{F}\left(1,1^{e}\right)$. The composition factor $D_{\text {reg }}(e)$ occurs exactly with multiplicity one by Cor. 3.3. But $D_{F}\left(1,1^{e}\right)$ occurs by the definition since it is the head of the Steinberg-PIM. No other composition factors occur since $D_{F}(1, \mu)$ with $\left(1^{n}\right) \neq \mu \vdash e$ is necessarily $e$-regular and so $D_{F}(1, \mu)$ must be isomorphic to $D_{\text {reg }}(e)$.

\subsection{Harish-Chandra restriction of simple modules in the Steinberg-PIM}

The aim of this subsection is to prove the following first main theorem, which is interesting in its own right, but also plays an important role in determining the structure of the Steinberg-PIM. Throughout this subsection we assume that $e \cdot \ell>n$ and $e=e(1)>1$.

Theorem 3.7. Assume that $e \cdot \ell>n$. Let $D_{F}(1, \mu)$ be an irreducible constituent of the Steinberg-PIM $P_{S t}(G)$. Then for any Levi subgroup $M \in \mathcal{L}_{G}$ we have either $\mathrm{T}_{M}^{G} D_{F}(1, \mu)=0$ or $\mathrm{T}_{M}^{G} D_{F}(1, \mu)$ is a direct sum of simple $F M$-modules.

Before we can prove this, we need to look at some special cases:

Proposition 3.8. Let $L \cong \mathrm{Gl}_{n_{1}}(q) \times \cdots \times \mathrm{Gl}_{n_{r}}(q)$. Then $\mathrm{T}_{L}^{G} D_{\text {reg }}(n) \cong D_{\text {reg }}\left(n_{1}\right) \otimes$ $\cdots \otimes D_{\text {reg }}\left(n_{r}\right)$.

Proof. This is a special case of a theorem of Dipper and Gruber ([DiGr], Theorem 2.25). Here we give a proof which uses the much simpler setting and is therefore more elementary.

We already know that $\mathrm{T}_{L}^{G} P_{S t}(G) \cong P_{S t}(L)$ (Cor. 2.19) and that $P_{S t}(L)$ contains only one $e$-regular composition factor namely $D_{\text {reg }}\left(n_{1}\right) \otimes \cdots \otimes D_{\text {reg }}\left(n_{r}\right)$ (see Cor. 3.3). Since HC-restriction is exact all composition factors of $\mathrm{T}_{L}^{G} D_{\text {reg }}(n)$ are contained in $P_{S t}(L)$. By Prop. 2.12 we know that head and socle of $\mathrm{T}_{L}^{G} D_{\text {reg }}(n)$ consist of composition factors from the series $S_{F}\left(1,1_{T}\right)$. But these are exactly the $e$-regular composition factors, so

$$
\operatorname{hd} \mathrm{T}_{L}^{G} D_{\text {reg }}(n)=\operatorname{soc} \mathrm{T}_{L}^{G} D_{\text {reg }}(n)=\mathrm{T}_{L}^{G} D_{\text {reg }}(n) \cong D_{\text {reg }}\left(n_{1}\right) \otimes \cdots \otimes D_{\text {reg }}\left(n_{r}\right)
$$

Proposition 3.9. Let $\rho \vdash w$ and let $L \cong \mathrm{Gl}_{a}(q) \times \mathrm{Gl}_{b}(q) \in \mathcal{L}_{\mathrm{Gl}_{e w}(q)}$. Then $\mathrm{T}_{L}^{G} D_{F}\left(1,\left(e \rho^{\prime}\right)^{\prime}\right)= \begin{cases}\bigoplus_{\mu \vdash a} \bigoplus_{\nu \vdash b} a_{\mu \nu \rho} D_{F}\left(1,\left(e \mu^{\prime}\right)^{\prime}\right) \otimes D_{F}\left(1,\left(e \nu^{\prime}\right)^{\prime}\right) & \text { if } a / e, b / e \in \mathbb{Z} \\ 0 & \text { otherwise }\end{cases}$ where $a_{\mu \nu \rho}$ are the Littlewood-Richardson coefficients. 
Proof. We have $D_{F}\left(1,\left(e \rho^{\prime}\right)^{\prime}\right) \cong D_{F}(s, \rho)$ (see [DiJa1], Thm. 5.1) where $s$ is a semisimple $\ell$-element of degree $e$ as above. But since $e \ell>n$ the decomposition matrix is the identity matrix and so we also have that $D_{F}(s, \rho) \cong S_{F}(s, \rho)$. Since $S_{F}(s, \rho)$ lies in the a Harish-Chandra series coming from $\mathrm{Gl}_{e}(q)^{w}$ the second case is clear with Mackey decomposition. For the first case, we have

$$
\mathrm{T}_{L}^{G} S_{F}(s, \rho) \cong \overline{\mathrm{T}_{L}^{G} S_{K}(s, \rho)} \cong \bigoplus_{\mu \vdash(a / e)} \bigoplus_{\nu \vdash(b / e)} a_{\mu \nu \rho} S_{F}(s, \mu) \otimes S_{F}(s, \nu)
$$

This comes from the fact that HC-restriction of Specht modules is governed by the Littlewood-Richardson rule (see [DiJa2] $\S 7$ ). The rest follows, since $S_{F}(s, \mu) \cong$ $D_{F}\left(1,\left(e \mu^{\prime}\right)^{\prime}\right)$ and $S_{F}\left(s_{\nu}\right) \cong D_{F}\left(1,\left(e \nu^{\prime}\right)^{\prime}\right)$ as above.

By induction on the number of factors of the Levi subgroup we get the following, more general corollary:

Corollary 3.10. Let $\rho \vdash w$ and let $L \in \mathcal{L}_{G}$. Then $\mathrm{T}_{L}^{G} D_{F}\left(1,\left(e \rho^{\prime}\right)^{\prime}\right)$ is either 0 or a direct sum of simple modules.

The following technical Lemmas are needed for the proof of the main theorem:

Lemma 3.11. Let $I, J \subseteq \Delta$ and let $x \in \mathcal{D}_{I, J}$ be a distinguished double coset representative. Suppose for $\alpha_{i} \in I$ we have $w \in W_{J}$ such that $\alpha_{i} x w \in \Phi^{-}$. Then $\alpha_{i} x=\alpha_{j} \in J$.

Proof. this is Lemma 2.71 in [Carter]

Lemma 3.12. Let $x \in S_{n}$ be arbitrary. Suppose that $\{1, \ldots, a\}$ are fixed points under the action of $x$ on the set $\{1, \ldots, n\}$ and suppose that $(a+1) x=y \neq(a+1)$. Then the number of displacements of $x(a+1, a+2, \ldots, y)$ is smaller than the number of displacements of $x$.

Proof. Without loss of generality we can assume that $a+1=1$. Let $1 \leq z_{1}<$ $z_{2} \leq n$. Now suppose $z_{1} x<z_{2} x$. If $z_{2} x>y$, then $z_{2} x$ is fixed under the action of the cycle $\pi=(1,2, \ldots, y)$ and therefore $z_{1} x \pi<z_{2} x \pi$. If $z_{2} x \leq y$ then $z_{2} x \neq y$ since $y=1 x$ and $z_{1}<z_{2}$. But this implies $z_{1} x \pi=z_{1} x+1<z_{2} x+1=z_{2} x \pi$. This shows that $(1,2, \ldots, y)$ creates no additional displacements. But clearly for any $1<z_{2}$ with $z_{2} x<y$ we have that $1 x>z_{2} x$ but $1 x \pi=1<z_{2} x \pi$ so $x \pi$ has less displacements than $x$.

Lemma 3.13. Let $L=\mathrm{Gl}_{r}(q) \times \mathrm{Gl}_{n-r}(q)$ and $M=\mathrm{Gl}_{s}(q) \times \mathrm{Gl}_{n-s}(q)$ and let $x \in \mathcal{D}_{L, M}$ be a distinguished double coset representative. Then

$$
\begin{gathered}
L^{x} \cap M=\mathrm{Gl}_{a}(q) \times \mathrm{Gl}_{s-a}(q) \times \mathrm{Gl}_{c}(q) \times \mathrm{Gl}_{n-s-c}(q) \\
L \cap M^{x^{-1}}=\mathrm{Gl}_{a}(q) \times \mathrm{Gl}_{c}(q) \times \mathrm{Gl}_{s-a}(q) \times \mathrm{Gl}_{n-s-c}(q)
\end{gathered}
$$

where $0 \leq a \leq s, 0 \leq c \leq n-s$ and $a+c=r$. Moreover, the action of $x^{-1}$ on $L^{x} \cap M$ exchanges $\mathrm{Gl}_{s-a}(q)$ and $\mathrm{Gl}_{c}(q)$. 
Proof. There is a 1-1 correspondence between standard Levi subgroups and the simple roots contained in them which is compatible with the action of the Weyl group on both. So we will prove this Lemma in the language of simple roots and the action of the Weyl group:

$$
\begin{gathered}
L \leftrightarrow I:=\left\{\alpha_{1}, \ldots, \alpha_{r-1}, \alpha_{r+1}, \ldots, \alpha_{n-1}\right\} \\
M \leftrightarrow J:=\left\{\alpha_{1}, \ldots, \alpha_{s-1}, \alpha_{s+1}, \ldots, \alpha_{n-1}\right\}
\end{gathered}
$$

We have to consider two cases:

First suppose $r \leq s$. Let $0 \leq a \leq r-1$ be minimal with $(a+1) x \neq a+1$. If no such $a$ exists, then $\{1, \ldots, r\}$ are fixed points of $x$. Therefore $x \in W_{I} \cap \mathcal{D}_{I, J}=\{1\}$. In this sub-case $I x=I$ and $L \cap M=\mathrm{Gl}_{r}(q) \times \mathrm{Gl}_{s-r}(q) \times \mathrm{Gl}_{n-s}(q)$. But if such $a$ exists, then $(a+1) x=s+1$. This is shown as follows: assume $(a+1) x=y \leq s$. Then $x(y, a+1, a+2, \ldots, y-1)$ is an element of the same double coset as $x$ with less displacements then $x$ as seen in Lemma 3.12, which is a contradiction to $x$ being a distinguished double coset representative. Assume $(a+1) x=y>s+1$. Then $x(y, s+1, s+2, \ldots, y-1)$ is in the same double coset with less displacements, which is the same contradiction as above. Since $x$ is a distinguished double coset representative, we see that $\alpha_{a+1} x, \ldots, \alpha_{r-1} x$ lie in $\Phi_{J}$ and therefore by Lemma 3.11 in $J$. But this implies $\alpha_{a+1} x=\alpha_{s+1}, \ldots, \alpha_{r-1} x=\alpha_{s+c-1}$. Let $b>r$ be maximal with $b x \neq x$. Because $(a+1) x=s+1$ we have $b>s$. With the same arguments as above we get $b x=s$ and $b x=s, \ldots,(r+1) x=s+(r+1)-b$. But since $x$ fixes everything larger than $b$ and everything smaller than $a$ we get $b=s+c$ and $x$ is given by:

$$
x=\left(\begin{array}{cccccc}
a+1 & \ldots & r & r+1 & \ldots & s+c \\
s+1 & \ldots & s+c & a+1 & \ldots & s
\end{array}\right)
$$

Therefore

$$
I x \cap J=\left\{\alpha_{1}, \ldots, \alpha_{a-1}, \alpha_{a+1}, \ldots, \alpha_{s-1}, \alpha_{s+1}, \ldots, \alpha_{s+c-1}, \alpha_{s+c+1}, \ldots, \alpha_{n-1}\right\}
$$

as claimed. With the explicit description of $x$ we see that

$$
I \cap J x^{-1}=\left\{\alpha_{1}, \ldots, \alpha_{a-1}, \alpha_{a+1}, \ldots, \alpha_{r-1}, \alpha_{r+1}, \ldots, \alpha_{s+c-1}, \alpha_{s+c+1}, \ldots, \alpha_{n-1}\right\}
$$

and that we got from $I x \cap J$ to $I \cap J x^{-1}$ by exchanging the to middle blocks. The case $r>s$ can be shown with a similar argument.

At last we have gathered everything we need to proof Theorem 3.7:

Theorem 3.7. Without loss of generality we can assume $M=\mathrm{Gl}_{s}(q) \times \mathrm{Gl}_{n-s}(q)$. The case that $\mu$ is $e$-regular is covered by Prop. 3.8. So we may write $\mu^{\prime}=\alpha^{\prime}+e \rho^{\prime}$ 
with $\alpha \vdash r$-regular and $\rho \vdash w \geq 1$. We have $D_{F}(1, \mu) \cong \mathrm{R}_{L}^{G}\left(D_{F}(1, \alpha) \otimes\right.$ $\left.D_{F}\left(1,\left(e \rho^{\prime}\right)^{\prime}\right)\right)$ with $L=\mathrm{Gl}_{r}(q) \times \mathrm{Gl}_{n-r}(q)$. Now we use Mackey decomposition:

$$
\begin{aligned}
\mathrm{T}_{M}^{G} D_{F}(1, \mu) & \cong \mathrm{T}_{M}^{G} \mathrm{R}_{L}^{G}\left(D_{F}(1, \alpha) \otimes D_{F}\left(1,\left(e \rho^{\prime}\right)^{\prime}\right)\right) \\
& \cong \bigoplus_{x \in \mathcal{D}_{L, M}} \mathrm{R}_{L^{x} \cap M}^{M} \mathrm{~T}_{L^{x} \cap M}^{L^{x}}\left(D_{F}(1, \alpha) \otimes D_{F}\left(1,\left(e \rho^{\prime}\right)^{\prime}\right)\right)^{x}
\end{aligned}
$$

Since $x \in \mathcal{D}_{L, M}$ we know by Lemma 3.13 that

$$
L \cap M^{x^{-1}}=\underbrace{\mathrm{Gl}_{a}(q) \times \mathrm{Gl}_{c}(q)}_{:=X} \times \underbrace{\mathrm{Gl}_{s-a}(q) \times \mathrm{Gl}_{n-s-c}(q)}_{:=Y}
$$

This gives

$$
\mathrm{T}_{L \cap M^{x^{-1}}}^{L}\left(D_{F}(1, \alpha) \otimes D_{F}\left(1,\left(e \rho^{\prime}\right)^{\prime}\right)\right)=\left(\mathrm{T}_{X}^{\mathrm{Gl}}(q) D_{F}(1, \alpha)\right) \otimes\left(\mathrm{T}_{Y}^{\mathrm{Gl}_{n-r}(q)} D_{F}\left(1,\left(e \rho^{\prime}\right)^{\prime}\right)\right)
$$

But the first factor is $D_{\text {reg }}(a) \otimes D_{\text {reg }}(c)$ by prop. 3.8 and the second factor is zero or semisimple by prop. 3.9. $x$ acts on the module the same way as on the groups, permuting the blocks and we get that each factor has the following form for some $\beta \vdash \frac{s-a}{e}, \gamma \vdash \frac{n-s-c}{e}$ :

$$
\begin{aligned}
& \mathrm{R}_{L^{x} \cap M}^{M}\left(D_{r e g}(a) \otimes D_{F}\left(1,\left(e \beta^{\prime}\right)^{\prime}\right) \otimes\right.\left.D_{r e g}(c) \otimes D_{F}\left(1,\left(e \gamma^{\prime}\right)^{\prime}\right)\right) \\
&=\left(\mathrm{R}_{\mathrm{Gl}_{a}(q) \times \mathrm{Gl}_{s-a}(q)}^{\mathrm{Gl}_{s}(q)} D_{r e g}(a) \otimes D_{F}\left(1,\left(e \beta^{\prime}\right)^{\prime}\right)\right) \\
& \qquad\left(\mathrm{R}_{\mathrm{Gl}_{c}(q) \times \mathrm{Gl}_{n-s-c}(q)}^{\mathrm{Gl}_{n-s}(q)} D_{r e g}(c) \otimes D_{F}\left(1,\left(e \gamma^{\prime}\right)^{\prime}\right)\right)
\end{aligned}
$$

But the two induced modules are simple by Prop. 3.4 and this completes the proof.

The proof of the theorem is constructive, so in principal it can be used to calculate $\mathrm{T}_{L}^{G} D_{F}(1, \mu)$ for any composition factor $D_{F}(1, \mu)$ of $P_{S t}(G)$. But we can also use the proof to get the converse of Theorem 3.5:

Proposition 3.14. Let $\mu \vdash n$ such that $\mu^{\prime}=\alpha^{\prime}+e \rho^{\prime}$ with $\alpha$ e-regular. Suppose $D_{F}(1, \alpha)=D_{\text {reg }}(|\alpha|)$ and $D_{F}\left(1,\left(e \rho^{\prime}\right)^{\prime}\right)$ is a composition factor of $P_{S t}\left(\mathrm{Gl}_{e|\rho|}(q)\right)$. Then $D_{F}(1, \mu)$ is a composition factor of $P_{S t}(G)$.

Proof. Let $L=\mathrm{Gl}_{|\alpha|}(q) \times \mathrm{Gl}_{e|\rho|}(q)$. Since $P_{S t}(L)=\mathrm{T}_{L}^{G} P_{S t}(G)$, every composition factor of $P_{S t}(L)$ is a composition factor of $\mathrm{T}_{L}^{G} D_{F}(1, \lambda)$ for some $\lambda \vdash n$, where $D_{F}(1, \lambda)$ is a composition factor of $P_{S t}(G)$. Since $D:=D_{\text {reg }}(|\alpha|) \otimes D_{F}\left(1,\left(e \rho^{\prime}\right)^{\prime}\right)$ is a composition factor of $P_{S t}(L)$ we conclude that $D$ is a composition factor of $\mathrm{T}_{L}^{G} D_{F}(1, \lambda)$ for some composition factor $D_{F}(1, \lambda)$. But the proof of Theorem 3.7 shows that $\lambda=\mu$. 
We get one more corollary from the proof of the theorem which will be useful later on. For this we need the following notation. Let $L=\mathrm{Gl}_{m}(q)^{r}$ and let $X, Y$ be two different $F \mathrm{Gl}_{m}(q)$-modules. Let $M=X^{\otimes \bar{r}} \otimes Y^{\otimes t}$ with $\bar{r}+t=r$ be an $F L$-module. We denote by $\bar{M}$ the direct sum over all different permutations of the factors $X$ and $Y$. For example, if $M=X \otimes X \otimes Y$, then we have

$$
\bar{M}=(X \otimes X \otimes Y) \oplus(X \otimes Y \otimes X) \oplus(Y \otimes X \otimes X) .
$$

We also expand the notation to the situation, where a fixed factor $Z$ is tensored to the end of each summand. So

$$
\overline{X \otimes Y} \otimes Z:=(X \otimes Y \otimes Z) \oplus(Y \otimes X \otimes Z) .
$$

Corollary 3.15. Let $L=\mathrm{Gl}_{e}(q)^{r} \times \mathrm{Gl}_{1}(q)^{s}$ such that $e>s$ and let $D_{F}(1, \mu)$ be a composition factor of $P_{S t}(G)$. Suppose that $\mu \vdash n$ is a partition of $n$ with $\mu^{\prime}=\alpha^{\prime}+e \rho^{\prime}$ such that $\alpha \vdash(e t+s)$ is e-regular and $\rho \vdash \bar{r}$. Then $\mathrm{T}_{L}^{G} D_{F}(1, \mu)$ consists of $m(\rho)$ copies of the following direct summand

$$
\overline{D_{F}\left(1,1^{e}\right)^{\otimes \bar{r}} \otimes D_{\text {reg }}(e)^{\otimes t}} \otimes D_{\text {reg }}(1)^{\otimes s}
$$

where $m(\rho)$ denotes the multiplicity of $D_{F}\left(1,1^{e}\right)^{\otimes \bar{r}}$ in $\mathrm{T}_{\mathrm{Gl}_{e}(q)^{\bar{r}}}^{\mathrm{Gl}_{\bar{r}}(q)} D_{F}\left(1,\left(e \rho^{\prime}\right)^{\prime}\right)$.

Proof. Since $\mathrm{T}_{L}^{G} D_{F}(1, \mu)$ is semisimple and $D_{F}(1, \mu)$ belongs to the HarishChandra series $S_{F}\left(\mathrm{Gl}_{e}(q)^{\bar{r}} \times \mathrm{Gl}_{1}(q)^{e t+s}, D_{F}\left(1,1^{e}\right)^{\otimes \bar{r}} \otimes D_{\text {reg }}(e)^{\otimes t} \otimes D_{\text {reg }}(1)^{\otimes s}\right)$ all composition factors of $\mathrm{T}_{L}^{G} D_{F}(1, \mu)$ belong to this Harish-Chandra series viewed as series of $F L$ (see Prop. 2.12). Since the only possible composition factors of $P_{S t}\left(\mathrm{Gl}_{e}(q)\right)$ are $D_{F}\left(1,1^{e}\right)$ and $D_{\text {reg }}(e)$ (by Cor. 3.6) we know that any composition factor has to be some permutation of

$$
D_{F}\left(1,1^{e}\right)^{\otimes a} \otimes D_{r e g}(e)^{\otimes b} \otimes D_{r e g}(1)^{\otimes s}
$$

with $a+b=r$. But $D_{F}\left(1,1^{e}\right)$ is a cuspidal $F \mathrm{Gl}_{e}(q)$-module and so the number $a$ determines the Harish-Chandra series. Because all composition factors are in the Harish-Chandra series above we know that $a=\bar{r}$ and therefore $b=t$.

We denote by $X$ the module $D_{F}\left(1,1^{e}\right)^{\otimes \bar{r}} \otimes D_{\text {reg }}(e)^{\otimes t} \otimes D_{\text {reg }}(1)^{\otimes s}$. Let $Y$ be any permutation of the $\mathrm{Gl}_{e}(q)$ factors of $X$. Since $\mathrm{T}_{L}^{G} D_{F}(1, \mu)$ is semisimple we can count the multiplicity of the composition factors by calculating the dimension of Hom spaces. We get:

$$
\begin{aligned}
\operatorname{Hom}_{F L}\left(Y, \mathrm{~T}_{L}^{G} D_{F}(1, \mu)\right) \cong \operatorname{Hom}_{F G}\left(\mathrm{R}_{L}^{G} Y, D_{F}(1, \mu)\right) \\
\cong \operatorname{Hom}_{F G}\left(\mathrm{R}_{L}^{G} X, D_{F}(1, \mu)\right) \cong \operatorname{Hom}_{F L}\left(X, \mathrm{~T}_{L}^{G} D_{F}(1, \mu)\right)
\end{aligned}
$$

The first and third isomorphism is true by adjointness of functors and the second by the fact that $\mathrm{R}_{L}^{G} X \cong \mathrm{R}_{L}^{G} Y$ (see Prop. 2.3). So $X$ and $Y$ have the same multiplicity in $\mathrm{T}_{L}^{G} D_{F}(1, \mu)$. 
To calculate the multiplicity of $X$ in $\mathrm{T}_{L}^{G} D_{F}(1, \mu)$ we need to look more closely at the proof of Theorem 3.7. We take $M:=\mathrm{Gl}_{e \bar{r}}(q) \times \mathrm{Gl}_{e t+s}(q)$. Then $D_{F}(1, \mu) \cong$ $\mathrm{R}_{M}^{G} D_{F}\left(1,\left(e \rho^{\prime}\right)^{\prime}\right) \otimes D_{\text {reg }}(e t+s)$. We have by Mackey decomposition

$$
\begin{aligned}
Z:=\mathrm{T}_{M}^{G} \mathrm{R}_{M}^{G} D_{F}\left(1,\left(e \rho^{\prime}\right)^{\prime}\right) \otimes D_{\text {reg }}(e t+s) & \\
& \cong \bigoplus_{x \in \mathcal{D}_{M, M}} \mathrm{R}_{M^{x} \cap M}^{M} \mathrm{~T}_{M^{x} \cap M}^{M^{x}}\left(D_{F}\left(1,\left(e \rho^{\prime}\right)^{\prime}\right) \otimes D_{r e g}(e t+s)\right)^{x}
\end{aligned}
$$

Now the proof of Theorem 3.7 shows that $X$ (with $D_{\text {reg }}(e t+s)$ at the end) can only occur as composition factor in those summands of $\mathrm{T}_{L}^{M} Z$ where $x=1$. And since $\mathrm{T}_{\mathrm{Gl}_{e}(q)^{t} \times \mathrm{Gl}_{1}(q)^{s}}^{\mathrm{Gl}_{e+s}(q)} D_{\text {reg }}(e t+s)=D_{\text {reg }}(e)^{\otimes t} \otimes D_{\text {reg }}(1)^{\otimes s}$ the multiplicity of $X$ in $\mathrm{T}_{L}^{G} D_{F}(1, \mu)$ is equal to the multiplicity of $D_{F}\left(1,1^{e}\right)^{\otimes \bar{r}}$ in $\mathrm{T}_{\mathrm{Gl}_{e}(q)^{\bar{r}}}^{\mathrm{Gl}_{\bar{r}}(q)} D_{F}\left(1,\left(e \rho^{\prime}\right)^{\prime}\right)$.

\subsection{The special Case $e=1$}

With the additional assumption that $e \cdot \ell>n$ this special case is a corollary of the facts that the upper part of the decomposition matrix in this case is just the identity matrix.

Proposition 3.16. Let $D(1, \mu)$ be any irreducible $F G$-module and let $L$ be a standard Levi subgroup of $G$. Suppose that $e=1$ and $\ell>n$. Then $\mathrm{T}_{L}^{G} D(1, \mu)$ is semisimple or zero.

Proof. Under the special assumptions of the proposition we know that the decomposition matrix of the unipotent block in the upper part is just the identity matrix, i.e. $S_{F}(1, \mu) \cong D_{F}(1, \mu)$ for any partition $\mu \vdash n$ (see [Ja4], Thm 6.4). But HC-restriction of a Specht module gives a direct sum of Specht modules for the Levi subgroup. The exact factors can be calculated using the LittlewoodRichardson rule (see [DiJa2], §7). And these Specht modules in turn are irreducible since the upper part of the decomposition matrix of $\mathrm{Gl}_{n_{i}}(q)$ is still the identity matrix for any $n_{i}<n$.

So we see that our main theorem of this section (Thm. 3.7) is true without the assumption that $e>1$.

\section{The Loewy series of a PIM}

\subsection{General Remarks}

There are two Loewy series, called upper and lower series or radical series and socle series. The knowledge of one often gives the knowledge of the other, provided 
the module we are looking at is self-dual. We will show this well known facts for the convenience of the reader.

Let $R \in\{K, F\}$. For any $R G$-module $M$ we define the dual module $M^{*}:=$ $\operatorname{Hom}_{R}(M, R)$ as $R$-vector space. Now we let $G$ act by $(\alpha g)(m):=\alpha\left(m g^{-1}\right)$ and thus turn $M^{*}$ into a right $R G$-module.

Proposition 4.1. Taking the dual is an exact, contravariant functor from the category of finite-dimensional $R G$-modules onto itself. If $P$ is a projective $R G$ module, so is $P^{*}$.

Proof. It is clear that $\operatorname{Hom}_{R}(, R)$ is contravariant. Let $f: M \rightarrow N$ be an $R G$ homomorphism. Then $f^{*}: N^{*} \rightarrow M^{*}, \alpha \mapsto \alpha \circ f$ is also an $R G$-homomorphism:

$$
\begin{aligned}
\left(f^{*}(\alpha \cdot g)(m)\right. & =((\alpha \cdot g) \circ f)(m)=(\alpha \cdot g)(f(m))=\alpha\left(f(m) g^{-1}\right)=\alpha\left(f\left(m g^{-1}\right)\right) \\
& =((\alpha \circ f) \cdot g)(m)=\left(f^{*}(\alpha) \cdot g\right)(m)
\end{aligned}
$$

The functor is also exact, since the underlying functor of vector spaces is exact. Furthermore $R G \cong R G^{*}$ by the anti-automorphism of $R G$, which maps $g \mapsto g^{-1}$. This also shows, that $P$, which is a direct summand of $R G^{n}$ is mapped to a direct summand $P^{*}$ of $\left(R G^{*}\right)^{n} \cong R G^{n}$.

Proposition 4.2. Let $M$ be a finite-dimensional $R G$-module. Then we have the following isomorphisms:

$$
\begin{gathered}
\operatorname{hd}\left(M^{*}\right) \cong(\operatorname{soc} M)^{*} \quad \operatorname{soc}\left(M^{*}\right) \cong(\operatorname{hd} M)^{*} \\
J\left(M^{*}\right) \cong(M / \operatorname{soc} M)^{*} \quad(J(M))^{*} \cong M^{*} / \operatorname{soc}\left(M^{*}\right)
\end{gathered}
$$

This shows, that factors of the socle series of $M$ are the same as the factors of the radical series of $M^{*}$ with the order reversed.

Proof. We show the first and the last isomorphism. The other two are proved similarly. We have

$$
\begin{gathered}
0 \rightarrow \operatorname{soc} M \rightarrow M \rightarrow M / \operatorname{soc} M \rightarrow 0 \\
0 \leftarrow(\operatorname{soc} M)^{*} \leftarrow M^{*} \leftarrow(M / \operatorname{soc} M)^{*} \leftarrow 0
\end{gathered}
$$

But $(\operatorname{soc} M)^{*}$ is semisimple, therefore contained in the head of $M^{*}$. And $(\operatorname{soc} M)^{*} \subsetneq$ hd $M^{*}$ gives $\left(\text { hd } M^{*}\right)^{*} \subsetneq \operatorname{soc} M$. This is a contradiction to the fact, that taking the dual maps irreducibles into irreducibles and so does not change the number of composition factors. So $(\operatorname{soc} M)^{*} \cong$ hd $M^{*}$ and from the above equation follows also $(M / \operatorname{soc} M)^{*} \cong J\left(M^{*}\right)$.

The last conclusion follows from these isomorphisms. 
The irreducible $F G$-modules in the unipotent block have the nice property of being self-dual. This in turn implies that the dual of a PIM $P$ in this block is again the same PIM. This is shown by the work of James in [Ja2], chapter 11. With these propositions in mind we see that calculating the radical series of a PIM or calculating the socle series gives the same information. So we will work in whatever setting is more convenient.

The general idea for the calculation of the Loewy series is the following. First we search for a projective generator $P$ of the block. Then by the Morita theorems (see e.g. [CuRe1]) we have an isomorphism of lattices between the submodule lattice of $P$ and the lattice of right ideals in $E=\operatorname{End}_{R G}(P)$. Then we decompose $E$ and map everything back. Unfortunately, in general we do not have an accessible projective generator and the decomposition of $E$ is also often very hard.

\subsection{Cyclic Defect Groups}

In this subsection we consider the case where all defect groups are cyclic. This is equivalent to the case that $n<2 e$. From now on we restrict ourselves to an actual block with a cyclic defect group. There can be more than one (and blocks with defect 0) in our unipotent block.

From a result of Fong and Srinivasan in [FoSri1] we get:

Proposition 4.3. The Brauer tree of a block with cyclic defect group in $\mathrm{Gl}_{n}(q)$ is an open polygon of length $(e+1)$ with the exceptional characters at one end. Furthermore all PIMs in one actual block are labelled by partitions of $n$ with the same e-core.

This information can be plugged into the main theorem of Peacock ([Pea]) about the submodule lattice of a PIM in a block with cyclic defect group:

Theorem 4.4. Let $P_{1}, \ldots P_{e}$ be the PIMs in a block with cyclic defect group, the order given by the lexicographic order of the partitions, which characterise the PIMs. Let $V_{1}, \ldots, V_{e}$ be their respective heads. Then the submodule lattices take the following form (with $i=2, \ldots, e-1$ ): 
1. In case $e=1$ we have $P_{1}$
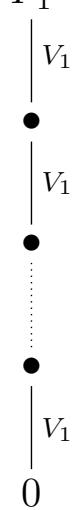

2. In case $e>1$ we have the following:

(i)



(ii)

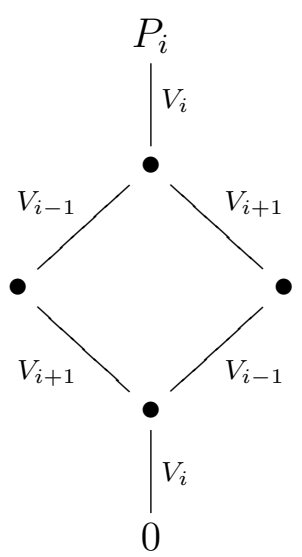


(iii)

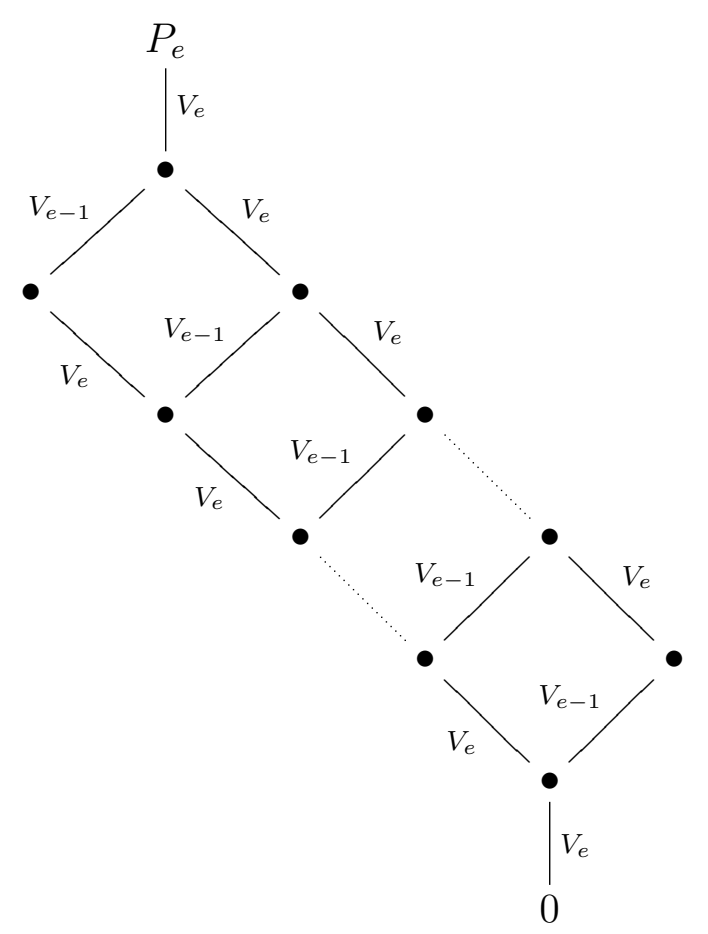

From this information we can write down the various Loewy series and also the structure of the endomorphism ring of any PIM. We do this explicitly for the Steinberg-PIM in the case where $G=\mathrm{Gl}_{e}(q)$ and $e>1$.

Corollary 4.5. Let $G=\mathrm{Gl}_{e}(q)$ and assume $e>1$. Then the radical series and the socle series of the Steinberg-PIM $P_{S t}(G)$ take the following form:

$$
\begin{array}{cc}
D_{F}\left(1,1^{e}\right) & D_{F}\left(1,1^{e}\right) \\
D_{F}\left(1,1^{e}\right) \oplus D_{\text {reg }}(e) & D_{F}\left(1,1^{e}\right) \\
D_{F}\left(1,1^{e}\right) & \vdots \\
\vdots & D_{F}\left(1,1^{e}\right) \\
D_{F}\left(1,1^{e}\right) & D_{F}\left(1,1^{e}\right) \oplus D_{\text {reg }}(e) \\
D_{F}\left(1,1^{e}\right) & D_{F}\left(1,1^{e}\right) \\
\text { radical series } & \text { socle series }
\end{array}
$$

Proof. The Steinberg-PIM corresponds to the exceptional characters since it is the only PIM whose Loewy length is not bounded by 3 . The identification of the factor $V_{e}$ and $V_{e-1}$ comes from the fact that $P_{S t}(G)$ has only the composition factors $D_{\text {reg }}(e)$ and $D_{F}\left(1,1^{e}\right)$ (see Cor. 3.6) and that $V_{e-1}$ occurs in $P_{e}$ with multiplicity 1 as does $D_{\text {reg }}(e)$ in $P_{S t}(G)$.

Note that in the extreme case where the number of composition factors 
of type $D_{F}\left(1,1^{e}\right)$ in $P_{S t}\left(\mathrm{Gl}_{e}(q)\right)$ is exactly 2 , both series take on the form

$$
\begin{gathered}
D_{F}\left(1,1^{e}\right) \\
D_{\text {reg }}(e) \\
D_{F}\left(1,1^{e}\right)
\end{gathered}
$$

Corollary 4.6. Let $P$ be any PIM in a block with cyclic defect group of $G$. Then $\operatorname{End}_{F G}(P) \cong F[X] / X^{k}$, where $k$ is the number of composition factors of $P$ equal to hd $P$.

\subsection{The structure of $P_{S t}(L)$ and $\operatorname{End}_{F L}\left(P_{S t}(L)\right)$}

Let $L$ be the standard Levi subgroup $\mathrm{Gl}_{e}(q)^{r} \times \mathrm{Gl}_{1}(q)^{s}$ of $G$ with $s<e$. From the previous subsection we know the structure of $P_{S t}\left(\mathrm{Gl}_{e}(q)\right)$. We also know if $e>1$, then $P_{S t}\left(\mathrm{Gl}_{1}(q)\right)=D_{F}(1,(1))$. But $P_{S t}(L) \cong P_{S t}\left(\mathrm{Gl}_{e}(q)\right)^{\otimes r} \otimes P_{S t}\left(\mathrm{Gl}_{1}(q)\right)^{\otimes s}$, so all we need is an algorithm to calculate the structure of $P$ and the endomorphism ring of $P$ from the structure of its factors.

From general theory (see [CuRe1], Lemma 10.37) we get:

Proposition 4.7. We have

$$
\begin{aligned}
\operatorname{End}_{F L}\left(P_{S t}(L)\right) & \cong \operatorname{End}_{F \mathrm{Gl}_{e}(q)}\left(P_{S t}\left(\mathrm{Gl}_{e}(q)\right)\right)^{\otimes r} \otimes \operatorname{End}_{F \mathrm{Gl}_{1}(q)}\left(P_{S t}\left(\mathrm{Gl}_{1}(q)\right)\right)^{\otimes s} \\
& \cong \operatorname{End}_{F \mathrm{Gl}_{e}(q)}\left(P_{S t}\left(\mathrm{Gl}_{e}(q)\right)\right)^{\otimes r} .
\end{aligned}
$$

We also want to calculate the radical series of $P_{S t}(L)$ and the radical series of $\operatorname{End}_{F L}\left(P_{S t}(L)\right)$. The procedure is similar, since the structure is similar. To simplify the notation, we just write down the case of two factors. This is folklore but for the convenience of the reader we supply a proof.

Proposition 4.8. Let $A, B$ be finite dimensional F-algebras such that $A / J(A)$ and $B / J(B)$ are split semisimple. Furthermore let $M \otimes N$ be an outer $A \otimes B$ module. Then the $k^{\text {th }}$ radical is given by

$$
J^{k}(M \otimes N)=\sum_{i=0}^{k} J^{i}(M) \otimes J^{k-i}(N)
$$

and the $k^{\text {th }}$ layer in the radical series is given by

$$
J^{k-1}(M \otimes N) / J^{k}(M \otimes N) \cong \bigoplus_{i=0}^{k-1} J^{i}(M) / J^{i+1}(M) \otimes J^{k-1-i}(N) / J^{k-i}(N)
$$

Proof. We want to show

$$
J(M \otimes N)=J(M) \otimes N+M \otimes J(N)
$$


This is a consequence of the fact that with our assumptions on $A$ and $B$ we have $J(A \otimes B)=J(A) \otimes B+A \otimes J(B)$ (see [CuRe1], $\S 10 \mathrm{E}$ ) and for any $(A \otimes B)$-module $X$ we have $J(X)=X J(A \otimes B)$ (see [CuRe1] $\S 5 \mathrm{C}$ ). We get the general formula by using $J(M \otimes N)=J(M) \otimes N+M \otimes J(N)$ repeatedly.

The second formula is a little more technical to prove. Since $J^{k-1}(M \otimes N) / J^{k}(M \otimes$ $N)$ is semisimple we have that

$$
\left(J^{i}(M) \otimes J^{k-1-i}(N)\right) \cap \sum_{j=0, j \neq i}^{k-1} J^{j}(M) \otimes J^{k-1-j}(N) \subseteq J^{i}(M \otimes N)
$$

This implies

$$
\begin{aligned}
J^{k-1}( & M \otimes N) / J^{k}(M \otimes N) \cong\left(\sum_{i=0}^{k-1} J^{i}(M) \otimes J^{k-1-i}(N)+J^{k}(M \otimes N)\right) / J^{k}(M \otimes N) \\
& \cong \bigoplus_{i=0}^{k-1}\left(J^{i}(M) \otimes J^{k-1-i}(N)+J^{k}(M \otimes N)\right) / J^{k}(M \otimes N) \\
& \cong \bigoplus_{i=0}^{k-1}\left(J^{i}(M) \otimes J^{k-1-i}(N)\right) /\left(J^{i+1}(M) \otimes J^{k-1-i}(N)+J^{i}(M) \otimes J^{k-i}(N)\right) \\
& \cong \bigoplus_{i=0}^{k-1} J^{i}(M) / J^{i+1}(M) \otimes J^{k-1-i}(N) / J^{k-i}(N)
\end{aligned}
$$

We draw an easy consequence of this proposition:

Corollary 4.9. Let $L=\mathrm{Gl}_{e}(q)^{r} \times \mathrm{Gl}_{1}(q)^{s}$ with $s<e$ and $e>1$. Then the unique e-regular composition factor $D_{\text {reg }}(L)$ of $P_{S t}(L)$ belongs to the $r^{\text {th }}$ layer in the radical series where we count from top to bottom, starting with 0 .

Proof. The unique e-regular composition factor of $P_{S t}(L)$ has the form $D_{\text {reg }}(L)=$ $D_{\text {reg }}(e)^{\otimes r} \otimes D_{\text {reg }}(1)^{\otimes s}$. From Corollary 4.5 we know that $D_{r e g}(e)$ lies in $1^{\text {st }}$ layer of the radical series of $P_{S t}\left(\mathrm{Gl}_{e}(q)\right)$ and that $D_{\text {reg }}(1)$ lies in the $0^{\text {th }}$ and only layer of the radical series of $P_{S t}(1)$. So the second formula of Proposition 4.8 implies our result.

\subsection{The Endomorphism Ring of an Induced Module}

It is well known that for any irreducible cuspidal $F L$-module $C$ of a Levi subgroup $L$ of $G$, the endomorphism $\operatorname{ring} \operatorname{End}_{F G}\left(\mathrm{R}_{L}^{G} C\right)$ is a tensor product of Hecke algebras (see [Di1]). Now we look at a slightly different situation. 
Let $L \cong \mathrm{Gl}_{e}(q)^{r} \times \mathrm{Gl}_{1}(q)^{s}$ with $s<e$ be a standard Levi subgroup of $G$. We consider $\mathrm{R}_{L}^{G} P_{S t}(L)$, and we write henceforth $P:=P_{S t}(L)$ and $E:=\operatorname{End}_{F L}(P)$.

With Adjointness and Mackey decomposition we have

$$
\mathcal{E}:=\operatorname{End}_{F G}\left(\mathrm{R}_{L}^{G} P\right) \cong \operatorname{Hom}_{F L}\left(P, \mathrm{~T}_{L}^{G} \mathrm{R}_{L}^{G} P\right) \cong \bigoplus_{x \in \mathcal{D}_{L, L}} \operatorname{Hom}_{F L}\left(P, \mathrm{R}_{L^{x} \cap L}^{L} \mathrm{~T}_{L^{x} \cap L}^{L^{x}} P^{x}\right)
$$

Note that $\mathcal{E}=\operatorname{End}_{F G}\left(\mathrm{R}_{L}^{G} P\right)$ and $\operatorname{Hom}_{F L}\left(P, \mathrm{~T}_{L}^{G} \mathrm{R}_{L}^{G} P\right)$ are free right $E$-modules and that the isomorphism $\alpha: \mathcal{E} \rightarrow \bigoplus_{x \in \mathcal{D}_{L, L}} \operatorname{Hom}_{F L}\left(P, \mathrm{R}_{L^{x} \cap L}^{L} \mathrm{~T}_{L^{x} \cap L}^{L^{x}} P^{x}\right)$ is an isomorphism of $E$-modules. Now consider the $E$-submodule

$$
\tilde{H}:=\bigoplus_{\substack{x \in \mathcal{D}_{L, L} \\ L^{x}=L}} \operatorname{Hom}_{F L}\left(P, P^{x}\right) \subseteq \bigoplus_{x \in \mathcal{D}_{L, L}} \operatorname{Hom}_{F L}\left(P, \mathrm{R}_{L^{x} \cap L}^{L} \mathrm{~T}_{L^{x} \cap L}^{L^{x}} P^{x}\right)
$$

Denote by $H:=\alpha^{-1}(\tilde{H})$ the $E$-submodule of $\mathcal{E}$ corresponding to $\tilde{H}$ and by $W(L, P):=\left\{x \in \mathcal{D}_{L, L} \mid L^{x}=L\right\}$ a subgroup of the Weyl group which is called the ramification group of $L$.

First we need the structure of the ramification group:

Proposition 4.10. The ramification group is the subgroup of $S_{n}$ permuting diagonal blocks of the same size, i.e. $W(L, P) \cong S_{r} \times S_{s}$.

Proof. It is clear that each permutation of blocks of the same size satisfies $L^{x}=L$ and $P^{x} \cong P$. They are also in $\mathcal{D}_{L, L}$ since $x$ and $x^{-1}$ map positive roots in $W_{L}$ to positive roots. On the other hand any element with $L^{x}=L$ and $x \in \mathcal{D}_{L, L}$ must be a permutation of blocks of the same size since basic roots are mapped to basic roots in this case.

In the proof we actually use the following property of $L$.

Proposition 4.11. L is up to conjugation the unique minimal standard Levi subgroup of $G$ such such that $P_{S t}(G)$ is a direct summand of $\mathrm{R}_{L}^{G} P$. Thus for any subgroup $M \lessgtr L$ the Steinberg-PIM $P_{S t}(G)$ of $G$ is not a direct summand of $\mathrm{R}_{M}^{G} P_{S t}(M)$. This implies also that $P$ is not a direct summand of $\mathrm{R}_{M}^{L} P_{S t}(M)$ for any standard Levi subgroup $M \lessgtr L$.

Proof. Let $C$ be the cuspidal $F L$-module in the unipotent block of $L$, which exists by Theorem 2.5. The map $P_{S t}(L) \rightarrow C$ is a minimal projective cover of $C$. This can be lifted to $\mathrm{R}_{L}^{G} P_{S t}(L) \rightarrow \mathrm{R}_{L}^{G} C$. But the module $D_{F}\left(1,1^{n}\right)$ lies in the HC-series $S_{F}(L, C)$. So there exists a surjective homomorphism $f: \mathrm{R}_{L}^{G} C \rightarrow D_{F}\left(1,1^{n}\right)$. This shows that $\mathrm{R}_{L}^{G} P_{S t}(L)$ is a projective cover of $D_{F}\left(1,1^{n}\right)$. But $D_{F}\left(1,1^{n}\right)$ is also the head of $P_{S t}(G)$, so $P_{S t}(G)$ must be a direct summand of $\mathrm{R}_{L}^{G} P_{S t}(L)$. Now for any $M \lessgtr L$ we have $\mathrm{T}_{M}^{G} D_{F}\left(1,1^{n}\right)=0$ by the definition of the Harish-Chandra 
series. This implies $\operatorname{Hom}_{F G}\left(\mathrm{R}_{M}^{G} P_{S t}(M), D_{F}\left(1,1^{n}\right)\right)=0$. But as $D_{F}\left(1,1^{n}\right)$ is the head of $P_{S t}(G)$ there exists at least the projection map. Therefore $P_{S t}(G)$ cannot be a direct summand of $\mathrm{R}_{M}^{G} P_{S t}(M)$. This proves the minimality of $L$. Since $\mathrm{R}_{L}^{G} P_{S t}(L) \cong \mathrm{R}_{L^{x}}^{G} P_{S t}(L)^{x}$ all conjugates of $L$ have the same property.

Now suppose $\bar{L}$ is any minimal standard Levi subgroup such that $P_{S t}(G)$ is a direct summand of $\mathrm{R}_{\bar{L}}^{G} P_{S t}(\bar{L})$. Then $P_{S t}(L) \cong \mathrm{T}_{L}^{G} P_{S t}(G)$ is a direct summand of $\mathrm{T}_{L}^{G} \mathrm{R}_{\bar{L}}^{G} P_{S t}(\bar{L})$. This implies, by Mackey decomposition and the minimality of $L$, that there exists $x \in \mathcal{D}_{M, L}$ such that $\bar{L}^{x} \geq L$. By the minimality of $\bar{L}$ we have that $L$ and $\bar{L}$ are conjugate. This proves the uniqueness.

The last claim follows from transitivity of HC-induction (see Prop. 2.1). Assume that $P$ is a direct summand of $\mathrm{R}_{M}^{L} P_{S t}(M)$. Then $P_{S t}(G)$ is a direct summand of $\mathrm{R}_{M}^{G} P_{S t}(G)=\mathrm{R}_{L}^{G} \mathrm{R}_{M}^{L} P_{S t}(M)$ which is a contradiction to the minimality of $L$.

Now we can state the main theorem of this subsection:

Theorem 4.12. Let $\mathcal{B}=\left\{f_{1}, \ldots, f_{k}\right\}$ be an $F$-basis of $E=\operatorname{End}_{F L}(P)$. Then $H$ is a sub-algebra of $\mathcal{E}$ with a basis labelled $\left\{B_{w} f_{i} \mid w \in W(L, P), f_{i} \in \mathcal{B}\right\}$. Furthermore $H$ is a free E-module with basis $\left\{B_{w} \mid w \in W(L, P)\right\}$ and we have the following multiplication rule

$$
B_{v} f \cdot B_{w} g=\left\{\begin{array}{ll}
B_{v w}\left(f^{w} g\right) & \text { if } l(v w)=l(w)+1 \\
q^{-d^{2}} B_{v w}\left(f^{w} g\right)+\left(q^{d}-1\right) B_{w}\left(f^{w} g\right) & \text { if } l(v w)=l(w)-1
\end{array} .\right.
$$

Here $v$ denotes a basic transposition in $W(L, P)$ and $d$ is the size of the diagonal blocks of $L$ exchanged by $v$, which is either $e$ or 1 . The action of $w \in W(L, P)$ on $f \in E$ is given by the corresponding permutation of the entries in the tensor product, e.g.

$$
f^{w}\left(c_{1} \otimes \cdots \otimes c_{n}\right)=\left(f_{1} \otimes \cdots \otimes f_{n}\right)^{w}\left(c_{1} \otimes \cdots \otimes c_{n}\right):=f_{1 w}\left(c_{1}\right) \otimes \cdots \otimes f_{n w}\left(c_{n}\right)
$$

The proof mimics the structure of the proof used in [Di1] to calculate the multiplication in $\operatorname{End}_{F G}\left(\mathrm{R}_{L}^{G} C\right)$, where $C$ is a cuspidal $F L$-module. We use several lemmas and propositions to structure the proof.

First of all we have that for any $w \in W(L, P)$

$$
a_{w}: P \rightarrow P^{w} \quad c_{1} \otimes c_{2} \otimes \cdots \otimes c_{n} \mapsto c_{1 w^{-1}} \otimes c_{2 w^{-1}} \otimes \cdots \otimes c_{n w^{-1}}
$$

is an isomorphism of $F L$-modules since $P_{S t}(L)=P_{S t}\left(\mathrm{Gl}_{e}(q)\right)^{\otimes r} \otimes P_{S t}\left(\mathrm{Gl}_{1}(q)\right)^{\otimes s}$. The second important map is

$$
B_{w, P^{w}}: \mathrm{R}_{T}^{G} P^{w} \rightarrow \mathrm{R}_{T}^{G} P \quad \sum_{g \in P_{L} / G} m_{g} \underset{F P_{L}}{\otimes} g \mapsto \sum_{g \in P_{L} / G} m_{g} \underset{F P_{L}}{\otimes} w \hat{U}_{w^{-1}}^{-} g=\sum_{g \in P_{L} / G} m_{g} \underset{F P_{L}}{\otimes} w \hat{U} g
$$


where $m_{g} \in P^{w}$ for all $g$ and $\hat{U}=\frac{1}{|U|} \sum_{u \in U} u$. It was shown in [DiDu], that this map is a natural equivalence of functors. This implies immediately that

$$
\left(a_{x} \otimes i d\right) \circ B_{y, P^{y}}=B_{y, P^{x y}} \circ\left(a_{x} \otimes i d\right) \quad x, y \in W(L, P)
$$

The following lemma will be needed for the first part of the multiplication rule:

Lemma 4.13. Let $x, y \in W(L, P)$ such that $l(x y)=l(x)+l(y)$. Then

$$
B_{x, P^{x}} \circ B_{y, P^{x y}}=B_{x y, P^{x y}} .
$$

Proof. This is Proposition 10.2.2 in [Carter].

The following proposition establishes a basis for $H$ with the maps introduced before.

Proposition 4.14. $\left\{B_{w, P w} \circ\left(a_{w} \otimes i d\right) \circ(f \otimes i d) \mid w \in W(L, P), f \in \mathcal{B}\right\}$ is a basis of $H$. We write $B_{w}:=B_{w, P w} \circ\left(a_{w} \otimes i d\right)$. Furthermore $\left\{B_{w} \mid w \in W(L, P)\right\}$ is an $E$-basis of $H$.

Proof. We use adjointness and the Mackey formula to get a basis:

$$
\begin{aligned}
& \operatorname{End}_{F G}\left(\mathrm{R}_{L}^{G} P\right) \cong \operatorname{Hom}_{F L}\left(P, \mathrm{~T}_{T}^{G} \mathrm{R}_{T}^{G} P\right) \cong \bigoplus_{w \in \mathcal{D}_{L, L}} \operatorname{Hom}_{F L}\left(P, \mathrm{R}_{L^{w} \cap L}^{L} \mathrm{~T}_{L^{w} \cap L}^{L^{w}} P^{w}\right) \\
& \cong \underbrace{\bigoplus_{w \in W(L, P)} \operatorname{Hom}_{F L}\left(P, P^{w}\right)}_{=\tilde{H}} \oplus \bigoplus_{w \in \mathcal{D}_{L, L} \backslash W(L, P)} \operatorname{Hom}_{F L}\left(P, \mathrm{R}_{L^{w} \cap L}^{L} P_{S t}\left(L \cap L^{w^{-1}}\right)^{w}\right)
\end{aligned}
$$

Now $\left\{a_{w} \circ f \mid f \in \mathcal{B}\right\}$ is a basis of $\operatorname{Hom}_{F L}\left(P, P^{w}\right)$. Following the isomorphisms backwards we get that $\left\{B_{w, P w} \circ\left(a_{w} \otimes i d\right) \circ(f \otimes i d) \mid w \in W(L, P), f \in \mathcal{B}\right\}$ is a basis of $H$. Since all these isomorphisms are $E$-module isomorphisms we get that $\left\{B_{w} \mid w \in W(L, P)\right\}$ is an $E$-basis of $H$.

Henceforth we will shorten $B_{w} \circ(f \otimes i d)$ to $B_{w} f$ when this leaves no ambiguity. The next step is the first half of the multiplication rule:

Proposition 4.15. Let $w \in W(L, P)$ be arbitrary and let $v \in W(L, P)$ be a basic transposition (exchanging diagonal blocks next to each other) such that $l(v w)=$ $l(w)+1$. Then

$$
B_{v} f \circ B_{w} g=B_{v w}\left(f^{w} g\right)
$$


Proof. Using Lemma 4.13 and the natural equivalence of $B_{w, P^{w}}$ we get:

$$
\begin{aligned}
B_{v} f \circ B_{w} g & =B_{v, P^{v}} \circ\left(a_{v} \otimes i d\right) \circ(f \otimes i d) \circ B_{w, P^{w}} \circ\left(a_{w} \otimes i d\right) \circ(g \otimes i d) \\
& =B_{v, P^{v}} \circ B_{w, P^{v w}} \circ\left(a_{v} f a_{w} g \otimes i d\right) \\
& =B_{v w, P^{v w}} \circ\left(a_{v w} \otimes i d\right) \circ\left(f^{w} g \otimes i d\right) \\
& =B_{v w}\left(f^{w} g\right)
\end{aligned}
$$

For the second case we will consider a special case first. We take $n=2 k$ with $k=1$ or $k=e$ and we take $L=\mathrm{Gl}_{k}(q)^{2}$. Proposition 4.14 tells us that $\left\{B_{w} f \mid\right.$ $w \in\{1, v\}, f \in \mathcal{B}\}$ is a basis of $H$, with $v$ standing for the exchange of the two blocks of $L$. The only relation to work out is the one for $B_{v}^{2}$. Here we have the following:

Proposition 4.16. Assume that $L$ has the properties of Proposition 4.11 i.e. $L$ is minimal such that $P_{S t}(G)$ is a direct summand of $\mathrm{R}_{L}^{G} P$. With the notation above, we have $B_{v}^{2}=1 a+B_{v} b$, with $a, b \in E$.

Proof. Since $P_{S t}(G)$ is a direct summand of $\mathrm{R}_{L}^{G} P$ by assumption the projection $\pi: \mathrm{R}_{L}^{G} P \rightarrow P_{S t}(G) \leq \mathrm{R}_{L}^{G} P$ is an element of $\operatorname{End}_{F L}\left(\mathrm{R}_{L}^{G} P\right)$. Since $\mathrm{T}_{L}^{G} P_{S t}(G) \cong P$ we have that the image of $\pi$ under the Frobenius reciprocity is contained in the component $P \oplus P^{v}$, since no other component contains a copy of $P$ since they are all induced from smaller Levi subgroups. So we have $\pi \in H$. This implies:

$$
\pi=1 g+B_{v} h \text { and } \pi^{2}=1 g^{2}+B_{v}\left(g^{v} h+h g\right)+B_{v}^{2} h^{v} h=1 g+B_{v} h=\pi
$$

where $g, h \in E$. Now we assume $B_{v}^{2}=1 a+B_{v} b+x$, where $a, b \in E$ and $x$ belongs to an $F$-space complement of $H$ in $\operatorname{End}_{F L}\left(\mathrm{R}_{L}^{G} P\right)$. Comparing coefficients in (1) we get $x h^{v} h=0$. We have to establish $x=0$. To achieve this, we show that $h$ is invertible. So assume that $h$ is not invertible. Now we remark that $E$, being the endomorphism ring of a PIM, is a local ring. So every non-invertible element is actually nilpotent and $E=F \oplus J(E)$ as $F$-space. Since $\pi$ is an idempotent, it cannot be nilpotent, therefore $g=\alpha 1_{E}+\bar{g}$ has to be invertible, with $\alpha \in F^{*}$ and $\bar{g} \in E$ nilpotent. Comparing coefficients of $1_{H}=B_{1}$ we get

$$
\alpha^{2} 1_{E}+\underbrace{2 \alpha \bar{g}+\bar{g}^{2}+h^{v} h a}_{\in J(E)}=\alpha 1_{E}+\bar{g}
$$

and we conclude that $\alpha=1$. But now we compare the coefficients of $B_{v}$ and get

$$
\underbrace{2 h}_{\in J^{k}(E)}+\underbrace{\bar{g}^{v} h+h \bar{g}+h^{v} h b}_{\in J^{k+1}(E)}=\underbrace{h}_{\in J^{k}(E)}
$$


where $k$ is chosen such that $h \in J^{k}(E)$ but $h \notin J^{k+1}(E)$. The equation remains true modulo $J^{k+1}(E)$ and so we conclude $h=0$ modulo $J^{k+1}(E)$. By the choice of $k$ this is only possible if $h=0$. But this implies, that $\pi$ is invertible, and therefore $\pi=i d$. But this is impossible. Since the equation $\mathrm{R}_{L}^{G} P \cong P_{S t}(G)$ would also be true in characteristic 0 we can look at the ordinary Steinberg module of $L$, which is $S_{K}\left(1,1^{k}\right) \otimes S_{K}\left(1,1^{k}\right)$. Its induction to $G$ is governed by the Littlewood-Richardson rule, so we see that it contains $S_{K}\left(1,\left(2^{k}\right)\right)$ for example, which does not lie in $P_{S t}(G)$. So we must conclude that $h$ is invertible and the proposition is proved.

Now we take again $L=\mathrm{Gl}_{e}(q)^{r} \times \mathrm{Gl}_{1}(q)$.

Lemma 4.17. Let $P_{S t}(G)$ be a direct summand of $\mathrm{R}_{L}^{G} P_{S t}(L)$. Then for any Levi subgroup $L \leq M \leq G, P_{S t}(M)$ is a direct summand of $\mathrm{R}_{L}^{M} P$.

Proof. $P_{S t}(G)$ is the projective cover of the Steinberg module $S_{F}\left(1,1^{n}\right)$, therefore there exists a nonzero homomorphism in $\operatorname{Hom}_{F G}\left(P_{S t}(G), S_{F}\left(1,1^{n}\right)\right)$. Since $P_{S t}(G)$ is a direct summand of $\mathrm{R}_{L}^{G} P$ this induces a nonzero homomorphism $\mathrm{R}_{L}^{G} P \rightarrow$ $S_{F}\left(1,1^{n}\right)$. By adjointness of functors we have

$$
0 \neq \operatorname{Hom}_{F G}\left(\mathrm{R}_{L}^{G} P, S_{F}\left(1,1^{n}\right)\right) \cong \operatorname{Hom}_{F M}\left(\mathrm{R}_{L}^{M} P, \mathrm{~T}_{M}^{G} S_{F}\left(1,1^{n}\right)\right)
$$

But $\mathrm{T}_{M}^{G} S_{F}\left(1,1^{n}\right)$ is the Steinberg module of $F M$ and $\mathrm{R}_{L}^{M} P$ is projective. Since $P_{S t}(M)$ is the minimal projective cover of the Steinberg module it must be a direct summand of $\mathrm{R}_{L}^{M} P$, as claimed.

Now we have gathered all the ingredients for the second part of the multiplication rules:

Proposition 4.18. Let $w \in W(L, P)$ be arbitrary and let $v \in W(L, P)$ be a basic transposition such that $l(v w)=l(w)-1$. Then

$$
B_{v} \circ B_{w}=B_{v w} a+B_{w} b
$$

with $a, b \in E$.

Proof. Since $l(v w)=l(w)-1$ this implies, that $w=v w^{\prime}$ with $l(w)=l\left(w^{\prime}\right)+1$. Therefore $B_{w}=B_{v} \circ B_{w^{\prime}}$ by Proposition 4.15. So we can assume $w=v$ and just look at $B_{v}^{2}$. Now let $M:=L(v)$ be the smallest Levi subgroup of $G$ containing $L$ and $v$. From Lemma 4.17 we know that $P_{S t}(M)$ is a direct summand of $\mathrm{R}_{L}^{M} P$. Just looking at the two blocks exchanged by $v$ we get by Proposition 4.16 that on $\mathrm{R}_{L}^{M} P$ we have $B_{v}^{2}=1 a+B_{v} b$. But Harish-Chandra induction from $M$ to $G$ then gives the same on $\mathrm{R}_{L}^{G} P$. 
We now want to calculate the coefficients $a$ and $b$ in the formula of Proposition 4.18. Note that by the proof of that theorem these coefficients do not depend on $w$, but only on $v$ and are the same as those of Proposition 4.16. We let both sides of the equation $(2)$ act on $(m \otimes 1)$ with $m \in P$ arbitrary. This gives:

$$
m \otimes v \hat{U}_{v}^{-} v \hat{U}_{v}^{-}=a(m) \otimes 1+a_{v} \circ b(m) \otimes v \hat{U}_{v}^{-}
$$

Now we use the unique decomposition of $P \underset{F P_{L}}{\otimes} F G$ as $\sum_{g \in P_{L} / G} P \underset{F P_{L}}{\otimes} g$ and compare coefficients for $g=1$. Since $v U_{v}^{-} v \cap P_{L}=\{1\}$ and $v \notin P_{L}$ we have

$$
m \otimes \frac{1}{\left|U_{v}^{-}\right|} \hat{U}_{v}^{-}=a(m) \otimes 1
$$

Furthermore, $u \in U_{v}^{-} \subset U_{L}$ acts trivially on the $F L$-module $P$, so $a$ acts on $P$ as $\frac{1}{\left|U_{v}^{-}\right|}=q^{-d^{2}}$ where $d$ is the size of the blocks exchanged by $v$.

For $b$ we use the same technique and let both both sides of the equation act on $m \otimes v$. We get

$$
m \otimes v \hat{U}_{v}^{-} v \hat{U}_{v}^{-} v=a(m) \otimes v+a_{v} \circ b(m) \otimes v \hat{U}_{v}^{-} v
$$

Comparing coefficients again we get the following condition:

$$
b(m) \otimes 1=\frac{1}{\mid U_{v}^{-\mid}} \sum_{\substack{u, u^{\prime} \in U_{v}^{-} \\ v u v u^{\prime} v \in P_{L}}} a_{v}^{-1}(m) v u v u^{\prime} v \otimes 1
$$

Before we continue, we need a result from ordinary representation theory of finite groups:

Lemma 4.19. Let $C, C^{\prime}$ be non-isomorphic irreducible $K G$-modules and let $T$ : $G \rightarrow \mathrm{Gl}(C)$ and $T^{\prime}: G \rightarrow \mathrm{Gl}\left(C^{\prime}\right)$ be the corresponding matrix representations. Then:

$$
\sum_{g \in G} v_{1} T\left(g^{-1}\right) \otimes v_{2} T^{\prime}(g)=0 \text { and } \sum_{g \in G} v_{1} T\left(g^{-1}\right) \otimes v_{2} T(g)=\frac{|G|}{\operatorname{dim} C} v_{2} \otimes v_{1}
$$

for any $v_{1} \otimes v_{2} \in C \otimes C^{\prime}$.

Proof. It is enough to prove the statement for a basis $\left\{e_{i} \otimes e_{j} \mid 1 \leq i, j \leq \operatorname{dim} C\right\}$ where $\left\{e_{1}, \ldots, e_{\operatorname{dim} C}\right\}$ is the standard basis of $C$. Now we use orthogonality relations for representations (see e.g. [Hu], §V.5, Theorem 5.7):

$$
\sum_{g \in G} T_{i j}\left(g^{-1}\right) T_{k l}^{\prime}(g)=0 \quad \text { and } \quad \sum_{g \in G} T_{i j}\left(g^{-1}\right) T_{k l}(g)=\delta_{j k} \delta_{i l} \frac{|G|}{\operatorname{dim} C}
$$


Therefore

$$
\begin{aligned}
\sum_{g \in G} e_{i} T\left(g^{-1}\right) \otimes e_{k} T^{\prime}(g) & =\sum_{g \in G} \sum_{j, l} e_{j} T_{i j}\left(g^{-1}\right) \otimes e_{l} T_{k l}^{\prime}(g) \\
& =\sum_{j, l} e_{j} \otimes e_{l}\left(\sum_{g \in G} T_{i j}\left(g^{-1}\right) T_{k l}^{\prime}(g)\right)=0
\end{aligned}
$$

and

$$
\begin{aligned}
\sum_{g \in G} e_{i} T\left(g^{-1}\right) \otimes e_{k} T(g) & =\sum_{g \in G} \sum_{j, l} e_{j} T_{i j}\left(g^{-1}\right) \otimes e_{l} T_{k l}(g) \\
& =\sum_{j, l} e_{j} \otimes e_{l}\left(\sum_{g \in G} T_{i j}\left(g^{-1}\right) T_{k l}(g)\right) \\
& =\frac{|G|}{\operatorname{dim} C} \sum_{j, l}\left(e_{j} \otimes e_{l}\right) \delta_{j k} \delta_{i l} \\
& =\frac{|G|}{\operatorname{dim} C} e_{k} \otimes e_{i}
\end{aligned}
$$

To simplify notation we assume that $v$ exchanges the first and the second block. If $v$ would exchange any two other blocks the result is the same. This leads to

$$
v u v u^{\prime} v=\left(\begin{array}{ccccc}
u^{\prime} & 1 & & & \\
1+u u^{\prime} & u & & & \\
& & 1 & & \\
& & & \ddots & \\
& & & & 1
\end{array}\right)
$$

Recall from equation (3) that $u, u^{\prime} \in U_{v}^{-}$are $d \times d$ matrices. So 1 really is the appropriate identity matrices. From (3) we also have the condition $v u v u^{\prime} v \in P_{L}$ which implies $1+u u^{\prime}=0$, so $u^{\prime}=-u^{-1} \in \mathrm{Gl}_{d}(q)$. Now suppose $m=\left(c_{1} \otimes \cdots \otimes\right.$ $\left.c_{n}\right) \in P$. Observe that $\left|U_{v}^{-}\right|=q^{d^{2}}$ so we get

$$
\begin{aligned}
b\left(c_{1} \otimes \cdots \otimes c_{n}\right) & =q^{-d^{2}} \sum_{u \in \mathrm{Gl}_{d}(q)} c_{2}\left(-u^{-1}\right) \otimes c_{1} u \otimes \cdots \otimes c_{n} \\
& =-q^{-d^{2}} \sum_{u \in \mathrm{Gl}_{d}(q)} c_{2} u^{-1} \otimes c_{1} u \otimes \cdots \otimes c_{n}
\end{aligned}
$$

To actually calculate $b$ we need to lift the equation to $O G$ and then tensor with $K$ to get a problem in characteristic zero. This is possible since Harish-Chandra induction and restriction commute with this and $P$ is a projective module. Since 
$P$ is a direct summand of the Gelfand-Graev module each ordinary constituent has multiplicity 1 . So we have two cases to consider: $c_{1} \otimes c_{2} \in C \otimes C^{\prime}$ with $C \neq C^{\prime}$ and $c_{1} \otimes c_{2} \in C \otimes C$. Both cases are dealt with by Lemma 4.19. This gives us:

$$
b\left(c_{1} \otimes \cdots \otimes c_{n}\right)= \begin{cases}0 & \text { if } C \not C^{\prime} \\ -q^{-d^{2}} \frac{\left|\mathrm{Gl}_{d}(q)\right|}{\operatorname{dim}_{K} C}\left(c_{1} \otimes \cdots \otimes c_{n}\right) & \text { otherwise }\end{cases}
$$

If $d=1$, then the Steinberg-PIM $P_{S t}\left(\mathrm{Gl}_{d}(q)\right)$ is equal to the Steinberg module, since $\ell$ does not divide the order of $\mathrm{Gl}_{d}(q)$ in this case. The dimension of the ordinary Steinberg module is $q^{d(d-1) / 2}$ (see [Carter],§6.4). This implies

$$
\frac{\left|\mathrm{Gl}_{d}(q)\right|}{\operatorname{dim}_{K} C}=(q-1)=\left(q^{d}-1\right)
$$

In the case of $d=e$ we have, that each ordinary module in $P_{S t}\left(\mathrm{Gl}_{d}(q)\right)$ has degree $\left|\mathrm{Gl}_{d}(q): C_{\mathrm{Gl}_{d}(q)}(s)\right|_{p^{\prime}}\left|C_{\mathrm{Gl}_{d}(q)}(s)\right|_{p}$ where $s$ is the semisimple $\ell$-element describing the module (see [Carter], $\S 8.4$ ). All modules other than the Steinberg module contain at least one factor $\mathrm{Gl}_{1}\left(q^{e}\right)$ in $C_{\mathrm{Gl}_{d}(q)}(s)$. Therefore in all cases (including the Steinberg module) $\left(q^{e}-1\right)$ divides $\frac{\left|\mathrm{Gl}_{d}(q)\right|}{\operatorname{dim}_{k} C}$ which in turn implies that the factor is $0=\left(q^{e}-1\right)$ over $F$. This proves the second part of the multiplication rule.

We get an easy corollary to Theorem 4.12:

Corollary 4.20. The sub-algebra $H$ in $\mathcal{E}$ has the form $H \cong\left(F S_{r} \otimes \mathcal{H}_{q, F}\left(S_{s}\right)\right) \otimes_{F} E$ and its radical has the form $J(H) \cong\left(F S_{r} \otimes \mathcal{H}_{q, F}\left(S_{s}\right)\right) \otimes J(E)$. The quotient is isomorphic to $F S_{r} \otimes \mathcal{H}_{q, F}\left(S_{s}\right)$, which is semisimple.

Proof. The form of the algebra is just a restatement of Theorem 4.12. Note that because of $s<e<\ell$ the Hecke algebra $\mathcal{H}_{q, F}\left(S_{s}\right)$ is semisimple and actually isomorphic to $F S_{s}$. From general theory (see e.g. [CuRe1], Theorem 10.38) we know that the radical has the form $J\left(F S_{r} \otimes F S_{s}\right) \otimes E+\left(F S_{r} \otimes F S_{s}\right) \otimes J(E)$. But $F S_{r} \otimes F S_{s}$ is semisimple because $e \ell>n$, so $\ell>n / e \geq r$. So $J\left(F S_{r} \otimes F S_{s}\right)=0$ and the rest follows.

In the next step, we decompose $\mathrm{R}_{L}^{G} P_{S t}(L)$ into PIMs:

Proposition 4.21. Let $L=\mathrm{Gl}_{e}(q)^{r} \times \mathrm{Gl}_{1}(q)^{s}$ with $s<e$. Then

$$
\mathrm{R}_{L}^{G} P_{S t}(L) \cong \bigoplus_{\substack{\rho \vdash r, \alpha \vdash s \\ \lambda^{\prime}=\alpha^{\prime}+e \rho^{\prime}}} m_{\lambda} P(\lambda)
$$

where $P(\lambda)$ is the projective indecomposable module with head $D_{F}(1, \lambda)=:[\lambda]$ and $m_{\lambda}$ denotes the multiplicity of $[\lambda]$ in $\mathrm{R}_{L}^{G} C$. Here $C$ is the cuspidal FL-module $D_{F}\left(1,1^{e}\right)^{\otimes r} \otimes D_{F}(1,1)^{\otimes s}$. 
Proof. We know that $\mathrm{R}_{L}^{G} P_{S t}(L)$ is a projective cover of $\mathrm{R}_{L}^{G} C$, since $C$ is the head of $P_{S t}(L)$. The multiplicity comes from the following calculation:

$$
\begin{aligned}
& \sharp P(\lambda) \text { direct summand of } \mathrm{R}_{L}^{G} P_{S t}(L)=\operatorname{dim}_{F} \operatorname{Hom}_{F G}\left(\mathrm{R}_{L}^{G} P_{S t}(L),[\lambda]\right) \\
& \quad=\operatorname{dim}_{F} \operatorname{Hom}_{F L}\left(P_{S t}(L), \mathrm{T}_{L}^{G}[\lambda]\right)=\operatorname{dim}_{F} \operatorname{Hom}_{F L}\left(C, \mathrm{~T}_{L}^{G}[\lambda]\right) \\
& \quad=\operatorname{dim}_{F} \operatorname{Hom}_{F G}\left(\mathrm{R}_{L}^{G} C,[\lambda]\right)=m_{\lambda}
\end{aligned}
$$

In the second line we use that $\mathrm{T}_{L}^{G}[\lambda]$ is semisimple (Theorem 3.7) and in the third line, that $\mathrm{R}_{L}^{G} C$ is also semisimple, since $\operatorname{End}_{F G}\left(\mathrm{R}_{L}^{G} C\right) \cong \mathcal{H}_{q^{e}, F}\left(S_{r}\right) \otimes \mathcal{H}_{q, F}\left(S_{s}\right) \cong$ $F S_{r} \otimes F S_{s}$ is semisimple.

Now suppose $P(\lambda)$, for some $\lambda \vdash n$, is a direct summand of $\mathrm{R}_{L}^{G} P_{S t}(L)$. Then as above $C$ has to be a simple constituent of the semisimple module $\mathrm{T}_{L}^{G}[\lambda]$. This in turn implies $\operatorname{Hom}_{F L}\left(\mathrm{~T}_{L}^{G} P(\lambda), C\right) \neq 0$. Then $\operatorname{Hom}_{F G}\left(P(\lambda), \mathrm{R}_{L}^{G} C\right) \neq 0$ and since $\mathrm{R}_{L}^{G} C$ is semisimple, this means that $[\lambda]$ belongs to the Harish-Chandra series $S(L, C)$. So $\lambda \vdash n$ satisfies $\lambda^{\prime}=\alpha^{\prime}+e \rho^{\prime}$ with $\rho \vdash r$ and $\alpha \vdash s$.

Now we identify the PIMs of $H$ :

Proposition 4.22. Let $\bar{e}_{1}+\cdots+\bar{e}_{k}=1 \in H / J(H)$ be a decomposition into pairwise orthogonal, primitive idempotents. Then there exist $e_{i} \in H$ such that $e_{i}+J(H)=\bar{e}_{i}$ and $e_{1}+\cdots+e_{k}=1 \in H \subseteq \mathcal{E}$ is a decomposition into pairwise orthogonal primitive idempotents in $\mathcal{E}$.

Proof. We know by Corollary 4.20 that $H \cong H / J(H) \otimes E$. We take $e_{i}:=\bar{e}_{i} \otimes 1$. From the rules of multiplication and addition it is clear that the $e_{i}$ are pairwise orthogonal idempotents. They have to be primitive, since the number of primitive idempotents is the same in $H / J(H) \cong \operatorname{End}_{F G}\left(\mathrm{R}_{L}^{G} C\right)$ and in $\mathcal{E}$, which was shown in Proposition 4.21.

Before we finish with the structure of the radical series of $H$, we will use the previous proposition to draw some facts about the structure of $\mathcal{E}$ :

Proposition 4.23. We have

$$
\mathcal{E}=H+J(\mathcal{E})
$$

where $J(\mathcal{E})$ denotes the usual Jacobson radical of $\mathcal{E}$. Furthermore, every simple $\mathcal{E}$-module is simple as $H$-module.

Proof. From Proposition 4.22 we know that every primitive idempotent of $\mathcal{E}$ lies in $H$. Therefore $\mathcal{E} / J(\mathcal{E}) \cong H / J(H)$ which proves the first claim. The second one is an easy consequence of $\mathcal{E}=H+J(\mathcal{E})$ since $J(\mathcal{E})$ acts as zero on any simple $\mathcal{E}$ module $X$, so $H$ and $\mathcal{E}$ act the same on $X$ and $X$ can only be simple as $\mathcal{E}$-module if it is simple as $H$-module. 
Let $f$ be any of the primitive idempotents of $H$. Then the radical of $f H$ is $f H \cdot J(H)=f J(H)$ since $H$ is a semi-local ring (see [CuRe1], Prop. 5.29). So we inductively get (by Cor. 4.20):

$$
J^{k}(f H)=f J^{k}(H) \cong\left(\bar{f}\left(F S_{r} \otimes F S_{s}\right)\right) \otimes J^{k}(E)
$$

To calculate the radical series of $f H$ we have to decompose $J^{k}(f H) / J^{k+1}(f H)$. The factor is

$$
J^{k}(f H) / J^{k+1}(f H) \cong\left(\bar{f}\left(F S_{r} \otimes F S_{s}\right)\right) \otimes J^{k}(E) / J^{k+1}(E)
$$

Since $J(E)$ acts as zero, this is a semisimple $F S_{r} \otimes F S_{s}$-module. The irreducible constituents of $F S_{r} \otimes F S_{s}$ are tensor products of Specht modules $S^{\lambda_{1}} \otimes S^{\lambda_{2}}$ labelled by bipartitions $\left(\lambda_{1}, \lambda_{2}\right)$, where $\lambda_{1} \vdash r$ and $\lambda_{2} \vdash s$. The projective indecomposable $H$-modules are also labelled by such bipartitions.

To formulate the next result, which gives the decomposition of the $k^{\text {th }}$ radical layer we need some notation. Denote by $M^{\left(\lambda_{1}, \lambda_{2}\right)}$ the permutation module of $F S_{r} \otimes F S_{s}$ belonging to the bipartition $\left(\lambda_{1}, \lambda_{2}\right)$. For any $\rho \vdash r$ we write $\operatorname{comp}(k, d, \rho)$ for the number of compositions of $k$ of the form $\left(x_{1}^{\rho_{1}}, x_{2}^{\rho_{2}}, \ldots, x_{j}^{\rho_{j}}\right)$ where the $x_{i}$ are pairwise different integers with $0 \leq x_{i} \leq d-1$.

Theorem 4.24. Let $\left(\lambda_{1}, \lambda_{2}\right)$ and $\left(\mu_{1}, \mu_{2}\right)$ be bipartitions of $(r, s)$. Counting the radical layers from top to bottom (and starting with the $0^{\text {th }}$ layer) we obtain the following formula for the number of composition factors of type $S^{\lambda_{1}} \otimes S^{\lambda_{2}}$ in the $k^{\text {th }}$ layer of the PIM labelled by $\left(\mu_{1}, \mu_{2}\right)$ of the algebra $H$ :

$$
\sum_{\rho \vdash r} \operatorname{comp}(k, d, \rho) \cdot \operatorname{dim}_{F} \operatorname{Hom}_{F S_{r} \otimes F S_{s}}\left(S^{\lambda_{1}} \otimes S^{\lambda_{2}},\left(S^{\mu_{1}} \otimes S^{\mu_{2}}\right) \otimes M^{(\rho,(s))}\right)
$$

Here $d$ equals the length of the Loewy series of $P_{S t}\left(\mathrm{Gl}_{e}(q)\right)$.

Proof. We start by looking at $J^{k}(E) / J^{k+1}(E)$. Denote by $\tilde{E}$ the endomorphism $\operatorname{ring} \tilde{E}:=\operatorname{End}_{F \mathrm{Gl}_{e}(q)}\left(P_{S t}\left(\mathrm{Gl}_{e}(q)\right)\right)$. By the formula in Proposition 4.8 we know that as $F$-vector spaces we have

$$
\begin{aligned}
& J^{k}(E) / J^{k+1}(E) \cong \bigoplus_{\substack{0 \leq i_{1}, \ldots, i_{r} \leq d-1 \\
i_{1}+\cdots+i_{r}=k}} J^{i_{1}}(\tilde{E}) / J^{i_{1}+1}(\tilde{E}) \otimes \cdots \otimes J^{i_{r}}(\tilde{E}) / J^{i_{r}+1}(\tilde{E}) \otimes F^{\otimes s} \\
& \cong \bigoplus_{\substack{0 \leq i_{1}, \ldots, i_{r} \leq d-1 \\
i_{1}+\cdots+i_{r}=k}} F \cong \bigoplus_{\rho \vdash r} \bigoplus_{\substack{\left(i_{1}, \ldots, i_{r}\right)=\left(x_{1}^{\rho_{1}}, \ldots x_{j}^{\rho_{j}}\right) \\
i_{1}+\cdots+i_{r}=k}} M^{\rho} \\
& \cong \bigoplus_{\rho \vdash r} \operatorname{comp}(k, d, \rho) M^{(\rho,(s))}
\end{aligned}
$$


The last expression also respects the action of $F S_{r} \otimes F S_{s}$ on $J^{k}(E) / J^{k+1}(E)$. Therefore

$$
J^{k}(f H) / J^{k+1}(f H) \cong \bigoplus_{\rho \vdash r} \operatorname{comp}(k, d, \rho)\left(S^{\mu_{1}} \otimes S^{\mu_{2}}\right) \otimes M^{(\rho,(s))}
$$

Since everything is semisimple, counting the dimension of the Hom space is the same as counting the number of composition factors. This proves the theorem.

\subsection{The Special Case $e=1$}

We want to look at the special case $e=1$, because here we can get the Loewy series of all PIMs in the unipotent block, not just the series of the Steinberg PIM. The reason is that we have a convenient projective generator and therefore a complete knowledge of the endomorphism ring of this generator. Note that the condition $e \ell>n$ specialises to $\ell>n$ in this case.

Proposition 4.25. Let $1_{T}$ be the trivial module of FT and denote by $P_{S t}(T)=$ $P\left(1_{T}\right)$ a projective cover of $1_{T}$. Then $\mathrm{R}_{T}^{G} P\left(1_{T}\right)$ is a pro-generator for the unipotent block.

Proof. The endomorphism ring $\operatorname{End}_{F G}\left(\mathrm{R}_{T}^{G} 1_{T}\right)$ of $\mathrm{R}_{T}^{G} 1_{T}$ is the Hecke algebra $\mathcal{H}_{F, q}\left(S_{n}\right)$ (see [Di1]) and since $\ell$ does not divide $n$ it is semisimple. Therefore $\mathrm{R}_{T}^{G} 1_{T}$ is a direct sum of simple modules. Since all simple $F G$-modules are contained in the Harish-Chandra series $S_{F}\left(T, 1_{T}\right)$ the lifting $\mathrm{R}_{T}^{G}(f): \mathrm{R}_{T}^{G} P\left(1_{T}\right) \rightarrow \mathrm{R}_{T}^{G} 1_{T}$ of $f: P\left(1_{T}\right) \rightarrow 1_{T}$ is a projective presentation of the block and $\mathrm{R}_{T}^{G} P\left(1_{T}\right)$ therefore a pro-generator.

Since there exist no Levi subgroups $L<T$ we immediately get from the definition, that in this special case $H=\operatorname{End}_{F G}\left(\mathrm{R}_{T}^{G} P\left(1_{T}\right)\right)$ and furthermore $H \cong F S_{n}$. This shows, that we can use Morita equivalence (see [CuRe1], §3D) to calculate the Loewy series of the PIMs from the Loewy series of $H$ as described in theorem 4.24. The only thing we need to know is the correspondence between the labels of the irreducibles. Not surprisingly we have:

Proposition 4.26. Under the correspondence of the Morita equivalence between $\mathrm{R}_{T}^{G} P\left(1_{T}\right)$ and $H$ the irreducible $D_{F}(1, \lambda)$ corresponds to the irreducible Specht module $S^{\lambda}$.

Proof. This is a special case of Proposition 4.36.

We restate theorem 4.24 in the language of $F G$-modules: 
Proposition 4.27. Let $\lambda, \mu$ be partitions of $n$. Counting the radical layers from top to bottom we get the following formula for the number of composition factors of $P^{\mu}$ of type $S_{F}(1, \lambda)$ in the $k^{\text {th }}$ radical layer:

$$
\sum_{\rho \vdash n} \operatorname{comp}(k, d, \rho) \cdot \operatorname{dim}_{F}\left(\operatorname{Hom}_{F S_{n}}\left(S^{\lambda}, S^{\mu} \otimes M^{\rho}\right)\right)
$$

where $d=\left|\left(\mathrm{GF}(q)^{*}\right)_{\ell}\right|$.

\subsection{Main Theorem}

We want to put the gathered knowledge to use and state a relation between the radical series of $P_{S t}(L)$ and the radical series of $P_{S t}(G)$ in almost all cases of Abelian defect. We assume $e>1$ since the proofs given here do not work in the special case $e=1$.

Theorem 4.28. Let $L=\mathrm{Gl}_{e}(q)^{r} \times \mathrm{Gl}_{1}(q)^{s}$ with $s<e$ and $e>1$. Then for any standard Levi subgroup $L \leq M \leq G$ and any non-negative integer $i$ we have:

$$
\mathrm{T}_{M}^{G} J^{i}\left(P_{S t}(G)\right)=J^{i}\left(P_{S t}(M)\right)
$$

We start by showing some lemmas. Let $L=\mathrm{Gl}_{e}(q)^{r} \times \mathrm{Gl}_{1}(q)^{s}$ as above.

Lemma 4.29. Let $M \leq G$ be a standard Levi subgroup and let $X \leq P_{S t}(G)$. Then for all non-negative integers $i$ we have $J^{i}\left(\mathrm{~T}_{M}^{G} X\right) \subseteq \mathrm{T}_{M}^{G} J^{i}(X)$.

Proof. Since HC-restriction is exact, we have the following short exact sequence:

$$
0 \rightarrow \mathrm{T}_{M}^{G} J(X) \rightarrow \mathrm{T}_{M}^{G} X \rightarrow \mathrm{T}_{M}^{G} \mathrm{hd}(X) \rightarrow 0
$$

By Theorem 3.7 we know, that $\mathrm{T}_{M}^{G} \mathrm{hd}(X)$ is semisimple. Therefore $J\left(\mathrm{~T}_{M}^{G} X\right) \subseteq$ $\mathrm{T}_{M}^{G} J(X)$. Using this inclusion multiple times proves the claim.

Lemma 4.30. The unique regular composition factor $D_{\text {reg }}(n)$ of $P_{S t}(G)$ lies in the head of $J^{r}\left(P_{S t}(G)\right)$.

Proof. The uniqueness of $D_{\text {reg }}(n)$ was proved in Corollary 3.3. We denote by $D_{\text {reg }}(L)$ the unique composition factor $D_{\text {reg }}(e)^{r} \times D_{\text {reg }}(1)^{s}$ of $P_{S t}(L)$. This composition factor lies in the head of $J^{r}\left(P_{S t}(L)\right)$ by Corollary 4.9. We know, that $J^{r}\left(P_{S t}(L)\right) \subseteq \mathrm{T}_{L}^{G} J^{r}\left(P_{S t}(G)\right)$. So $D_{\text {reg }}(L)$ is no composition factor of $\mathrm{T}_{L}^{G}\left(P_{S t}(G)\right) / \mathrm{T}_{L}^{G} J^{r}\left(P_{S t}(G)\right)$. Because $\mathrm{T}_{L}^{G} D_{\text {reg }}(n)=D_{\text {reg }}(L)$ by Proposition 3.8 this implies that $D_{\text {reg }}(n)$ is a composition factor of $J^{r}\left(P_{S t}(G)\right)$. So $D_{\text {reg }}(n)$ is a composition factor of hd $J^{i}\left(P_{S t}(G)\right)$ with $i \geq r$. 
On the other hand we know, that $D_{\text {reg }}(n)$ is a composition factor of the Steinberg module $S_{F}\left(1,1^{n}\right)$ and occurs with multiplicity one there. Furthermore, $P_{S t}(G)$ is a projective cover of $S_{F}\left(1,1^{n}\right)$. But by Corollary 2.24 of [DiGr] we know that $S_{F}\left(1,1^{n}\right)$ has exactly one composition factor in each modular HC-Series of the unipotent block. There are $r+1$ modular series in this block (see Prop. 2.10), so $S_{F}\left(1,1^{n}\right)$ has $r+1$ composition factors. So $D_{\text {reg }}(n)$ lies in the head of $J^{i}\left(P_{S t}(G)\right)$ with $i \leq r$.

The following observation is an easy consequence of the lemma and its proof above, but interesting in its own right.

Proposition 4.31. Let $e>1$ and let $r$ such that $n=e r+s$ with $s<e$ as above. Then the Loewy series of the $\ell$-modular reduction $S_{F}\left(1,1^{n}\right)$ of the ordinary Steinberg module as defined in section 2.3 is uniserial with $(r+1)$ composition factors. Furthermore hd $S_{F}\left(1,1^{n}\right) \cong D_{F}\left(1,1^{n}\right)$ and $\operatorname{soc} S_{F}\left(1,1^{n}\right) \cong D_{\text {reg }}(n)$.

Proof. From the construction of $D_{F}\left(1,1^{n}\right)$ we know that hd $S_{F}\left(1,1^{n}\right) \cong$ $D_{F}\left(1,1^{n}\right)$. As in the proof of Lemma 4.30 we have that $S_{F}\left(1,1^{n}\right)$ has exactly $(r+1)$ composition factors. Let $\pi: P_{S t}(G) \rightarrow S_{F}\left(1,1^{n}\right)$ be a minimal projective cover. We have $\pi\left(J^{i}\left(P_{S t}(G)\right)\right) \subseteq J^{i}\left(\pi\left(P_{S t}(G)\right)\right)=J^{i}\left(S_{F}\left(1,1^{n}\right)\right)$. Now the unique $e$-regular composition factor of $P_{S t}(G)$ is contained in hd $J^{r}\left(P_{S t}(G)\right)$ by Lemma 4.30. So its image cannot be zero in $S_{F}\left(1,1^{n}\right)$ and therefore $J^{r}\left(S_{F}\left(1,1^{n}\right)\right) \neq 0$. But $S_{F}\left(1,1^{n}\right)$ has only $(r+1)$ composition factors and so each radical layer contains exactly one composition factor and $J^{r}\left(S_{F}\left(1,1^{n}\right)\right) \cong \operatorname{soc} S_{F}\left(1,1^{n}\right) \cong D_{\text {reg }}(n)$.

The next corollary is an easy but important consequence of the Lemma 4.30.

Corollary 4.32. Let $\operatorname{Hom}_{F G}\left(J^{i}\left(P_{S t}(G)\right), \mathrm{R}_{L}^{G} D_{\text {reg }}(L)\right) \neq\{0\}$. Then this Hom space is 1-dimensional, we have $i=r$ and each homomorphism factors through $D_{\text {reg }}(n) \subseteq \mathrm{R}_{L}^{G} D_{\text {reg }}(L)$.

Proof. The Hom space has dimension at most 1, since by Frobenius reciprocity it is isomorphic to $\operatorname{Hom}_{F L}\left(\mathrm{~T}_{L}^{G} J^{i}\left(P_{S t}(G)\right), D_{\text {reg }}(L)\right)$ and $\mathrm{T}_{L}^{G} J^{i}\left(P_{S t}(G)\right) \leq$ $\mathrm{T}_{L}^{G} P_{S t}(G)=P_{S t}(L)$. Since $P_{S t}(L)$ has exactly one composition factor $D_{\text {reg }}(L)$ (see Cor. 4.9) the claim follows.

We already know that $D_{\text {reg }}(L)$ lies in the modular Harish-Chandra series $S_{F}\left(T, 1_{T}\right)$. Therefore the socle of $\mathrm{R}_{L}^{G} D_{\text {reg }}(L)$ consists only of irreducibles from this series (see Prop. 2.12), which are those $D_{F}(1, \lambda)$ with $\lambda e$-regular. Also $D_{\text {reg }}(n) \subseteq \mathrm{R}_{L}^{G} D_{\text {reg }}(L)$ is unique by Frobenius reciprocity. But $D_{\text {reg }}(n)$ is the only $e$-regular composition factor of $P_{S t}(G)$. So for any non-zero homomorphism $f \in$ $\operatorname{Hom}_{F G}\left(J^{i}\left(P_{S t}(G)\right), \mathrm{R}_{L}^{G} D_{\text {reg }}(L)\right)$ the socle of its image is $D_{\text {reg }}(G) \subseteq \mathrm{R}_{L}^{G} D_{\text {reg }}(L)$ and $i \leq r$. 
For $i=r$ we know that $D_{\text {reg }}(n)$ is a composition factor in the head of $J^{r}\left(P_{S t}(G)\right)$ by Lemma 4.30 and so everything is clear.

For $i<r$ we assume $\operatorname{dim} \operatorname{Hom}_{F L}\left(\mathrm{~T}_{L}^{G} J^{i}\left(P_{S t}(G)\right), D_{\text {reg }}(L)\right)=1$. Then $D_{\text {reg }}(L)$ is a composition factor of $P_{S t}(L) / J\left(\mathrm{~T}_{L}^{G} J^{i}\left(P_{S t}(G)\right)\right)$ which is a factor of $P_{S t}(L) / J^{i+1}\left(P_{S t}(L)\right)$. But this module has no composition factor $D_{\text {reg }}(L)$ by Lemma 4.30 which leads to a contradiction.

The next proposition shows, that if we prove the theorem for $L$ we immediately get the same theorem for any standard Levi subgroup $M$ with $L \leq M \leq G$.

Proposition 4.33. Let $L \lesseqgtr M \leq G$ be a standard Levi subgroup. Assume $J^{i}\left(P_{S t}(L)\right)=\mathrm{T}_{L}^{G} J^{i}\left(P_{S t}(G)\right)$ for some non-negative integer $i$. Then

$$
J^{i}\left(P_{S t}(M)\right)=\mathrm{T}_{M}^{G} J^{i}\left(P_{S t}(G)\right)
$$

Proof. We already know $J^{i}\left(P_{S t}(M)\right) \subseteq \mathrm{T}_{M}^{G} J^{i}\left(P_{S t}(G)\right)$ by Lemma 4.29. Now suppose this is a proper inclusion. This gives the following short exact sequence:

$$
0 \rightarrow J^{i}\left(P_{S t}(M)\right) \rightarrow \mathrm{T}_{M}^{G} J^{i}\left(P_{S t}(G)\right) \rightarrow X \rightarrow 0
$$

where $X \neq 0$. There are no cuspidal $F M$-modules in the unipotent block for any $L \lesseqgtr M$ since $r<\ell, s<e$. So we have $\mathrm{T}_{L}^{M} X \neq 0$. Using the exactness of $\mathrm{T}_{L}^{M}$ we get

$$
0 \rightarrow \mathrm{T}_{L}^{M} J^{i}\left(P_{S t}(M)\right) \rightarrow \mathrm{T}_{L}^{G} J^{i}\left(P_{S t}(G)\right) \rightarrow \mathrm{T}_{L}^{M} X \rightarrow 0
$$

This is a contradiction, since by assumption $\mathrm{T}_{L}^{G} J^{i}\left(P_{S t}(G)\right)=J^{i}\left(P_{S t}(L)\right) \subseteq$ $\mathrm{T}_{L}^{M} J^{i}\left(P_{S t}(M)\right)$

Now we are ready to proof the theorem.

of Theorem 4.28. In view of Proposition 4.33 it is enough to check the case $M=$ $L$. We use induction on $i$. The start $i=0$ is just identification $\mathrm{T}_{L}^{G} P_{S t}(G)=P_{S t}(L)$ (see Prop. 2.19). So we assume $J^{i}\left(P_{S t}(L)\right)=\mathrm{T}_{L}^{G} J^{i}\left(P_{S t}(G)\right)$. Since

$$
J^{i+1}\left(P_{S t}(L)\right)=J(F L) \cdot J^{i}\left(P_{S t}(L)\right) \leq J\left(\mathrm{~T}_{L}^{G} J^{i}\left(P_{S t}(G)\right) \leq \mathrm{T}_{L}^{G} J^{i+1}\left(P_{S t}(G)\right)\right.
$$

by Lemma 4.29 we know by inductive assumption hd $\mathrm{T}_{L}^{G} J^{i}\left(P_{S t}(G)\right)$ is contained in hd $J^{i}\left(P_{S t}(L)\right)$. By counting the factors we will prove that this is in fact an equality, so $J^{i+1}\left(P_{S t}(L)\right)=\mathrm{T}_{L}^{G} J^{i+1}\left(P_{S t}(G)\right)$

We first fix some notation. Let $X=D_{F}\left(1,1^{e}\right)^{\otimes \bar{r}} \otimes D_{\text {reg }}(e)^{\otimes t} \otimes D_{\text {reg }}(1)^{\otimes s}$ be an irreducible $F L$-module and let $M=G_{1} \times G_{2}=\mathrm{Gl}_{e \bar{r}}(q) \times \mathrm{Gl}_{e t+s}(q)$ be a Levi subgroup of $G$. We write $L_{1}=\mathrm{Gl}_{e}(q)^{\bar{r}}$ and $L_{2}=\mathrm{Gl}_{e}(q)^{t} \times \mathrm{Gl}_{1}(q)^{s}$. We also write $X=X^{\prime} \otimes D_{\text {reg }}\left(L_{2}\right)$ where $X^{\prime}=D_{F}\left(1,1^{r}\right)^{\otimes \bar{r}}$. 
The number of times that $X$ is a direct summand of $\mathrm{T}_{L}^{G}$ hd $J^{i}\left(P_{S t}(G)\right)$ will be denoted by $\omega(X)$. Since $\mathrm{T}_{L}^{G}$ hd $J^{i}\left(P_{S t}(G)\right)$ is semisimple we have

$$
\begin{aligned}
\omega(X) & =\operatorname{dim} \operatorname{Hom}_{F L}\left(\mathrm{~T}_{L}^{G} \operatorname{hd} J^{i}\left(P_{S t}(G)\right), X\right) \\
& =\operatorname{dim} \operatorname{Hom}_{F M}\left(\mathrm{~T}_{M}^{G} \text { hd } J^{i}\left(P_{S t}(G)\right), \mathrm{R}_{L}^{M} X\right) \\
& =\operatorname{dim} \operatorname{Hom}_{F M}\left(\mathrm{~T}_{M}^{G} \operatorname{hd} J^{i}\left(P_{S t}(G)\right), \mathrm{R}_{L_{1}}^{G_{1}} X^{\prime} \otimes \mathrm{R}_{L_{2}}^{G_{2}} D_{r e g}\left(L_{2}\right)\right)
\end{aligned}
$$

Since $\bar{r}<\ell$ and $X^{\prime}$ is cuspidal, by [Di1] $\mathrm{R}_{L_{1}}^{G_{1}} X^{\prime}$ is semisimple and its irreducible constituents are determined by the semisimple $F$-algebra $\operatorname{End}_{F G_{1}}\left(\mathrm{R}_{L_{1}}^{G_{1}} X^{\prime}\right)=$ $\mathcal{H}_{f, q^{e}}\left(S_{\bar{r}}\right) \cong F S_{\bar{r}}$. Let $Y$ be any irreducible $F G_{1}$-module in the modular HarishChandra series $S\left(L_{1}, X^{\prime}\right)$. Denote by $m(Y)$ multiplicity of $Y$ in the semisimple $F G_{1}$-module $\mathrm{R}_{L_{1}}^{G_{1}} X^{\prime}$. The multiplicity $m(Y)$ is the same as the multiplicity of $X^{\prime}$ in $\mathrm{T}_{L_{1}}^{G_{1}} Y$ by Frobenius reciprocity.

As in the proof of Corollary 4.32 soc $\mathrm{R}_{L_{2}}^{G_{2}} D_{\text {reg }}\left(L_{2}\right)$ consists only of $e$-regular composition factors and $D_{\text {reg }}\left(G_{2}\right)$ has multiplicity 1 in there. Since the simple submodules of $\mathrm{T}_{M}^{G}$ hd $J^{i}\left(P_{S t}(G)\right)$ are of the form $A \otimes B$ with $A$ a composition factor of $P_{S t}\left(G_{1}\right)$ and $B$ a composition factor of $P_{S t}\left(G_{2}\right)$ we can substitute $D_{\text {reg }}\left(G_{2}\right)$ for $\mathrm{R}_{L_{2}}^{G_{2}} D_{\text {reg }}\left(L_{2}\right)$. So

$$
\begin{aligned}
\omega(X) & =\sum_{Y \in S\left(L_{1}, X^{\prime}\right)} m(Y) \cdot \operatorname{dim} \operatorname{Hom}_{F M}\left(\mathrm{~T}_{M}^{G} \operatorname{hd} J^{i}\left(P_{S t}(G)\right), Y \otimes D_{\text {reg }}\left(G_{2}\right)\right) \\
& =\sum_{Y \in S\left(L_{1}, X^{\prime}\right)} m(Y) \cdot \operatorname{dim} \operatorname{Hom}_{F G}\left(\mathrm{hd} J^{i}\left(P_{S t}(G)\right), \mathrm{R}_{M}^{G} Y \otimes D_{r e g}\left(G_{2}\right)\right)
\end{aligned}
$$

Now hd $J^{i}\left(P_{S t}(G)\right)$ is semisimple and $\mathrm{R}_{M}^{G} Y \otimes D_{\text {reg }}\left(G_{2}\right)$ is simple (by [DiJa1], Thm 5.2). So we can lift each homomorphism hd $J^{i}\left(P_{S t}(G)\right) \rightarrow \mathrm{R}_{M}^{G} Y \otimes D_{\text {reg }}\left(G_{2}\right)$ to a homomorphism $J^{i}\left(P_{S t}(G)\right) \rightarrow \mathrm{R}_{M}^{G} Y \otimes D_{\text {reg }}\left(G_{2}\right)$. We get

$$
\begin{aligned}
\omega(X) & =\sum_{Y \in S\left(L_{1}, X^{\prime}\right)} m(Y) \operatorname{dim} \operatorname{Hom}_{F G}\left(J^{i}\left(P_{S t}(G)\right), \mathrm{R}_{M}^{G} Y \otimes D_{r e g}\left(G_{2}\right)\right) \\
& =\sum_{Y \in S\left(L_{1}, X^{\prime}\right)} m(Y) \operatorname{dim} \operatorname{Hom}_{F M}\left(\mathrm{~T}_{M}^{G} J^{i}\left(P_{S t}(G)\right), Y \otimes D_{r e g}\left(G_{2}\right)\right) \\
& =\operatorname{dim} \operatorname{Hom}_{F M}\left(\mathrm{~T}_{M}^{G} J^{i}\left(P_{S t}(G)\right), \mathrm{R}_{L_{1}}^{G_{1}} X^{\prime} \otimes D_{r e g}\left(G_{2}\right)\right)
\end{aligned}
$$

By Proposition 4.33 and the inductive assumption we know that $\mathrm{T}_{M}^{G} J^{i}\left(P_{S t}(G)\right)=$ $J^{i}\left(P_{S t}(M)\right)$. Furthermore we can describe the radical series of $P_{S t}(M)$ in terms of the radical series of $P_{S t}\left(G_{1}\right)$ and $P_{S t}\left(G_{2}\right)$ as in Proposition 4.8:

$$
J^{i}\left(P_{S t}(M)\right)=\sum_{k=0}^{i} J^{i-k}\left(P_{S t}\left(G_{1}\right)\right) \otimes J^{k}\left(P_{S t}\left(G_{2}\right)\right)
$$


Let $0 \neq f \in \operatorname{Hom}_{F M}\left(J^{i}\left(P_{S t}(M)\right), \mathrm{R}_{L_{1}}^{G_{1}} X^{\prime} \otimes D_{\text {reg }}\left(G_{2}\right)\right)$. Let $\tilde{f}$ be the restriction of $f$ to the submodule $J^{i-k}\left(P_{S t}\left(G_{1}\right)\right) \otimes J^{k}\left(P_{S t}\left(G_{2}\right)\right)$. By general theory $\tilde{f}=g \otimes h$ with $h: J^{k}\left(P_{S t}\left(G_{2}\right)\right) \rightarrow D_{\text {reg }}\left(G_{2}\right)$ being an $F G_{2}$-module homomorphism. By Corollary 4.32 every homomorphism $h^{\prime}: J^{k}\left(P_{S t}\left(G_{2}\right)\right) \rightarrow \mathrm{R}_{L_{2}}^{G_{2}} D_{\text {reg }}\left(L_{2}\right)$ factors through $D_{\text {reg }}\left(G_{2}\right)$ and is almost always zero. So we can substitute $D_{\text {reg }}\left(G_{2}\right)$ by $\mathrm{R}_{L_{2}}^{G_{2}} D_{\text {reg }}\left(L_{2}\right)$ in the equation above:

$$
\begin{aligned}
\omega(X) & =\operatorname{dim} \operatorname{Hom}_{F M}\left(\mathrm{~T}_{M}^{G} J^{i}\left(P_{S t}(G)\right), \mathrm{R}_{L_{1}}^{G_{1}} X^{\prime} \otimes \mathrm{R}_{L_{2}}^{G_{2}} D_{r e g}\left(L_{2}\right)\right) \\
& =\operatorname{dim} \operatorname{Hom}_{F L}\left(\mathrm{~T}_{L}^{G} J^{i}\left(P_{S t}(G)\right), X^{\prime} \otimes D_{\text {reg }}\left(L_{2}\right)\right) \\
& =\operatorname{dim} \operatorname{Hom}_{F L}\left(J^{i}\left(P_{S t}(L)\right), X^{\prime} \otimes D_{\text {reg }}\left(L_{2}\right)\right)
\end{aligned}
$$

The last line says that the number of times, that $X^{\prime} \otimes D_{\text {reg }}\left(L_{2}\right)$ is a composition factor of hd $J^{i}\left(P_{S t}(L)\right)$ is the same as for $\mathrm{T}_{L}^{G}$ hd $J^{i}\left(P_{S t}(G)\right)$.

We get each composition factor $Y$ of $P_{S t}(L)$ by permuting the factors of $X$ and by changing $\bar{r}$ (see Cor 3.15). But for any permutation $Y$ of the factors of $X$ we have again the same numbers on both sides, by Frobenius reciprocity and the fact, that $\mathrm{R}_{L}^{G} X \cong \mathrm{R}_{L}^{G} Y$. But the arguments also do not depend on the value of $\bar{r}$. So we have that $\mathrm{T}_{L}^{G}$ hd $J^{i}\left(P_{S t}(G)\right)$ and hd $J^{i}\left(P_{S t}(L)\right)$ have the same composition factors. This proves the theorem.

\subsection{Calculation of the Loewy Series}

In this subsection we again assume $e>1$. The Theorem 4.39 below is still valid in this case (just compare Prop. 4.27) but the proofs need this additional assumption.

There remains one problem to solve in calculating the Loewy series. It is illustrated by the following example:

Let $G=\mathrm{Gl}_{4}(q)$ with $q=9$ and $\ell=5$ so $e=2$. The structure of the radical series of $P_{S t}\left(\mathrm{Gl}_{2}(q)\right)$ given by Theorem 4.4 .

$$
P_{S t}\left(\mathrm{Gl}_{2}(q)\right)=\begin{gathered}
{\left[1^{2}\right]} \\
{\left[1^{2}\right] \oplus[2]} \\
{\left[1^{2}\right]}
\end{gathered}
$$

where we write just $[\lambda]$ for the modular irreducible module $D_{F}(1, \lambda)$. Writing $[\lambda][\mu]$ for the irreducible $F L$-module $D_{F}(1, \lambda) \otimes D_{F}(1, \mu)$ we obtain for the radical series 
of $P_{S t}(L)$ :

$$
\begin{gathered}
{\left[1^{2}\right]\left[1^{2}\right]} \\
{\left[1^{2}\right]\left[1^{2}\right] \oplus\left[1^{2}\right]\left[1^{2}\right] \oplus\left[1^{2}\right][2] \oplus[2]\left[1^{2}\right]} \\
P_{S t}(L)=\left[1^{2}\right]\left[1^{2}\right] \oplus\left[1^{2}\right]\left[1^{2}\right] \oplus\left[1^{2}\right]\left[1^{2}\right] \oplus\left[1^{2}\right][2] \oplus[2]\left[1^{2}\right] \oplus[2][2] \\
{\left[1^{2}\right]\left[1^{2}\right] \oplus\left[1^{2}\right]\left[1^{2}\right] \oplus\left[1^{2}\right][2] \oplus[2]\left[1^{2}\right]} \\
{\left[1^{2}\right]\left[1^{2}\right]}
\end{gathered}
$$

From Theorem 3.5 we know that $[4],\left[2^{2}\right],\left[21^{2}\right]$ and $\left[1^{4}\right]$ could be composition factors of $P_{S t}(G)$. The following tabular gives their restriction to $L$ :

\begin{tabular}{l|l}
$M$ & $\mathrm{~T}_{L}^{G} M$ \\
\hline$[4]$ & {$[2][2]$} \\
{$\left[2^{2}\right]$} & {$\left[1^{2}\right]\left[1^{2}\right]$} \\
{$\left[21^{2}\right]$} & {$\left[1^{2}\right][2] \oplus[2]\left[1^{2}\right]$} \\
{$\left[1^{4}\right]$} & {$\left[1^{2}\right]\left[1^{2}\right]$}
\end{tabular}

So [4] and $\left[21^{2}\right]$ can be uniquely identified by their respective restriction, but $\left[2^{2}\right]$ and $\left[1^{4}\right]$ can not be distinguished this way. So at this point we can say that the radical structure of $P_{S t}(G)$ looks something like this:

$$
\begin{gathered}
{[*]} \\
{[*] \oplus[*] \oplus\left[21^{2}\right]} \\
{[*] \oplus[*] \oplus[*] \oplus\left[21^{2}\right] \oplus[4]} \\
{[*] \oplus[*] \oplus\left[21^{2}\right]} \\
{[*]}
\end{gathered}
$$

where $[*]$ stands for either $\left[1^{4}\right]$ or $\left[2^{2}\right]$. We see that this approach leaves the unsolved problem to identify the correct source if two irreducible modules have the same restriction or if the restriction of two irreducible modules have composition factors in common, which can happen in bigger examples.

The identification is solved in the following propositions:

Proposition 4.34. Let $D_{F}(1, \mu)$ be a composition factor of $P_{S t}(G)$ and assume that $\mu^{\prime}=\alpha^{\prime}+e \rho^{\prime}$ where $\alpha$ is e-regular and a partition of $r \geq e$. Then $D_{F}(1, \mu)$ is uniquely determined by $\mathrm{T}_{L}^{G} D_{F}(1, \mu)$ with $L=\mathrm{Gl}_{e}(q) \times \mathrm{Gl}_{n-e}(q)$.

Proof. We claim, that $\mathrm{T}_{L}^{G} D_{F}(1, \mu)$ contains a direct summand $D_{\text {reg }}(e) \otimes D_{F}(1, \lambda)$ and that $\lambda \vdash n-e$ uniquely determines $\mu$. Let $\beta \vdash|\alpha|-e$ be the partition corresponding to $D_{\text {reg }}(|\alpha|-e)$. Define $\lambda \vdash n-e$ be $\lambda^{\prime}=\beta^{\prime}+e \rho^{\prime}$. The proof of 
our claim is just a careful analysis of the proof of theorem 3.7. $D_{\text {reg }}(e) \otimes D_{F}(1, \lambda)$ is a direct summand, we just look at $x=1$ in the Mackey decomposition.

Now suppose $D_{\text {reg }}(e) \otimes D_{F}(1, \lambda)$ is a composition factor of $\mathrm{T}_{L}^{G} D(1, \mu)$. Each composition factor has the form

$$
\left(\mathrm{R}_{\mathrm{Gl}_{a}(q) \times \mathrm{Gl}_{e-a}(q)}^{\mathrm{Gl}_{2}(q)} D_{r e g}(a) \otimes D_{F}\left(1,\left(e \eta^{\prime}\right)^{\prime}\right)\right) \otimes\left(\mathrm{R}_{\mathrm{Gl}_{c}(q) \times \mathrm{Gl}_{n-e-c}(q)}^{\mathrm{Gl}_{n-e}(q)} D_{r e g}(c) \otimes D_{F}\left(1,\left(e \gamma^{\prime}\right)^{\prime}\right)\right)
$$

If the first factor is $D_{\text {reg }}(e)$ we have $a=e$ and this implies $D_{F}\left(1,\left(e \gamma^{\prime}\right)^{\prime}\right) \cong$ $D_{F}\left(1,\left(e \rho^{\prime}\right)^{\prime}\right)$ by construction of $\lambda$. But by construction of $\gamma$ (in the proof of 3.7) we know $\gamma=\rho$ and therefore $\lambda$ determines $\mu$.

The remaining case, where the $e$-regular part of the partition is smaller than $e$, needs more machinery. We start by a more general observation, which will be used subsequently:

Proposition 4.35. Let $P$ be a projective module $F G$-module and let $\mathcal{E}=$ $\operatorname{End}_{F G}(P)$ be the endomorphism ring. Then there is a bijection as partially ordered sets between the right ideals of $\mathcal{E}$ (henceforth $\mathcal{E}$-ideals) and those right submodules $U \subseteq P$ such that $P$ is also a projective generator of $U$ i.e. there exists an integer $n$ and a surjective homomorphism $P^{n} \rightarrow U$ (henceforth called $P$-mod). The bijection is given by

$$
\begin{gathered}
\mathcal{F}: \mathcal{E} \text {-ideals } \rightarrow P \text {-mod } \quad I \mapsto I \cdot P:=\sum_{f \in I} \operatorname{Im}(f) \\
\mathcal{G}: P \text {-mod } \rightarrow \mathcal{E} \text {-ideals } \quad U \mapsto \operatorname{Hom}_{k G}(P, U)
\end{gathered}
$$

Proof. This is a specialisation of a far more general theory about Schur functors which can be found for example in [BrDiKl] chapter 3. From there we know that our functors are mutually inverse equivalences of categories between mod-E $\mathcal{E}$ and the full sub-category of mod- $k G$ such that $P$ is a projective generator (Theorem $3.1 \mathrm{~d}$ and Lemma $3.1 \mathrm{f}$ in $[\mathrm{BrDiKl}]$ ). So we just need to show that in case of our sub-categories $\mathcal{F} \circ \mathcal{G}$ and $\mathcal{G} \circ \mathcal{F}$ are in fact identities.

First we have to show that the images of our functors are of the form we claimed. For any submodule $U \subseteq P$ we have that $\operatorname{Hom}_{k G}(P, U)$ is a right ideal in $\mathcal{E}$. On the other hand for any right ideal $I \leq \mathcal{E}$ we have that $I \cdot P=\sum_{f \in I} \operatorname{Im}(f) \subseteq P$ and since $I$ is finitely generated, $P$ is a projective generator of this submodule.

For any $U \in P$-mod we have $\operatorname{Hom}_{k G}(P, U) \cdot U \subseteq U$ by definition. But since $P$ is a projective generator for $U$, we also have $U \subseteq \sum_{f \in \operatorname{Hom}_{k G}(P, U)} \operatorname{Im}(f)$. So $\mathcal{F} \circ \mathcal{G}=\operatorname{id}_{P \text {-mod }}$. For any $I \in \mathcal{E}$-ideals we have $I \subseteq \operatorname{Hom}_{k G}(P, I \cdot P)$ from the definition and $\operatorname{Hom}_{k G}(P, I \cdot P) \cong I$ from the equivalence of categories. Together this gives $\mathcal{G} \circ \mathcal{F}=\operatorname{id}_{P \text {-mod }}$.

The partial orderings of this sets are clearly respected by the functors, since $I \subseteq J$ implies $I \cdot P \subseteq J \cdot P$ and $U \subseteq V$ implies $\operatorname{Hom}_{k G}(P, U) \subseteq \operatorname{Hom}_{k G}(P, V)$. 
We want to apply this proposition to the case where $P=\mathrm{R}_{L}^{G} P_{S t}(G)$ and calculate some part of the radical series of $P$ from the radical series of $\mathcal{E}=$ $\operatorname{End}_{F G}\left(\mathrm{R}_{L}^{G} P_{S t}(G)\right)$. From Proposition 4.21 we know, that all the composition factors which we still want to identify occur in the head of $P$. Unfortunately, we do not know $\mathcal{E}$ well enough. We only know the sub-algebra $H$ well enough. But as the following propositions will show, this is good enough for our purpose.

First we need to know how the labelling of the PIMs and therefore the irreducibles of $\mathcal{E}$ and $P$ correspond in our case:

Proposition 4.36. Let $f$ be a primitive idempotent of $\mathcal{E}$. Then hd $f \mathcal{E}$ is an irreducible $H / J(H)$ module and therefore labelled by a pair of partitions $\left(\lambda_{1}, \lambda_{2}\right)$ with $\lambda_{1} \vdash r$ and $\lambda_{2} \vdash s$. Then $f \mathcal{E} \cdot P$ is the PIM of $G$ corresponding to the label $\mu$ with $\mu^{\prime}=\lambda_{2}^{\prime}+\left(e \lambda_{1}^{\prime}\right)^{\prime}$.

Proof. The first claim is a restatement of Proposition 4.22. The second claim follows if we just look at the heads of the PIMs involved. Under the map $\mathcal{F}$ the head of $\mathcal{E}$ just maps to the head of $P$. But the head of $\mathcal{E}$ is just the semisimple algebra $F S_{r} \otimes F S_{s}$ and the head of $P$ is just $\mathrm{R}_{L}^{G} C$ with $C$ being the unique cuspidal $F L$-module in the unipotent block. The correspondence between those has been described in the work of Dipper (see [Di3], §5).

We write $\pi$ for the map that is projection from $\mathrm{R}_{L}^{G} P_{S t}(L)$ onto $P_{S t}(G)$ followed by the inclusion from $P_{S t}(G)$ into $\mathrm{R}_{L}^{G} P_{S t}(G)$. Form the definition of $H$ it is clear that all endomorphisms $f: \mathrm{R}_{L}^{G} P_{S t}(L) \rightarrow P_{S t}(G) \hookrightarrow \mathrm{R}_{L}^{G} P_{S t}(L)$ are contained in $H$. So in fact $\pi \mathcal{E}=\pi H$. So we can look at the radical series of $\pi H$ instead of the radical series of $\pi \mathcal{E}$ to determine the unknown in the radical series of $P_{S t}(G)$. Because all $\mathcal{E}$-ideals of $\pi \mathcal{E}$ are $H$-ideals it is clear that $\mathcal{F}$ gives a surjective map from $H$-ideals of $\pi H$ to the $P$-mod submodules of $P_{S t}(G)$. But it is not clear, that this map also respects the radical series and is injective. This is the claim of the next proposition:

Proposition 4.37. Let $\lambda$ be a partition of $r, M=\mathrm{Gl}_{e r}(q) \times \mathrm{Gl}_{s}(q)$ and let $k$ be a non-negative integer. Furthermore suppose that the number of composition factors of type $\left[1^{e}\right]$ in $P_{S t}\left(\mathrm{Gl}_{e}(q)\right)$ is at least 3. Then the number of times, that $S^{\lambda} \otimes S^{\left(1^{s}\right)}$ is a composition factor of the $k^{\text {th }}$ radical layer of $\pi H$ is the same as the number of times, that $\mathrm{R}_{M}^{G} D_{F}\left(1,\left(e \lambda^{\prime}\right)^{\prime}\right) \otimes D_{\text {reg }}(s)$ is a composition factor of the $k^{\text {th }}$ radical layer of $P_{S t}(G)$.

Proof. We already know by Proposition 4.36 and Proposition 3.5 that the composition factors $\mathrm{R}_{M}^{G} D_{F}\left(1,\left(e \lambda^{\prime}\right)^{\prime}\right) \otimes D_{F}\left(1,1^{s}\right)$ (and note that $D_{\text {reg }}(s)=D_{F}\left(1,1^{s}\right)$ ) are the only ones which occur in $P_{S t}(G)$ and can be "seen" by $\mathcal{E}$ and $H$. We also know from Proposition 4.23 that any simple $\mathcal{E}$-module is a simple $H$-module and therefore any semisimple $\mathcal{E}$-module is also semisimple as $H$-module. This means 
that the radical series of $\pi \mathcal{E}$ viewed as $H$-modules already has the characteristic that the various factors are semisimple. So we get inductively that the $k^{\text {th }}$ radical layer of $\pi \mathcal{E}$ is included in the $k^{t h}$ radical layer of $\pi H$, provided the $(k-1)^{t h}$ layers are equal. So it is enough to show that the layers have the same number of composition factors.

We start by counting the number of those composition factors of the $k^{\text {th }}$ radical layer of $P_{S t}(G)$ which are of the form $\mathrm{R}_{M}^{G} D_{F}\left(1,\left(e \lambda^{\prime}\right)^{\prime}\right) \otimes D_{\text {reg }}(s)$. Since $\mathrm{T}_{L}^{G} \mathrm{R}_{M}^{G} D_{F}\left(1,\left(e \lambda^{\prime}\right)^{\prime}\right) \otimes D_{\text {reg }}(s) \cong m(\lambda) D_{F}\left(1,1^{e}\right)^{\otimes r} \otimes D_{\text {reg }}(1)^{\otimes s}$ by Corollary 3.15 we count the number of composition factors of type $D_{F}\left(1,1^{e}\right)^{\otimes r} \otimes D_{\text {reg }}(1)^{\otimes s}$ in the $k^{\text {th }}$ layer of $P_{S t}(L)$ instead. As in Corollary $3.15 m(\lambda)$ denotes the multiplicity of $D_{F}\left(1,1^{e}\right)^{\otimes r}$ in $\mathrm{T}_{\mathrm{Gl}_{e}(q)^{r}}^{\mathrm{Gl}_{e}(q)} D_{F}\left(1,\left(e \lambda^{\prime}\right)^{\prime}\right)$.

From the structure of $P_{S t}\left(\mathrm{Gl}_{e}(q)\right)$ and the construction of the radical series we get that this number is (and here we use that $\left[1^{e}\right]$ occurs in each layer of $P_{S t}\left(\mathrm{Gl}_{e}(q)\right)$ )

$$
\sum_{\rho \vdash r} \operatorname{comp}(k, d, \rho) \operatorname{dim}_{F} M^{\rho} .
$$

Now we want to count the factors corresponding to $\mathrm{R}_{M}^{G} D_{F}\left(1,\left(e \lambda^{\prime}\right)^{\prime}\right) \otimes D_{\text {reg }}(s)$ in the $k^{\text {th }}$ radical layer of $\pi H$ and show that the number is equal. From Theorem 4.24 we get for the number of composition factors of type $S^{\lambda} \otimes S^{\left(1^{s}\right)}$ in the $k^{t h}$ radical layer of the PIM of $H$ labelled by $\left(1^{r}, 1^{s}\right)$ :

$$
\sum_{\rho \vdash r} \operatorname{comp}(k, d, \rho) \cdot \operatorname{dim}_{F} \operatorname{Hom}_{F S_{r} \otimes F S_{s}}\left(S^{\lambda} \otimes S^{\left(1^{s}\right)},\left(S^{\left(1^{r}\right)} \otimes S^{\left(1^{s}\right)}\right) \otimes M^{(\rho,(s))}\right)
$$

For counting purposes we can just ignore the second part of all these modules. To compare this expression with the previous one we have to sum over all possible partitions $\lambda \vdash r$ and we have to take into account the multiplicity we get when applying HC-restriction. This gives:

$$
\sum_{\lambda \vdash r} m(\lambda) \sum_{\rho \vdash r} \operatorname{comp}(k, d, \rho) \cdot \operatorname{dim}_{F} \operatorname{Hom}_{F S_{r}}\left(S^{\lambda}, S^{\left(1^{r}\right)} \otimes M^{\rho}\right)
$$

Since tensoring with $S^{\left(1^{r}\right)}$ induces a bijection on the irreducible $F S_{r}$-modules we can ignore it, since we sum over all $\lambda \vdash r$. To get our desired result, we only have to prove the following equation for any $\rho \vdash r$ :

$$
\sum_{\lambda \vdash r} m(\lambda) \operatorname{dim}_{F} \operatorname{Hom}_{F S_{r}}\left(S^{\lambda}, M^{\rho}\right) \stackrel{!}{=} \operatorname{dim}_{F} M^{\rho}
$$

But we have $\operatorname{dim}_{F} M^{\rho}=\sum_{\lambda \vdash r} \operatorname{dim}_{F} \operatorname{Hom}_{F S_{r}}\left(S^{\lambda}, M^{\rho}\right) \cdot \operatorname{dim}_{F} S^{\lambda}$ since $F S_{r}$ is semisimple and

$$
\begin{aligned}
\operatorname{dim} S^{\lambda} & =\text { number of standard } \lambda \text {-tableaux } \\
& =\operatorname{dim}_{F} \operatorname{Hom}_{F S_{r}}\left(S^{\lambda},\left(S^{(1)}\right)^{\otimes r} \uparrow^{F S_{r}}\right)
\end{aligned}
$$


(see [Ja1] $§ 8, \S 16$ ). But the last number is equal to $m(\lambda)$ since $F S_{r}$ is isomorphic to the endomorphism ring $\operatorname{End}_{F L}\left(\mathrm{R}_{L}^{G} D_{F}\left(1,1^{e}\right)^{\otimes r}\right)$ (see [Di1]).

The missing case, where $P_{S t}\left(\mathrm{Gl}_{e}(q)\right)$ has the form

$$
\begin{gathered}
D_{F}\left(1,1^{e}\right) \\
D_{r e g}(e) \\
D_{F}\left(1,1^{e}\right)
\end{gathered}
$$

can be treated similarly. The only difference is that every other layer of $P_{S t}(L)$ has no composition factor of the form $D_{F}\left(1,1^{e}\right)^{\otimes r} \otimes D_{F}(1,1)^{\otimes s}$. So the formulae must be adjusted. We have:

Corollary 4.38. Let $\lambda$ be a partition of $r, M=\mathrm{Gl}_{e r}(q) \times \mathrm{Gl}_{s}(q)$ and let $k$ be a non-negative integer. Furthermore, suppose that the number of composition factors of type $\left[1^{e}\right]$ in $P_{S t}\left(\mathrm{Gl}_{e}(q)\right)$ is 2. Then the number of times that $S^{\lambda} \otimes S^{\left(1^{s}\right)}$ is a composition factor of the $k^{\text {th }}$ radical layer of $\pi H$ is the same as the number of times that $\mathrm{R}_{M}^{G} D_{F}\left(1,\left(e \lambda^{\prime}\right)^{\prime}\right) \otimes D_{\text {reg }}(s)$ is a composition factor of the $2 k^{\text {th }}$ radical layer of $P_{S t}(G)$.

We close this section with two examples. First we finish the example above:

The $H$-structure of the corresponding PIM of $P_{S t}(G)$ looks like this with $[\lambda]$ written for $S^{\lambda}$ :

$$
\begin{gathered}
{\left[1^{2}\right]} \\
{\left[1^{2}\right] \oplus[2]} \\
{\left[1^{2}\right] \oplus\left[1^{2}\right] \oplus[2]} \\
{\left[1^{2}\right] \oplus[2]} \\
{\left[1^{2}\right]}
\end{gathered}
$$

Now $\left[1^{2}\right]$ corresponds to $\left[1^{4}\right]=D_{F}\left(1,1^{4}\right)$ and [2] corresponds to $\left[2^{2}\right]=D_{F}\left(1,2^{2}\right)$. By the Proposition 4.37 and the previous calculations this means, that the radical structure of the Steinberg PIM of $\mathrm{Gl}_{4}(q)$ is

$$
\begin{gathered}
{\left[1^{4}\right]} \\
{\left[1^{4}\right] \oplus\left[2^{2}\right] \oplus\left[21^{2}\right]} \\
P_{S t}\left(\mathrm{Gl}_{4}(q)\right)=\left[1^{4}\right] \oplus\left[1^{4}\right] \oplus\left[2^{2}\right] \oplus\left[21^{2}\right] \oplus[4] \\
{\left[1^{4}\right] \oplus\left[2^{2}\right] \oplus\left[21^{2}\right]} \\
{\left[1^{4}\right]}
\end{gathered}
$$

The second example is a little bigger, to also illustrate the other identification process.

We use $G=\operatorname{Gl}_{6}(q)$ with $q=13$ and $\ell=7$, so again $e=2$. The structure of the radical series of $P_{S t}\left(\mathrm{Gl}_{2}(q)\right)$ is

$$
P_{S t}\left(\mathrm{Gl}_{2}(q)\right)=\begin{gathered}
{\left[1^{2}\right]} \\
{\left[1^{2}\right] \oplus[2]} \\
{\left[1^{2}\right]} \\
{\left[1^{2}\right]}
\end{gathered}
$$


To use Proposition 4.34 we also need to know the radical series of $P_{S t}\left(\mathrm{Gl}_{4}(q)\right)$, which can be calculated analogously to the previous example. We get:

$$
\begin{gathered}
{\left[1^{4}\right]} \\
{\left[1^{4}\right] \oplus\left[2^{2}\right] \oplus\left[21^{2}\right]} \\
{\left[1^{4}\right] \oplus\left[1^{4}\right] \oplus\left[2^{2}\right] \oplus\left[21^{2}\right] \oplus[4]} \\
P_{S t}\left(\mathrm{Gl}_{4}(q)\right)=\left[1^{4}\right] \oplus\left[1^{4}\right] \oplus\left[2^{2}\right] \oplus\left[2^{2}\right] \oplus\left[21^{2}\right] \\
{\left[1^{4}\right] \oplus\left[1^{4}\right] \oplus\left[2^{2}\right]} \\
{\left[1^{4}\right] \oplus\left[2^{2}\right]} \\
{\left[1^{4}\right]}
\end{gathered}
$$

Now we could calculate explicitly the radical series of $P_{S t}\left(\mathrm{Gl}_{4}(q)\right) \otimes P_{S t}\left(\mathrm{Gl}_{2}(q)\right)$. But this is not necessary for our purpose. By Proposition 4.34 we only need to know where the elements $[\lambda][2]$ are and since [2] occurs only once in the radical series of $P_{S t}\left(\mathrm{Gl}_{2}(q)\right)$ (in the first layer) this is easy. So we start by calculating the Harish-Chandra restriction of the composition factors that might occur, where $L=\mathrm{Gl}_{4}(q) \times \mathrm{Gl}_{2}(q):$

\begin{tabular}{l|l}
$M$ & $\mathrm{~T}_{L}^{G} M$ \\
\hline$[6]$ & {$[4][2]$} \\
{$\left[41^{2}\right]$} & {$[4]\left[1^{2}\right] \oplus\left[21^{2}\right][2]$} \\
{$\left[2^{3}\right]$} & {$\left[21^{2}\right]\left[1^{2}\right] \oplus\left[2^{2}\right][2]$} \\
{$\left[21^{4}\right]$} & {$\left[21^{2}\right]\left[1^{2}\right] \oplus\left[1^{4}\right][2]$} \\
{$\left[3^{2}\right]$} & {$\left[2^{2}\right]\left[1^{2}\right]$} \\
{$\left[2^{2} 1^{2}\right]$} & {$\left[2^{2}\right]\left[1^{2}\right] \oplus\left[1^{4}\right]\left[1^{2}\right]$} \\
{$\left[1^{6}\right]$} & {$\left[1^{4}\right]\left[1^{2}\right]$}
\end{tabular}

For the elements $\left[3^{2}\right],\left[2^{2} 1^{2}\right]$ and $\left[1^{6}\right]$ we need to know the combinatorial data from Theorem 4.24:

\begin{tabular}{c|c|c|c}
$k$ & $\operatorname{comp}(k, 4,(3))$ & $\operatorname{comp}(k, 4,(21))$ & $\operatorname{comp}\left(k, 4,\left(1^{3}\right)\right)$ \\
\hline 0 & $\left(0^{3}\right)$ & - & - \\
\hline 1 & - & $\left(0^{2} 1\right)$ & - \\
\hline 2 & - & $\left(0^{2} 2\right),\left(1^{2} 0\right)$ & - \\
\hline 3 & $\left(1^{3}\right)$ & $\left(0^{2} 3\right)$ & $(012)$ \\
\hline 4 & - & $\left(1^{2} 2\right),\left(2^{2} 0\right)$ & $(013)$ \\
\hline 5 & - & $\left(1^{2} 3\right),\left(2^{2} 1\right)$ & $(023)$ \\
\hline 6 & $\left(2^{3}\right)$ & $\left(3^{2} 0\right)$ & $(123)$ \\
\hline 7 & - & $\left(3^{2} 1\right),\left(2^{2} 3\right)$ & - \\
\hline 8 & - & $\left(3^{2} 2\right)$ & - \\
\hline 9 & $\left(3^{3}\right)$ & - & -
\end{tabular}


The next table gives the dimensions $\operatorname{dim}_{F} \operatorname{Hom}_{F S_{3}}\left(S^{\lambda}, M^{\rho} \otimes S^{\left(1^{3}\right)}\right)$

\begin{tabular}{l|ccc} 
& $M^{(3)}$ & $M^{(21)}$ & $M^{\left(1^{3}\right)}$ \\
\hline$S^{(3)}$ & 0 & 0 & 1 \\
$S^{(21)}$ & 0 & 1 & 2 \\
$S^{\left(1^{3}\right)}$ & 1 & 1 & 1
\end{tabular}

For better readability we give the result in a table. The numbers in the column labelled by $[\lambda]$ give the number of composition factors of type $[\lambda]$ in the respective layers. The radical series of $P_{S t}\left(\mathrm{Gl}_{6}(q)\right)$ is:

\begin{tabular}{|c|ccccccc|}
\hline layer & {$[6]$} & {$\left[41^{2}\right]$} & {$\left[2^{3}\right]$} & {$\left[21^{4}\right]$} & {$\left[3^{2}\right]$} & {$\left[2^{2} 1^{2}\right]$} & {$\left[1^{6}\right]$} \\
\hline 0 & & & & & & & 1 \\
1 & & & & 1 & & 1 & 1 \\
2 & & 1 & 1 & 1 & & 2 & 2 \\
3 & 1 & 1 & 1 & 2 & 1 & 3 & 3 \\
4 & & 1 & 2 & 2 & 1 & 4 & 3 \\
5 & & & 1 & 2 & 1 & 4 & 3 \\
6 & & & 1 & 1 & 1 & 3 & 3 \\
7 & & & & 1 & & 2 & 2 \\
8 & & & & & & 1 & 1 \\
9 & & & & & & & 1 \\
\hline
\end{tabular}

We can shape our result into explicit formulas, only containing the combinatorial data and the dimensions of Hom-spaces of the symmetric group. We just combine Propositions 4.34 and 4.37 .

Theorem 4.39. Let $\mu \vdash n$ such that $\mu^{\prime}=\alpha^{\prime}+e \lambda^{\prime}$ and suppose $D_{F}(1, \alpha) \cong$ $D_{\text {reg }}(|\alpha|)$ and suppose $\lambda \vdash r$. Denote by the largest integer smaller or equal to $n / e-r$ and by $d$ the number of composition factors $D_{F}\left(1,1^{e}\right)$ in $P_{S t}\left(\mathrm{Gl}_{e}(q)\right)$. The number of times, that $D_{F}(1, \mu)$ is a composition factor in the $(\delta k+t)^{\text {th }}$ radical layer of $P_{S t}(G)$ is equal to:

$$
\sum_{\rho \vdash r} \operatorname{comp}(k, d, \rho) \cdot \operatorname{dim}_{F} \operatorname{Hom}_{F S_{r}}\left(S^{\lambda}, M^{\rho} \otimes S^{\left(1^{r}\right)}\right)
$$

where $\delta=2$ if $d=2$ and $\delta=1$ if $d>2$.

\section{Conclusions}

In this section, as promised, we will take a look at other blocks. We will also outline some possible areas of further research or application. 
If we look back, we actually used very seldom that we worked in the unipotent block. One occasion was that the PIMs are self-dual. This is not the case in general. However, the union of blocks we look at are given by semisimple $\ell$-regular elements $s \in G$. Now suppose $s$ is conjugate to $s_{1}^{k_{1}} \times s_{2}^{k_{2}} \times \ldots s_{N}^{k_{N}}$ with $d_{i}=\operatorname{deg} s_{i}$ and $d_{1} k_{1}+\cdots+d_{N} k_{N}=n$. Then we know by the work of Brundan, Dipper and Kleshchev (see [BrDiKl], Thm 2.4e) that this union is Morita equivalent to the block of $\mathrm{Gl}_{d_{1} k_{1}}(q) \times \cdots \times \mathrm{Gl}_{d_{N} k_{N}}(q)$ defined by the same $s$. So we only need to look at blocks defined by $s$ where $s$ is conjugate to $s_{1}^{k_{1}}$. Here the dual module of $D_{F}(s, \lambda)$ is simply $D_{F}\left(s^{-1}, \lambda\right)$. Since there is no structural difference between $s$ and $s^{-1}$ all the results still hold. Another occasion is the description of the cyclic defect group by the condition $e l>n$. This has to be reworked carefully but can be overcome. Last but not least Bonnafé and Rouquier in [BoRou] imply the Morita equivalence with their general result, so we know that the structure of the regular PIM in any block from the structure of the Steinberg PIM.

If we look at the data we actually need to start the combinatorial machinery, we see only two things. The number of times $e$ fits into $n$ and the length of the Steinberg-PIM of $\mathrm{Gl}_{e}(q)$. All the other data, especially the concrete value of $n$ and $e$ is only needed to label the composition factors correctly. For the structure, they are irrelevant. A few calculations for small $n / e$ and small $d$ are given in appendix $\mathrm{C}$

There are a lot of ways to expand the results. The first naturally would be to get rid of the Abelian defect condition. An other direction could be to generalise some of this to other classical groups. A possible application could be in the construction and understanding of a projective generator of the unipotent block, similar to that in the case $e=1$ and the calculation of its endomorphism ring which could lead to the Loewy series for all PIMs. 


\section{A German Summary}

In der modularen Darstellungstheorie der generellen linearen Gruppe in nichtbeschreibender Charakteristik ist bereits Vieles bekannt. Durch die Arbeiten von Dipper und James kennen wir eine Konstruktion und eine Indizierung der irreduziblen Moduln (vgl. [Di1],[Di2],[Ja2],[DiJa1]) und die Berechnung der Zerlegungsmatrizen wurde von den selben Autoren auf die Kenntnis der Zerlegungsmatrizen von $q$-Schur-Algebren zurückgeführt (vgl. [DiJa2]). Weiterhin kennen wir die Verteilung der irreduziblen Moduln auf Harish-Chandra Serien [Hi] und auch die Verteilung auf die Blöcke [FoSri2].

Diese Dissertation beschäftigt sich nun mit einem Teilgebiet der Darstellungstheorie von $\mathrm{Gl}_{n}(q)$, welches größtenteils noch unerforscht ist. Die projektivunzerlegbaren Moduln (im folgenden kurz PIM) und die Berechnung ihrer Struktur stellen eine wichtigen Zwischenschritt zum Verständnis aller unzerlegbaren Moduln dar. Aus allgemeinen Sätzen wissen wir, dass es zu jedem irreduziblen Modul $D$ genau einen PIM $P$ gibt, so dass $D$ der Kopf und der Sockel von $P$ ist. Kenntnis der Zerlegungsmatrix gibt uns außerdem die Kompositionsfaktoren jedes PIMs. In dieser Arbeit soll nun die Frage nach der inneren Struktur, d.h. den Loewyreihen, dieser PIMs gestellt und in Spezialfällen auch beantwortet werden.

Im sehr speziellen Fall eines PIMs, der in einem Block mit zyklischer Defektgruppe liegt, liefern die Arbeiten von Jennings [Je] und Fong und Srinivasan [FoSri1] eine vollständige Antwort. Wir betrachten nun eine Verallgemeinerung davon, den Fall eines Blocks mit abelscher Defektgruppe. Leider können wir die Struktur aller PIMs nur in einem Spezialfall bestimmen. Aber wir bestimmen die Struktur einschließlich der Angabe elementarer Formeln für die PIMs welche im GelfandGraev-Modul enthalten sind.

Um die Notation zu vereinfachen, beschränken wir uns vorerst sogar noch weiter, nämlich auf den unipotenten Block und den darin enthalten PIM aus dem Gelfand-Graev-Modul, welche wir im folgenden als Steinberg-PIM bezeichnen.

Das erste Kapitel etabliert wichtige Notationen. $G$ bezeichne im folgenden die generelle lineare Gruppe der invertierbaren $n \times n$-Matrizen mit Einträgen aus dem endlichen Körper $\operatorname{GF}(q)$. Es sei $F$ ein Körper der Charakteristik $\ell>0$, wobei $\ell$ kein Teiler von $q$ ist. Eine standard Leviuntergruppe $L \leq G$ ist eine Gruppe von Blockdiagonalmatrizen der Form $\mathrm{Gl}_{n_{1}}(q) \times \cdots \times \mathrm{Gl}_{n_{k}}(q)$ mit $n_{1}+\cdots+n_{k}=n$. Außerdem bezeichnen wir mit $e$ die Ordnung von $q$ modulo $\ell$.

Im zweiten Kaptitel tragen wir die für den Fortgang wichtigen Ergebnisse der Darstellungstheorie der $\mathrm{Gl}_{n}(q)$ zusammen. So werden die Funktoren Harish-Chandra Induktion $\left(\mathrm{R}_{L}^{G}\right)$ und Harish-Chandra Restriktion $\left(\mathrm{T}_{L}^{G}\right)$ definiert, die cuspidalen und irreduziblen Moduln konstruiert und die Einteilung der Irreduziblen in Blöcke

und Harish-Chandra Serien angegeben. Zuletzt wiederholen wir noch die Definition des Gelfand-Graev Moduls $\Gamma_{G}$ und definieren den Steinberg-PIM $P_{S t}(G)$ als 
den PIM, der Gelfand-Graev und dem unipotenten Block gemeinsam ist. Dessen wichtigste, im folgenden oft benötigte Eigenschaft ist

$$
\mathrm{T}_{L}^{G} P_{S t}(G) \cong P_{S t}(L):=P_{S t}\left(\mathrm{Gl}_{n_{1}}(q)\right) \otimes \cdots \otimes P_{S t}\left(\mathrm{Gl}_{n_{k}}(q)\right)
$$

wobei die Leviuntergruppe $L=\mathrm{Gl}_{n_{1}}(q) \times \cdots \times \mathrm{Gl}_{n_{k}}(q)$ ist.

Im dritten Kapitel geht es darum, welchen Effekt Harish-Chandra Restriktion auf die Kompositionsfaktoren des Steinberg-PIM hat. Die Basis dafür ist das folgende Korollar basierend auf einem Ergebnis von Dipper und Gruber [DiGr]:

Korollar 1 (Korollar 3.3). Sei e $>1$. Dann hat der Steinberg-PIM $P_{S t}(G)$ genau einen Kompositionsfaktor $D_{F}(1, \lambda)$ mit $\lambda \vdash n$ e-regulär und dieser hat Multiplizität 1. Er wird mit $D_{\text {reg }}(n)$ bezeichnet.

Damit bestimmen wir zunächst die möglichen Konstituenten:

Satz 2 (Theorem 3.5). Sei $\mu \vdash n$ eine Partition von $n$ mit $\mu^{\prime}=\alpha^{\prime}+e \rho^{\prime}$, wobei $\alpha \vdash j$ e-regulär und $\rho \vdash w$. Wenn nun $D_{F}(1, \mu)$ ein Kompositionsfaktor im Steinberg-PIM $P_{S t}(G)$ ist, dann ist $D_{F}(1, \alpha) \cong D_{\text {reg }}(j)$ und $D_{F}\left(1,\left(e \rho^{\prime}\right)^{\prime}\right)$ ist ein Kompositionsfaktor von $P_{S t}\left(\mathrm{Gl}_{\text {ew }}(q)\right)$.

Die Umkehrung ist auch richtig, ihr Beweis benötigt allerdings den folgenden wichtigsten Satz dieses Kapitels, welcher sowohl für die weitere Arbeit zentral als auch für sich selbst interessant ist.

Satz 3 (Theorem 3.7). Sei $D_{F}(1, \mu)$ ein Kompositionsfaktor des Steinberg-PIM $P_{S t}(G)$. Für jede Leviuntergruppe $L \leq G$ gilt, dass $\mathrm{T}_{L}^{G} D_{F}(1, \mu)$ entweder gleich 0 oder direkte Summe von einfachen FL-Moduln ist.

Im vierten Kapitel sind wir dann beim Kern der Dissertation. Zunächst wird die Untermodulstruktur im Fall einer zyklischen Defektgruppe nach Peacock [Pea] zitiert (Theorem 4.4). Damit bestimmen wir die Radikalstruktur von $P_{S t}(L)$ und $\operatorname{End}_{F L}\left(P_{S t}(L)\right)$ wobei wir die Leviuntergruppe $L$ als $L=\mathrm{Gl}_{e}(q)^{r} \times \mathrm{Gl}_{1}(q)^{s}$ wählen und damit $P_{S t}(L)$ aus dem zyklischen Defektfall hervorgeht. Weiter untersuchen wir dann den Endomorphismenring $\operatorname{End}_{F G}\left(\mathrm{R}_{L}^{G} P_{S t}(L)\right)$. Darin finden wir eine Unteralgebra $H$, welche isomorph ist zur Heckealgebra $\mathcal{H}_{1, E}\left(S_{r}\right) \otimes \mathcal{H}_{1, E}\left(S_{s}\right)$ wobei $E=\operatorname{End}_{F L}\left(P_{S t}(L)\right)$. Sie hat die folgende innere Struktur:

Satz 4 (Theorem 4.24). Seien $\left(\lambda_{1}, \lambda_{2}\right)$ und $\left(\mu_{1}, \mu_{2}\right)$ Multipartitionen von $(r, s)$. Die folgende Formel gibt die Anzahl der Kompositionsfaktoren vom Typ $S^{\lambda_{1}} \otimes S^{\lambda_{2}}$ in der $k$-ten Schicht der Radikalreihe des PIMs vom Typ $\left(\mu_{1}, \mu_{2}\right)$ an, wobei die Schichten von oben nach unten, beginnend bei 0 , gezählt werden.

$$
\sum_{\rho \vdash r} \operatorname{comp}(k, d, \rho) \cdot \operatorname{dim}_{F} \operatorname{Hom}_{F S_{r} \otimes F S_{s}}\left(S^{\lambda_{1}} \otimes S^{\lambda_{2}},\left(S^{\mu_{1}} \otimes S^{\mu_{2}}\right) \otimes M^{(\rho,(s))}\right)
$$


Hierbei ist d die Länge der Loewyreihe vom $P_{S t}(L)$ und $\operatorname{comp}(k, d, \rho)$ bezeichnet die Anzahl der Kompositionen von $k$ der Bauart $\left(x_{1}^{\rho_{1}}, \ldots, x_{t}^{\rho_{t}}\right)$ wobei $0 \leq x_{i}<d$ paarweise verschieden sind.

Diese ganzen Überlegungen erledigen im wesentlichen den Spezialfall $e=1$, der dann als nächstes kurz behandelt wird.

Nun folgt ein weiteres Hauptergebnis der Dissertation, welche das Verhalten der Radikalreihe unter Harish-Chandra Restriktion behandelt.

Satz 5 (Theorem 4.28). Sei $L=\mathrm{Gl}_{e}(q)^{r} \times \mathrm{Gl}_{1}(q)^{s}$ mit $s<e$ und sei $e>1$. Dann gilt für jede standard Leviuntergruppe $L \leq M \leq G$ :

$$
\mathrm{T}_{M}^{G} \operatorname{rad}^{i}\left(P_{S t}(G)\right)=\operatorname{rad}^{i}\left(P_{S t}(M)\right)
$$

Der Beweis dieses Satzes beruht auf den folgenden Ideen. Zum einen gilt für $X \leq P_{S t}(G)$, dass $\operatorname{rad}^{i}\left(\mathrm{~T}_{M}^{G} X\right) \subseteq \mathrm{T}_{M}^{G} \operatorname{rad}^{i}(X)$, weil $\mathrm{T}_{M}^{G} \mathrm{hd}(X)$ halbeinfach ist. Damit kann der Gleichheit durch zählen der Kompositionsfaktoren im Kopf jeden Radikals induktiv beigekommen werden. Den Erfolg verdankt diese Zählmethode vor allem der Kenntnis der jeweiligen Lage des Faktors $D_{\text {reg }}$.

Lemma 6 (Lemma 4.30). Mit obigen Bezeichnungen liegt der Kompositionsfaktor $D_{\text {reg }}(n)$ von $P_{S t}(G)$ im Kopf von $\operatorname{rad}^{r}\left(P_{S t}(G)\right)$.

Nachdem nun die Struktur von $P_{S t}(L)$ aus dem zyklischen Fall abgeleitet wurde und Harish-Chandra Restriktion die Radikalstruktur erhält fehlt zur Berechnung der Radikalstruktur von $P_{S t}(G)$ nur noch die Information, wie sich einzelne Kompositionsfaktoren unter Harish-Chandra Restriktion verhalten. Da die Abbildung leider nicht injektiv ist, also Informationen verloren gehen benutzen wir insbesondere die bekannte Struktur von $H$, um zu folgendem Endergebnis zu kommen:

Satz 7 (Theorem 4.39). Sei $\mu \vdash n$ derart, dass $\mu^{\prime}=\alpha^{\prime}+e \lambda^{\prime}$, wobei $D_{F}(1, \alpha) \cong D_{\text {reg }}(|\alpha|)$ und $\lambda \vdash r$. Sei weiter $t=[n / e-r]$ und d die Anzahl der Kompositionsfaktoren der Form $D_{F}\left(1,1^{e}\right)$ in $P_{S t}\left(\mathrm{Gl}_{e}(q)\right)$. Dann ist die Anzahl der Kompositionsfaktoren vom Typ $D_{F}(1, \mu)$ in der $(\delta k+t)$-ten Radikalschicht gleich dem folgenden Ausdruck:

$$
\sum_{\rho \vdash r} \operatorname{comp}(k, d, \rho) \cdot \operatorname{dim}_{F} \operatorname{Hom}_{F S_{r}}\left(S^{\lambda}, M^{\rho} \otimes S^{\left(1^{r}\right)}\right)
$$

Dabei ist $\delta=\left\{\begin{array}{ll}2 & \text { falls } d=2 \\ 1 & \text { sonst }\end{array}\right.$.

Wir beenden die Arbeit mit einem Ausblick auf alle PIMs des Gelfand-Graev und mögliche Verallgemeinerungen. Ein Anhang mit der Definition der MullineuxAbbildung und konkreten Beispielen schließt das Ganze ab. 


\section{B The Mullineux Map}

For the calculation of $D_{\text {reg }}(n)$ we need an explicit description of the Mullineux map. We will give this description here. We will not prove that this purely combinatorial map coincides with the result in representation theory we really claim. That was done by Brundan in [Bru]. The map itself is due to Mullineux $[\mathrm{Mu}]$.

The Young diagram of a partition $\lambda=\left(\lambda_{1}, \lambda_{2}, \ldots, \lambda_{r}\right)$ is the following set of points in the $x-y$ plane:

$$
\left\{(i, j) \mid 1 \leq j \leq \lambda_{i}, 1 \leq i \leq r\right\}
$$

The $y$-axis is taken as pointing downwards so the row 1 is at the top. The e-edge of a partition consists of $e$-segments all but at most one of which contain $e$ points. The first $e$-segment comprises either the first $e$ points of the rim (starting with the longest row) or the entire rim if its length is less than $e$. The next $e$-segment is obtained similarly starting in the row next below that contained the end of the previous $e$-segment. This process continues until the final row is reached.

Let $\lambda=\lambda^{(0)}, \lambda^{(1)}, \ldots, \lambda^{(\alpha)}=\emptyset$ be the partitions we get by removing the respective $e$-edges. Denote by $a_{i}$ the number of points of the respective $e$-edges and by $r_{i}$ the number of rows of $\lambda^{(i)}$.

The datum $\left(\begin{array}{llll}a_{0} & a_{1} & \ldots & a_{\alpha-1} \\ r_{0} & r_{1} & \ldots & r_{\alpha-1}\end{array}\right)$ determines $\lambda$.

Now we define $s_{i}:=a_{i}-r_{1}+\epsilon_{i}$ where $\epsilon_{i}=0$ if $e$ divides $a_{i}$ and $\epsilon_{i}=1$ otherwise. Then $\left(\begin{array}{cccc}a_{0} & a_{1} & \ldots & a_{\alpha-1} \\ s_{0} & s_{1} & \ldots & s_{\alpha-1}\end{array}\right)$ defines an $e$-regular partition $m(\lambda)$ and we have $D_{\text {reg }}(n)=D_{F}(1, m(n))$.

We give an example for $e=4$ and get the following table: 


\begin{tabular}{|c|c|c|c|c|c|}
\hline$\lambda$ & $\left(\begin{array}{lll}a_{0} & a_{1} & \ldots \\
r_{0} & r_{1} & \ldots\end{array}\right.$ & $\left.\begin{array}{l}a_{\alpha-1} \\
r_{\alpha-1}\end{array}\right)$ & $\left(\begin{array}{lll}a_{0} & a_{1} & \ldots \\
s_{0} & s_{1} & \ldots\end{array}\right.$ & $\left.\begin{array}{l}a_{\alpha-1} \\
s_{\alpha-1}\end{array}\right)$ & $m(\lambda)$ \\
\hline (1) & $\left(\begin{array}{l}1 \\
1\end{array}\right)$ & & $\left(\begin{array}{l}1 \\
1\end{array}\right)$ & & (1) \\
\hline (2) & $\left(\begin{array}{l}2 \\
1\end{array}\right)$ & & $\left(\begin{array}{l}2 \\
2\end{array}\right)$ & & $\left(1^{2}\right)$ \\
\hline (3) & & & $\left(\begin{array}{l}3 \\
3\end{array}\right)$ & & $\left(1^{3}\right)$ \\
\hline (4) & & & $\left(\begin{array}{l}4 \\
3\end{array}\right)$ & & $\left(21^{2}\right)$ \\
\hline (5) & $\left(\begin{array}{ll}4 & 1 \\
1 & 1\end{array}\right)$ & & $\left(\begin{array}{ll}4 & 1 \\
3 & 1\end{array}\right)$ & & $\left(2^{2} 1\right)$ \\
\hline (6) & $\left(\begin{array}{ll}4 & 2 \\
1 & 1\end{array}\right)$ & & $\left(\begin{array}{ll}4 & 2 \\
3 & 2\end{array}\right)$ & & $\left(2^{3}\right)$ \\
\hline (7) & $\left(\begin{array}{ll}4 & 3 \\
1 & 1\end{array}\right)$ & & $\left(\begin{array}{ll}4 & 3 \\
3 & 3\end{array}\right)$ & & $\left(32^{2}\right)$ \\
\hline (8) & $\left(\begin{array}{ll}4 & 4 \\
1 & 1\end{array}\right)$ & & $\left(\begin{array}{ll}4 & 4 \\
3 & 3\end{array}\right)$ & & $\left(3^{2} 2\right)$ \\
\hline
\end{tabular}

\section{Examples}

In the spirit of the remark in the last chapter we will only give structural examples, depending on the index $w$ of $e$ in $n$ and the number of composition factors in $P_{S t}\left(\mathrm{Gl}_{e}(q)\right)$ henceforth denoted by $d+1$. So we need a new notation for our generic composition factors. We write $(x, \rho)$ to denote the composition factor $\mathrm{R}_{L}^{G} D_{r e g}(x e+s) \otimes D_{F}\left(1,\left(e \rho^{\prime}\right)^{\prime}\right)$ with $n=e(x+|\rho|)+s$ with $s<e$ and $L=$ $\mathrm{Gl}_{x e+s}(q) \times \mathrm{Gl}_{e|\rho|}(q)$.

We start with the case of cyclic defect, that is $w=1$.

\begin{tabular}{|c|cc|}
\hline \multicolumn{3}{|c|}{$w=1, d=2$} \\
\hline layer & $(0,(1))$ & $(1,())$ \\
\hline 0 & 1 & 0 \\
1 & 0 & 1 \\
2 & 1 & 0 \\
\hline
\end{tabular}

\begin{tabular}{|c|cc|}
\hline \multicolumn{3}{|c|}{$w=1, d>2$} \\
\hline layer & $(0,(1))$ & $(1,())$ \\
\hline 0 & 1 & 0 \\
1 & 1 & 1 \\
2 & 1 & 0 \\
$\vdots$ & $\vdots$ & $\vdots$ \\
$d-1$ & 1 & 0 \\
\hline
\end{tabular}

The next step $w=2$ is already more complicated and a general model cannot be 
given.

\begin{tabular}{|c|cccc|}
\hline \multicolumn{4}{|l|}{$w=2, d=2$} \\
\hline layer & $\left(0,\left(1^{2}\right)\right)$ & $(0,(2))$ & $(1,(1))$ & $(2,())$ \\
\hline 0 & 1 & 0 & 0 & 0 \\
1 & 0 & 0 & 1 & 0 \\
2 & 1 & 1 & 0 & 1 \\
3 & 0 & 0 & 1 & 0 \\
4 & 1 & 0 & 0 & 0 \\
\hline
\end{tabular}

\begin{tabular}{|c|cccc|}
\hline \multicolumn{6}{|c|}{$w=2, d=3$} \\
\hline layer & $\left(0,\left(1^{2}\right)\right)$ & $(0,(2))$ & $(1,(1))$ & $(2,())$ \\
\hline 0 & 1 & 0 & 0 & 0 \\
1 & 1 & 1 & 1 & 0 \\
2 & 2 & 1 & 1 & 1 \\
3 & 1 & 1 & 1 & 0 \\
4 & 1 & 0 & 0 & 0 \\
\hline
\end{tabular}

\begin{tabular}{|c|cccc|}
\hline \multicolumn{4}{|l|}{$w=2, d=4$} \\
\hline layer & $\left(0,\left(1^{2}\right)\right)$ & $(0,(2))$ & $(1,(1))$ & $(2,())$ \\
\hline 0 & 1 & 0 & 0 & 0 \\
1 & 1 & 1 & 1 & 0 \\
2 & 2 & 1 & 1 & 1 \\
3 & 2 & 2 & 1 & 0 \\
4 & 2 & 1 & 0 & 0 \\
5 & 1 & 1 & 0 & 0 \\
6 & 1 & 0 & 0 & 0 \\
\hline
\end{tabular}

\begin{tabular}{|c|cccc|}
\hline \multicolumn{4}{|c|}{$w=2, d=5$} & \\
& & \\
\hline layer & $\left(0,\left(1^{2}\right)\right)$ & $(0,(2))$ & $(1,(1))$ & $(2,())$ \\
\hline 0 & 1 & 0 & 0 & 0 \\
1 & 1 & 1 & 1 & 0 \\
2 & 2 & 1 & 1 & 1 \\
3 & 2 & 2 & 1 & 0 \\
4 & 3 & 2 & 0 & 0 \\
5 & 2 & 2 & 0 & 0 \\
6 & 2 & 1 & 0 & 0 \\
7 & 1 & 1 & 0 & 0 \\
8 & 1 & 0 & 0 & 0 \\
\hline
\end{tabular}


Even more complicated gets $w=3$ :

\begin{tabular}{|c|ccccccc|}
\hline \multicolumn{2}{|l}{$w=3, d=2$} & \multicolumn{10}{|c|}{} \\
\hline layer & $\left(0,\left(1^{3}\right)\right)$ & $(0,(21))$ & $(0,(3))$ & $\left(1,\left(1^{2}\right)\right)$ & $(1,(2))$ & $(2,(1))$ & $(3,())$ \\
\hline 0 & 1 & 0 & 0 & 0 & 0 & 0 & 0 \\
1 & 0 & 0 & 0 & 1 & 0 & 0 & 0 \\
2 & 1 & 1 & 0 & 0 & 0 & 1 & 0 \\
3 & 0 & 0 & 0 & 1 & 1 & 0 & 1 \\
4 & 1 & 1 & 0 & 0 & 0 & 1 & 0 \\
5 & 0 & 0 & 0 & 1 & 0 & 0 & 0 \\
6 & 1 & 0 & 0 & 0 & 0 & 0 & 0 \\
\hline
\end{tabular}

\begin{tabular}{|c|ccccccc|}
\hline \multicolumn{2}{|l}{$w=3, d=3$} & \multicolumn{1}{|c|}{$\mid$} \\
\hline layer & $\left(0,\left(1^{3}\right)\right)$ & $(0,(21))$ & $(0,(3))$ & $\left(1,\left(1^{2}\right)\right)$ & $(1,(2))$ & $(2,(1))$ & $(3,())$ \\
\hline 0 & 1 & 0 & 0 & 0 & 0 & 0 & 0 \\
1 & 1 & 1 & 0 & 1 & 0 & 0 & 0 \\
2 & 2 & 2 & 0 & 1 & 1 & 1 & 0 \\
3 & 2 & 2 & 1 & 2 & 1 & 1 & 1 \\
4 & 2 & 2 & 0 & 1 & 1 & 1 & 0 \\
5 & 1 & 1 & 0 & 1 & 0 & 0 & 0 \\
6 & 1 & 0 & 0 & 0 & 0 & 0 & 0 \\
\hline
\end{tabular}

\begin{tabular}{|c|cccccccc|}
\hline \multicolumn{2}{|l}{$w=3, d=4$} \\
\hline layer & $\left(0,\left(1^{3}\right)\right)$ & $(0,(21))$ & $(0,(3))$ & $\left(1,\left(1^{2}\right)\right)$ & $(1,(2))$ & $(2,(1))$ & $(3,())$ \\
\hline 0 & 1 & 0 & 0 & 0 & 0 & 0 & 0 \\
1 & 1 & 1 & 0 & 1 & 0 & 0 & 0 \\
2 & 2 & 2 & 0 & 1 & 1 & 1 & 0 \\
3 & 3 & 3 & 1 & 2 & 1 & 1 & 1 \\
4 & 3 & 4 & 1 & 2 & 2 & 1 & 0 \\
5 & 3 & 4 & 1 & 2 & 1 & 0 & 0 \\
6 & 3 & 3 & 1 & 1 & 1 & 0 & 0 \\
7 & 2 & 2 & 0 & 1 & 0 & 0 & 0 \\
8 & 1 & 1 & 0 & 0 & 0 & 0 & 0 \\
9 & 1 & 0 & 0 & 0 & 0 & 0 & 0 \\
\hline
\end{tabular}




\begin{tabular}{|c|ccccccc|}
\hline \multicolumn{2}{|l}{$w=3, d=5$} \\
\hline layer & $\left(0,\left(1^{3}\right)\right)$ & $(0,(21))$ & $(0,(3))$ & $\left(1,\left(1^{2}\right)\right)$ & $(1,(2))$ & $(2,(1))$ & $(3,())$ \\
\hline 0 & 1 & 0 & 0 & 0 & 0 & 0 & 0 \\
1 & 1 & 1 & 0 & 1 & 0 & 0 & 0 \\
2 & 2 & 2 & 0 & 1 & 1 & 1 & 0 \\
3 & 3 & 3 & 1 & 2 & 1 & 1 & 1 \\
4 & 4 & 5 & 1 & 2 & 2 & 1 & 0 \\
5 & 4 & 6 & 2 & 3 & 2 & 0 & 0 \\
6 & 5 & 6 & 2 & 2 & 2 & 0 & 0 \\
7 & 4 & 6 & 2 & 2 & 1 & 0 & 0 \\
8 & 4 & 5 & 1 & 1 & 1 & 0 & 0 \\
9 & 3 & 3 & 1 & 1 & 0 & 0 & 0 \\
10 & 2 & 2 & 0 & 0 & 0 & 0 & 0 \\
11 & 1 & 1 & 0 & 0 & 0 & 0 & 0 \\
12 & 1 & 0 & 0 & 0 & 0 & 0 & 0 \\
\hline
\end{tabular}




\section{References}

[Ack] B. Ackermann, A short note on Howlett-Lehrer Theory, Arch. Math. 79, p. 161-166, 2002

[BoRou] C. Bonnafé, R. Rouquier, Catégories dériv'ees et variétés de DeligneLusztig, Publications de Mathématique de l'IHÉS 97, p. 1-59, 2003

[BrMi] M. Broué, J. Michel, Blocs et séries des Lusztig dans un groupe reductif finis, J. reine angew. Math. 395, p. 56-67, 1989

[Bru] J. Brundan, Modular branching rules and the Mullineux map for Hecke algebras of type $A$, Proc. London Math. Soc. (3) 77, p. 551-581, 1998

[BrDiKl] J. Brundan, R. Dipper, A. Kleshchev, Quantum Linear Groups and Representations of $\mathrm{Gl}_{n}(q)$, AMS Memoirs 706, 2001

[Carter] R.Carter, Finite Groups of Lie Type, Wiley 1985

[CuRe1] Curtis, Reiner, Methods of Representation Theory I, Wiley 1981

[Di1] R. Dipper, On decomposition numbers of the finite general linear group, Trans. AMS 290, p. 315-344, 1985

[Di2] R. Dipper, On decomposition numbers of the finite general linear group II, Trans. AMS 292, p.123-133, 1985

[Di3] R. Dipper, On Quotients of Hom-Functors and Representations of General Linear Groups II, J. Algebra 209, p. 199-269, 1998

[DiDu] R. Dipper, J. Du, Harish-Chandra vertices, J. reine u. angew. Math 437, p. 101-130, 1993

[DiFl] R. Dipper, P. Fleischmann, Modular Harish-Chandra theory I, Math. Z. 211, p. 49-72, 1992

[DiGr] R. Dipper, J. Gruber, General $q$-Schur algebras and modular representation theory of finite groups with split $(B, N)$-pairs, J. reine angew. Math. 511, p. 145-191, 1999

[DiJa1] R. Dipper, G. James, Identification of the irreducible modular representations of $\mathrm{Gl}_{n}(q)$, J. Algebra 104, p. 266-288, 1986

[DiJa2] R. Dipper, G. James, The q-Schur Algebra, Proc. LMS (3) 59, p. 23-50, 1989

[FoSri1] P. Fong, B. Srinivasan, Blocks with cyclic defect groups in $G L(n, q)$, Bull. AMS 3, p 1041-1044, 1980 
[FoSri2] P. Fong, B. Srinivasan, The blocks of the finite general linear and unitary groups, Invent. Math. 69, p. 109-153, 1982

[Gel] S.I. Gelfand, Representations of the full linear group over a finite field, Math. USSR-Sb 12, 1970

[GelGr] I. Gelfand, M. Graev, Construction of irreducible representations of simple algebraic groups over a finite field, Doklady Akad. Nauk SSSR 147, p. $529-532,1962$

[Hi] G. Hiss, Harish-Chandra series of Brauer characters in a finite group with a split $B, N$-pair, J. LMS (2) 48, p. 219-228, 1993

[HoLe1] R. Howlett, G. Lehrer, Induced cuspidal representations and generalized Hecke rings, Invent. Math 58, p37-64, 1980

[HoLe2] R. Howlett, G. Lehrer, On Harish-Chandra induction for modules of Levi subgroups, J. of Algebra 165, p. 172-183, 1994

[Hu] B. Huppert, Endliche Gruppen I, Springer 1967

[Ja1] G. James, The Representation Theory of the Symmetric Groups, Springer Lecture Notes 682, 1978

[Ja2] G. James, Representations of General Linear Groups, LMS Lecture Note Series 94, Cambeidge Univ. Press, 1984

[Ja3] G. James, The irreducible representations of the finite general linear groups, Proc. LMS (3) 52, p. 236-268, 1986

[Ja4] G. James, The Decomposition Matrices of $\mathrm{Gl}_{n}(q)$ for $n \leq 10$, Proc. LMS (3) 60, p. 225-265, 1990

[Je] S. Jennings, The structure of the group ring of a $p$-group over a modular field, Trans. AMS 50, p. 175-185, 1941

$[\mathrm{Mu}] \quad$ G. Mullineux, Bijections of $p$-regular partitions and $p$-modular irreducibles of the symmetric group, J. LMS (2) 20, p. 60-66, 1979

[Pea] R. Peacock, Blocks with cyclic defect group, J. Algebra 34, p. 232-259, 1975

[Weir] A. Weir, Sylow $p$-subgroups of the classical groups over finite fields with characteristic prime to $p$. Proc. AMS 6, p. 529-533, 1955 